\title{
ON THE RESIDUAL SPECTRUM OF HERMITIAN QUATERNIONIC INNER FORM OF $\mathrm{SO}_{8}$
}

\author{
Neven Grbac \\ University of Rijeka, Croatia
}

\begin{abstract}
In this paper we decompose the residual spectrum supported in the minimal parabolic subgroup of an inner form of the split group $\mathrm{SO}_{8}$. The approach is the Langlands spectral theory. However, since the group is non-quasi-split, it is out of the scope of the Langlands-Shahidi method and the new technique for the normalization of standard intertwining operators is developed. The decomposition shows interesting parts of the residual spectrum not appearing in the case of quasi-split groups.
\end{abstract}

\section{INTRODUCTION}

This paper deals with the residual spectrum of the hermitian quaternionic group which is an inner form of the split group $\mathrm{SO}_{8}$. Here the part of the residual spectrum coming from the minimal parabolic subgroup is obtained. After the early attempts of Jacquet in [19] and the appendix of Langlands in [30], the first time the residual spectrum was classified in a serious way was by Mœglin and Waldspurger in [37]. The residual spectrum of quasi-split groups has been considered afterwards by several authors such as Moeglin [33, 34, 35], Kim [22, 23, 26], Žampera [55], Kon-No [27]. Their approach is based on the Langlands spectral theory $([30,38])$ and uses the Langlands-Shahidi method $([43,45])$ for the normalization of intertwining operators.

However, our situation is different. This paper is dealing with non-quasisplit groups and we had to develop a new technique for normalization in order to overcome the difficulty of not having the Langlands-Shahidi method at disposal. Moreover, when compared to split group $\mathrm{SO}_{8}$, the results of this paper (see for example Theorem 2.31 and Theorem 2.37) show some

2000 Mathematics Subject Classification. 11F70, 22E55.

Key words and phrases. Automorphic forms, spectral decomposition, residual spectrum, inner forms. 
interesting parts of the residual spectrum not appearing in the case of split groups. The reason for that lies in different local normalization factors at split and non-quasi-split places.

The method was already applied by the author to obtain the residual spectra of inner forms of $\mathrm{SO}_{4}$ and $\mathrm{Sp}_{4}$ in [11], an inner form of $\mathrm{Sp}_{8}$ in [13], and some simple parts of the residual spectra of inner forms of $\mathrm{SO}_{4 n}$ and $\mathrm{Sp}_{4 n}$ in general in [12]. Also, the residual spectrum of the general linear group over a division algebra was obtained in [14], but the calculation relies on the results in the body of the paper which were obtained using the comparison of trace formulas.

Besides the Langlands spectral theory, another approach to the discrete spectrum is through the trace formula. As explained in [2, 3, 4], Arthur has developed the trace formula in order to describe the discrete spectrum of connected split classical groups. His description is still conjectural since it depends on the fundamental lemma. Furthermore, the problem of distinguishing the residual inside the discrete spectrum still remains. In [36] Mœglin has done the first step in that direction. However, since our inner form is non-quasi-split we do not follow Arthur's approach in this paper.

Before giving a brief description of the method and results we introduce some notation. Let $k$ be an algebraic number field and $\mathbb{A}$ the ring of adeles of $k$. For every place $v$ of $k$ we denote by $k_{v}$ the completion of $k$ at $v$. Let $D$ be a quaternion algebra central over $k$ and $\tau$ the usual involution fixing the center of $D$. Then $D$ splits at all but finitely many places $v$ of $k$ i.e. at those places the completion $D \otimes_{k} k_{v}$ is isomorphic to the additive group $M\left(2, k_{v}\right)$ of $2 \times 2$-matrices with coefficients in $k_{v}$. At finitely many places $v$ of $k$ where $D$ is non-split the completion $D \otimes_{k} k_{v}$ is isomorphic to the quaternion algebra $D_{v}$ central over $k_{v}$. In this paper we assume that $D$ splits at all archimedean places and the finite set of nonarchimedean places where $D$ is non-split is denoted by $S_{D}$. The cardinality of $S_{D}$, denoted by $\left|S_{D}\right|$, is even for every $D$.

Let us now introduce groups considered in this paper. Denote by $\mathrm{GL}_{n}$, $\mathrm{SL}_{n}$ and $\mathrm{SO}_{2 n}$ split algebraic groups defined over $k$ as usual. Fixing the basis of underlying vector space these are groups of all invertible $n \times n-$ matrices, all unit determinant $n \times n$-matrices and the connected component of the group of isometries of the bilinear form defined by the $2 n \times 2 n$-matrix $J_{2 n}$ having 1 's on the secondary diagonal and zeroes elsewhere.

The group of invertible $n \times n$-matrices with coefficients in $D$ regarded as a reductive algebraic group over $k$ is denoted by $\mathrm{GL}_{n}^{\prime}$. Then $\mathrm{GL}_{n}^{\prime}\left(k_{v}\right) \cong$ $\mathrm{GL}_{2 n}\left(k_{v}\right)$ for every place $v \notin S_{D}$. For a non-split place $v \in S_{D}$ the group $\mathrm{GL}_{n}^{\prime}\left(k_{v}\right)$ is the group of invertible $n \times n$-matrices with coefficients in $D_{v}$. Let $\mathrm{SL}_{n}^{\prime}$ be the subgroup of matrices in $\mathrm{GL}_{n}^{\prime}$ having the reduced norm of the determinant equal to 1 . It is a reductive algebraic group defined over $k$ and $\mathrm{SL}_{n}^{\prime}\left(k_{v}\right) \cong \mathrm{SL}_{2 n}\left(k_{v}\right)$ for every place $v \notin S_{D}$. 
Let $V$ be the $2 n$-dimensional right vector space over $D$. We fix the basis $\left\{e_{1}, \ldots, e_{2 n}\right\}$ of $V$. Then $\left(e_{i}, e_{j}\right)=\delta_{i, 2 n-j+1}$ for $1 \leqslant i \leqslant j \leqslant n$ defines an antihermitian form on $V$ by

$$
\left(v, v^{\prime}\right)=-\tau\left(\left(v^{\prime}, v\right)\right) \quad \text { and } \quad\left(v x, v^{\prime} x^{\prime}\right)=\tau(x)\left(v, v^{\prime}\right) x^{\prime}
$$

for all $v, v^{\prime} \in V$ and $x, x^{\prime} \in D$. The group of isometries of the form ( , ) regarded as a reductive algebraic group defined over $k$ will be denoted by $G_{n}^{\prime}$. It is an inner form of the group $\mathrm{SO}_{4 n}$. Hence $G_{n}^{\prime}\left(k_{v}\right) \cong \mathrm{SO}_{4 n}\left(k_{v}\right)$ for every place $v \notin S_{D}$.

It is the group $G_{2}^{\prime}$ defined above that we consider in this paper. Let $T^{\prime}$ be the maximal split torus in $G_{2}^{\prime}$. It is isomorphic to $\mathrm{GL}_{1} \times \mathrm{GL}_{1}$. The minimal parabolic subgroup $P_{0}^{\prime}=M_{0}^{\prime} N_{0}^{\prime}$ of $G_{2}^{\prime}$ defined over $k$ has the Levi factor $M_{0}^{\prime} \cong \mathrm{GL}_{1}^{\prime} \times \mathrm{GL}_{1}^{\prime}$. The two proper standard parabolic subgroups of $G_{2}^{\prime}$ are $P_{1}^{\prime}=M_{1}^{\prime} N_{1}^{\prime}$ with the Levi factor $M_{1}^{\prime} \cong \mathrm{GL}_{2}^{\prime}$ and $P_{2}^{\prime}=M_{2}^{\prime} N_{2}^{\prime}$ with the Levi factor $M_{2}^{\prime} \cong \mathrm{GL}_{1}^{\prime} \times G_{1}^{\prime}$.

The goal of this paper is to determine the residual spectrum of the group $G_{2}^{\prime}(\mathbb{A})$ supported in the minimal parabolic subgroup. Residual spectrum is the orthogonal complement of the space of cuspidal automorphic forms inside the discrete spectrum. It is the direct sum of irreducible representations which can be described in terms of cuspidal automorphic representations of Levi subgroups using parabolic induction. By the Langlands spectral theory [30], [38] the automorphic representations forming residual spectrum are realized as residues of the Eisenstein series attached to cuspidal automorphic representations of Levi subgroups. The poles of Eisenstein series coincide with the poles of their constant terms which can be expressed as the sum of standard intertwining operators. The poles of intertwining operators are calculated using their normalization by a ratio of L-functions. For quasi-split groups normalization is given by the Langlands-Shahidi method. However, we use the new technique based on the fact that $G_{2}^{\prime}$ is an inner form of $\mathrm{SO}_{8}$ to transfer the normalization by $\mathrm{L}-$ functions from split case of $\mathrm{SO}_{8}$ to the case of non-quasi-split $G_{2}^{\prime}$.

In this paper we describe the part of the residual spectrum of $G_{2}^{\prime}(\mathbb{A})$ coming from the poles of the Eisenstein series attached to cuspidal automorphic representations of the Levi factor $M_{0}^{\prime}(\mathbb{A})$ of the minimal parabolic subgroup of $G_{2}^{\prime}$. In a separate paper [15], we will calculate the Arthur parameters for the residual spectrum of $G_{2}^{\prime}(\mathbb{A})$ along with the Arthur parameters for the residual spectra of inner forms of the split groups $\mathrm{SO}_{4}, \mathrm{Sp}_{4}$ and $\mathrm{Sp}_{8}$.

The paper is divided into two Sections. In Section 1 for every place $v$ of $k$ the normalization of the local intertwining operators using certain ratios of $\mathrm{L}-$ functions is given. This Section is divided into three Subsections where normalization is defined for generic representations at the split place, nongeneric representations at the split place and representations at the non-split place, respectively. It is proved that the normalized intertwining operators are 
holomorphic and non-vanishing in the closure of the positive Weyl chamber (except at the origin in some cases) and on certain line segments required in the calculation outside that closure. Thus the calculation of poles is reduced to the analytic properties of $\mathrm{L}$-functions. In Section 2 the calculation is performed and the decomposition of the residual spectrum of the group $G_{2}^{\prime}(\mathbb{A})$ coming from minimal parabolic subgroup is obtained. It is given in Theorems $2.12,2.17,2.18,2.25,2.26,2.27,2.31,2.35,2.37$ of that Section.

When studying the poles of the Eisenstein series it is enough to consider the real poles. There is no loss in generality because that can be achieved just by twisting a cuspidal automorphic representation of a Levi factor by the appropriate imaginary power of the absolute value of the reduced norm of the determinant. Hence, this assumption is just a convenient choice of coordinates.

We should remark that in this paper the usual parabolic induction from standard parabolic subgroup $P$ of $G$ with the Levi decomposition $P=M N$ will be denoted by $\operatorname{Ind}_{M}^{G}$ instead of $\operatorname{Ind}_{P}^{G}$. This will not cause any confusion since all the parabolic subgroups appearing in the paper are standard.

This paper is a part of the author's Ph.D. Thesis. Other parts are papers $[11,12,13]$. I would like to thank my advisor G. Muic for many useful discussions and constant help during the preparation of this paper. I would like to thank M. Tadić for supporting my research and for his interest in my work. The conversations with H. Kim and E. Lapid were useful in clarifying several issues in automorphic forms and with I. Badulescu in representation theory of $\mathrm{GL}_{n}$ over division algebras. Also I would like to thank my friend M. Hanzer for many useful conversations on the local representation theory of hermitian quaternionic groups. I am grateful to A. Žgaljić for carefully drawing the figures for this paper. And finally, I would like to thank my wife Tiki for being my everlasting inspiration.

\section{NORMALIZATION OF LOCAL INTERTWINING OPERATORS}

In this Section we give the normalization of intertwining operators for $G_{2}^{\prime}\left(k_{v}\right)$. For the local components of a cuspidal automorphic representation $\pi^{\prime} \cong \otimes \pi_{v}^{\prime}$ of the Levi factor $M_{0}^{\prime}(\mathbb{A}) \cong \mathrm{GL}_{1}^{\prime}(\mathbb{A}) \times \mathrm{GL}_{1}^{\prime}(\mathbb{A})$ of $G_{2}^{\prime}(\mathbb{A})$ we prove the holomorphy and non-vanishing of the normalized intertwining operators in the closure of the positive Weyl chamber (except at the origin in some cases) and at certain open intervals outside that closure required in the calculation. This is done for every place $v$ of $k$ separately.

But first we describe the structure of the group $G_{2}^{\prime}$ in more detail. Let $T^{\prime}$ be the maximal split torus in $G_{2}^{\prime}$. It is isomorphic to $\mathrm{GL}_{1} \times \mathrm{GL}_{1}$. Denote by $\Phi^{\prime}$ the set of roots of $G_{2}^{\prime}$ with respect to $T^{\prime}$. Then

$$
\Phi^{\prime}=\left\{ \pm e_{1} \pm e_{2}, \pm 2 e_{1}, \pm 2 e_{2}\right\},
$$


where $e_{i}\left(t_{1}, t_{2}\right)=t_{i}$ for all $\left(t_{1}, t_{2}\right) \in T^{\prime} \cong \mathrm{GL}_{1} \times \mathrm{GL}_{1}$. For the set of positive roots take

$$
\Phi^{\prime+}=\left\{e_{1} \pm e_{2}, 2 e_{1}, 2 e_{2}\right\} .
$$

The corresponding set of simple roots is

$$
\Delta^{\prime}=\left\{e_{1}-e_{2}, 2 e_{2}\right\} .
$$

Let $W$ be the Weyl group of $\left(G_{2}^{\prime}, T^{\prime}\right)$. If $w_{1}$ is the simple reflection with respect to $e_{1}-e_{2}$, and $w_{2}$ with respect to $2 e_{2}$, then

$$
W=\left\{1, w_{1}, w_{2}, w_{1} w_{2}, w_{2} w_{1}, w_{1} w_{2} w_{1}, w_{2} w_{1} w_{2}, w_{1} w_{2} w_{1} w_{2}\right\} .
$$

The minimal parabolic subgroup $P_{0}^{\prime}=M_{0}^{\prime} N_{0}^{\prime}$ of $G_{2}^{\prime}$ defined over $k$ has the Levi factor $M_{0}^{\prime} \cong \mathrm{GL}_{1}^{\prime} \times \mathrm{GL}_{1}^{\prime}$. Then the two proper standard parabolic subgroups of $G_{2}^{\prime}$ are $P_{1}^{\prime}=M_{1}^{\prime} N_{1}^{\prime}$ with the Levi factor $M_{1}^{\prime} \cong \mathrm{GL}_{2}^{\prime}$ corresponding to the simple root $e_{1}-e_{2}$ and $P_{2}^{\prime}=M_{2}^{\prime} N_{2}^{\prime}$ with the Levi factor $M_{2}^{\prime} \cong \mathrm{GL}_{1}^{\prime} \times G_{1}^{\prime}$ corresponding to the simple root $2 e_{2}$. Let $W\left(M_{i}^{\prime}\right)$ denote the quotient group of the normalizer of $M_{i}^{\prime}$ modulo $M_{i}^{\prime}$ viewed as a subgroup of $W$. Observe that $W\left(M_{0}^{\prime}\right)=W$.

Let $P_{i}=M_{i} N_{i}$ be the split form of the parabolic subgroup $P_{i}^{\prime}=M_{i}^{\prime} N_{i}^{\prime}$ for $i=0,1,2$. Then $P_{i}$ is the standard parabolic subgroup of the split $\mathrm{SO}_{8}$ and $M_{0} \cong \mathrm{GL}_{2} \times \mathrm{GL}_{2}, M_{1} \cong \mathrm{GL}_{4}$ and $M_{2} \cong \mathrm{GL}_{2} \times \mathrm{SO}_{4}$. Let $W\left(M_{i}\right)$ denote again the quotient group of the normalizer of $M_{i}^{\prime}$ modulo $M_{i}^{\prime}$ viewed as a subgroup of $W$. Then $W\left(M_{i}\right) \cong W\left(M_{i}^{\prime}\right)$. Throughout this paper we fix matrix representatives of Weyl group elements as in Section 2 of [21].

Since $M_{0}^{\prime} \cong \mathrm{GL}_{1}^{\prime} \times \mathrm{GL}_{1}^{\prime}$, before proceeding we define certain local and global lift of representations from $\mathrm{GL}_{1}^{\prime}$ to $\mathrm{GL}_{2}$. They are given by the Jacquet-Langlands correspondence as in Section 8 of [10]. More precisely, let $\pi^{\prime} \cong \otimes_{v} \pi_{v}^{\prime}$ be a cuspidal automorphic representation of $\mathrm{GL}_{1}^{\prime}(\mathbb{A})$ which is not one-dimensional. Then, at non-split places the lift $\pi_{v}$ of $\pi_{v}^{\prime}$ is the square-integrable representation of $\mathrm{GL}_{2}\left(k_{v}\right)$ defined by a character relation as in Theorem (8.1) of [10]. At split places $\mathrm{GL}_{1}^{\prime}\left(k_{v}\right) \cong \mathrm{GL}_{2}\left(k_{v}\right)$ and the lift is just $\pi_{v} \cong \pi_{v}^{\prime}$. The global lift of $\pi^{\prime}$ is defined using local lifts as $\pi \cong \otimes_{v} \pi_{v}$. By Theorem (8.3) of [10] the global lift $\pi$ is isomorphic to a cuspidal automorphic representation of $\mathrm{GL}_{2}(\mathbb{A})$. Hence, its local components $\pi_{v}$ are generic.

Let $\chi \circ \operatorname{det}^{\prime}=\otimes_{v} \chi_{v} \circ \operatorname{det}_{v}^{\prime}$ be an one-dimensional cuspidal automorphic representation of $\mathrm{GL}_{1}^{\prime}(\mathbb{A})$. Here $\chi_{v}$ are unitary characters of $k_{v}^{\times}$and $\chi$ is a unitary character of $\mathbb{A}^{\times} / k^{\times}$. By abuse of notation $\operatorname{det}_{v}^{\prime}$ denotes the determinant in $\mathrm{GL}_{2}\left(k_{v}\right)$ at split places and the reduced norm in $\mathrm{GL}_{1}^{\prime}\left(k_{v}\right) \cong D_{v}^{\times}$at nonsplit places, while $\operatorname{det}^{\prime}$ is the reduced norm of the simple algebra $\mathbb{A} \otimes_{k} D$. Then the global lift of $\chi \circ \operatorname{det}^{\prime}$ is just the one-dimensional representation $\chi \circ \operatorname{det}=$ $\otimes_{v} \chi_{v} \circ \operatorname{det}_{v}$ of $\mathrm{GL}_{2}(\mathbb{A})$. It belongs to the residual spectrum of $\mathrm{GL}_{2}$. At the non-split place the local lift of $\chi_{v} \circ \operatorname{det}_{v}^{\prime}$ is defined by the Jacquet-Langlands correspondence as in Theorem (8.1) of [10] to be the Steinberg representation of $\mathrm{GL}_{2}\left(k_{v}\right)$ twisted by $\chi_{v}$, i.e. the unique irreducible subrepresentation 
of the induced representation $\operatorname{Ind}_{\mathrm{GL}_{1}\left(k_{v}\right) \times \mathrm{GL}_{1}\left(k_{v}\right)}^{\mathrm{GL}_{2}\left(k_{v}\right)}\left(\chi_{v}|\cdot|{ }^{1 / 2} \otimes \chi_{v}|\cdot|^{-1 / 2}\right)$. We denote this representation by $S t_{\chi_{v}}$. Observe that by our definition in this case the global and local lift are not consistent. The reason is that the global lift is supposed to be in the discrete spectrum, while the local lift should preserve the Plancherel measure.

This Section is divided into three Subsections. First, in Subsection 1.1 we deal with the case of generic representation $\pi_{v} \cong \pi_{v}^{\prime}$ of $M_{0}\left(k_{v}\right) \cong M_{0}^{\prime}\left(k_{v}\right)$ at a split place $v$. If the global lift of $\pi^{\prime}$ is a cuspidal automorphic representation then this local component is indeed generic. For tempered generic representations the proof of the holomorphy and non-vanishing of normalized intertwining operators in the closure of the positive Weyl chamber is done in general for parabolic subgroups of any split classical group, but for non-tempered generic local components we specialize to parabolic subgroup $P_{0}\left(k_{v}\right)$ of $\mathrm{SO}_{8}\left(k_{v}\right)$ with the Levi factor $M_{0}\left(k_{v}\right)$. In Subsection 1.2 we settle the remaining split cases just for the Levi subgroup $M_{0}\left(k_{v}\right)$ of $\mathrm{SO}_{8}\left(k_{v}\right)$, i.e. local components at split places of those $\pi^{\prime}$ that lift to a residual spectrum representation of $M_{0}(\mathbb{A})$. This is done case by case. Finally, in Subsection 1.3 non-split places are considered. We show that the normalization in this case can be defined using the split case normalization factor of the local lift of $\pi_{v}^{\prime}$.

1.1. Generic split case. In what follows in the generic split case we use Shahidi's notation (see Section 1 of [45]) and work in the generality of split classical group $G$ defined over local field $k_{v}$ of characteristic zero. Let $T$ be a maximal split torus of $G$ and $\Phi$ the set of roots. Fix the Borel subgroup $B$ defining the set of positive roots $\Phi^{+}$and the set of simple roots $\Delta$. Let $W$ denote the Weyl group of $(G, T)$. For every proper subset $\theta$ of $\Delta$ let $P_{\theta}$ be the corresponding standard parabolic subgroup with the Levi decomposition $M_{\theta} N_{\theta}$, where $M_{\theta}$ is the Levi factor and $N_{\theta}$ the unipotent radical. Let $K_{v}$ be a fixed maximal compact subgroup of $G\left(k_{v}\right)$.

Let $X^{*}\left(M_{\theta}\right)$ be the $\mathbb{Z}$-module of $k_{v}$-rational characters of $M_{\theta}$ and

$$
\mathfrak{a}_{\theta}=\operatorname{Hom}_{\mathbb{Z}}\left(X^{*}\left(M_{\theta}\right), \mathbb{R}\right) \quad \text { and } \quad \mathfrak{a}_{\theta}^{*}=X^{*}\left(M_{\theta}\right) \otimes_{\mathbb{Z}} \mathbb{R} .
$$

The complexification of $\mathfrak{a}_{\theta}$ and $\mathfrak{a}_{\theta}^{*}$ denote by $\mathfrak{a}_{\theta, \mathbb{C}}$ and $\mathfrak{a}_{\theta, \mathbb{C}}^{*}$. Using the natural duality $\langle\cdot, \cdot\rangle$ of $\mathfrak{a}_{\theta, \mathbb{C}}^{*}$ and $\mathfrak{a}_{\theta, \mathbb{C}}$ the homomorphism $H_{P_{\theta}}: M_{\theta} \rightarrow \mathfrak{a}_{\theta}$ is defined by

$$
\exp \left\langle\chi, H_{P_{\theta}}(m)\right\rangle=|\chi(m)|_{v}
$$

for all rational characters $\chi \in X^{*}\left(M_{\theta}\right)$ where exp in the non-archimedean case denotes exponential function with the basis $q_{v}$ the number of elements of residual field of $k_{v}$.

For an irreducible admissible representation $\pi_{v}$ of $M_{\theta}\left(k_{v}\right)$ and $\underline{s} \in \mathfrak{a}_{\theta, \mathbb{C}}^{*}$ we form the induced representation

$$
I\left(\underline{s}, \pi_{v}\right)=\operatorname{Ind}_{M_{\theta}\left(k_{v}\right)}^{G\left(k_{v}\right)}\left(\pi_{v} \otimes \exp \left\langle\underline{s}, H_{P_{\theta}}(\cdot)\right\rangle\right),
$$


where, as remarked in the Introduction, $\operatorname{Ind}_{M_{\theta}\left(k_{v}\right)}^{G\left(k_{v}\right)}$ denotes induction from the standard parabolic subgroup $P_{\theta}\left(k_{v}\right)$. The representation of $M_{\theta}\left(k_{v}\right)$ is extended to the representation of $P_{\theta}\left(k_{v}\right)$ trivially on $N_{\theta}\left(k_{v}\right)$. Induction is normalized in such a way that the unitary representations of $M_{\theta}\left(k_{v}\right)$ give the unitary ones of $G\left(k_{v}\right)$.

The intertwining operators are defined for every $w \in W$ such that $w(\theta) \subset$ $\Delta$ by the integral

$$
A\left(\underline{s}, \pi_{v}, w\right) f_{\underline{s}, v}(g)=\int_{U\left(k_{v}\right) \cap w \bar{N}_{\theta}\left(k_{v}\right) w^{-1}} f_{\underline{s}, v}\left(w^{-1} n g\right) d n,
$$

where $f_{\underline{s}, v}$ is in the space of induced representation $I\left(\underline{s}, \pi_{v}\right), \bar{N}_{\theta}$ the unipotent radical of the opposite parabolic subgroup of $P_{\theta}$ and $U$ the unipotent radical of the Borel subgroup. The fixed Haar measure $d n$ on $N_{\theta}\left(k_{v}\right)$ is chosen as in Section 2 of [41]. Dependency of $f_{\underline{s}, v}$ on $\underline{s}$ is obtained using the compact picture with respect to $K_{v}$ as in Section II.1 of [38]. It is well known that this integral converges absolutely for real part of $\underline{s}$ far enough in the positive Weyl chamber and analytically continues to the meromorphic function of $\underline{s}$. If $P_{w \theta}$ is the standard parabolic subgroup corresponding to $w(\theta) \subset \Delta$ then the intertwining operator $A\left(\underline{s}, \pi_{v}, w\right)$ intertwines the representations $I\left(\underline{s}, \pi_{v}\right)$ and $I\left(w(\underline{s}), w\left(\pi_{v}\right)\right)$ whenever $\underline{s}$ is not a pole. Here $w(\underline{s})$ and $w\left(\pi_{v}\right)$ are the usual actions of the Weyl group obtained from the conjugation on the Levi factor.

Let $r_{\theta}$ be the adjoint representation of the Langlands dual L-group of $M_{\theta}$ on the Lie algebra of the L-group of $N_{\theta}$. It is completely reducible and let

$$
r_{\theta}=r_{1} \oplus \ldots \oplus r_{\ell},
$$

be its decomposition into irreducibles.

For the normalization of the intertwining operator it is crucial to consider the maximal proper parabolic subgroup case due to the Proposition of Langlands in the archimedean and Shahidi in the non-archimedean case giving the decomposition of $A\left(\underline{s}, \pi_{v}, w\right)$ into the product of such intertwining operators (Sections I.1.8 and IV.4.1 of [38]). The precise statement will be given later in Proposition 1.4 of this Section.

Suppose that $P=P_{\Delta \backslash\{\alpha\}}=M N$ is the Levi decomposition of the maximal proper standard parabolic subgroup of $G$ where $\alpha$ is a simple root. Then, unless $G=\mathrm{GL}_{n}$, the corresponding space $\mathfrak{a}_{\mathbb{C}}^{*}$ is one-dimensional. In any case we let

$$
\widetilde{\alpha}=\left\langle\rho_{P}, \alpha^{\vee}\right\rangle^{-1} \rho_{P},
$$

where $\rho_{P}$ equals half of the sum of positive roots of $G$ not being roots of $M$ and we write $s \widetilde{\alpha}=\widetilde{\alpha} \otimes s$ for $s \in \mathbb{C}$. In fact $\widetilde{\alpha}$ is the fundamental weight corresponding to $P$. For maximal proper standard parabolic subgroup of $\mathrm{GL}_{n}$ with the Levi factor isomorphic to $\mathrm{GL}_{n_{1}} \times \mathrm{GL}_{n_{2}}$ the space $\mathfrak{a}_{\mathbb{C}}^{*}$ is twodimensional. Nevertheless, tensoring by the appropriate power of the absolute 
value of the determinant reduces the consideration of intertwining operators to the one-dimensional subspace $s \widetilde{\alpha}=\left\langle\underline{s}, \alpha^{\vee}\right\rangle \widetilde{\alpha}$. Observe that in the maximal parabolic subgroup case there is at most one nontrivial element $w \in W$ such that $w(\Delta \backslash\{\alpha\}) \subset \Delta$. Then $w(s \widetilde{\alpha})=-s \widetilde{\alpha}$.

Let $\psi_{v}$ be a nontrivial continuous additive character of $k_{v}$. We define the normalizing factor for the intertwining operator attached to a $\psi_{v}$-generic representation $\pi_{v}$ of the Levi factor of a maximal parabolic subgroup in terms of $\mathrm{L}$-functions and $\varepsilon$-factors as

$$
r\left(s \widetilde{\alpha}, \pi_{v}, w\right)=\prod_{i=1}^{\ell} L\left(i s, \pi_{v}, r_{i}\right) L\left(1+i s, \pi_{v}, r_{i}\right)^{-1} \varepsilon\left(i s, \pi_{v}, r_{i}, \psi_{v}\right)^{-1}
$$

for $s \in \mathbb{C}$. Here, the L-functions and $\varepsilon$-factors are the ones defined by Shahidi in Section 7 of [45] using the Satake parametrization as in [46] to define the L-functions for unramified representations. This choice of the Satake parametrization is the reason of not having contragredients in (1.2). The normalized intertwining operator $N\left(s \widetilde{\alpha}, \pi_{v}, w\right)$ is given by

$$
A\left(s \widetilde{\alpha}, \pi_{v}, w\right)=r\left(s \widetilde{\alpha}, \pi_{v}, w\right) N\left(s \widetilde{\alpha}, \pi_{v}, w\right) .
$$

For an irreducible $\psi_{v}$-generic tempered representation $\pi_{v}$, the following Proposition gives the holomorphy and non-vanishing of these normalized operators in an open set slightly bigger than the closure of the positive Weyl chamber. The proof can be found in Lemma 1 and Lemma 2 of [54] having in mind that Conjecture A has been proved in Section 3 of [1] for the archimedean and in Section 4 of [7] for the non-archimedean case, while Assumption A follows from the standard module conjecture proved in [50] and [28] for the archimedean and in Section 2 of [7] and Section 1 of [40] for the non-archimedean case.

Proposition 1.3. Let $P=M N$ be a maximal proper standard parabolic subgroup of $G$ corresponding to $\Delta \backslash\{\alpha\}$ and $w$ the nontrivial element of the Weyl group $W$ such that $w(\Delta \backslash\{\alpha\}) \subset \Delta$. Let $\pi_{v}$ be an irreducible $\psi_{v}$-generic tempered representation of $M\left(k_{v}\right)$. Then the normalized intertwining operator $N\left(s \widetilde{\alpha}, \pi_{v}, w\right)$ is holomorphic and non-vanishing for $\operatorname{Re}(s)>-1 / \ell$ where $\ell$ is the length of the representation $r_{\Delta \backslash\{\alpha\}}$.

We consider now the case of general standard proper parabolic subgroup $P_{\theta}=M_{\theta} N_{\theta} \subset G$ for an irreducible $\psi_{v}$-generic representation $\pi_{v}$ of $M_{\theta}\left(k_{v}\right)$. First, we recall a decomposition of the intertwining operator $A\left(\underline{s}, \pi_{v}, w\right)$ (see Section 2.1 of [43]).

Proposition 1.4. For a subset $\theta \subset \Delta$ and $w \in W$ such that $w(\theta) \subset \Delta$ there exist simple roots $\alpha_{1}, \alpha_{2}, \ldots, \alpha_{n}$ having the following property: if the sequence $\theta_{1}, \ldots, \theta_{n}$ of subsets of $\Delta$ and the sequence $w_{1}, \ldots, w_{n}$ of elements of $W$ are defined inductively by $\theta_{1}=\theta, w_{i}$ is the unique nontrivial element of 
the Weyl group of $M_{\theta_{i} \cup\left\{\alpha_{i}\right\}}$ such that $w_{i}\left(\theta_{i}\right) \subset \theta_{i} \cup\left\{\alpha_{i}\right\}$, and $\theta_{i+1}=w_{i}\left(\theta_{i}\right)$. Then we have the following:

1. $w=w_{n} w_{n-1} \ldots w_{1}$ and $n$ is the length of $w$,

2. $A\left(\underline{s}, \pi_{v}, w\right)=A\left(\underline{s}_{n}, \pi_{v, n}, w_{n}\right) \ldots A\left(\underline{s}_{2}, \pi_{v, 2}, w_{2}\right) A\left(\underline{s}, \pi_{v}, w_{1}\right)$ where $\underline{s}_{i}=w_{i-1}\left(\underline{s}_{i-1}\right), \pi_{v, i}=w_{i-1}\left(\pi_{v, i-1}\right)$ for $i \geqslant 2$ and $\underline{s}_{1}=\underline{s}$, $\pi_{v, 1}=\pi_{v}$.

Observe that every $A\left(\underline{s}_{i}, \pi_{v, i}, w_{i}\right)$ is the intertwining operator in the maximal parabolic subgroup case for the Levi factor $M_{\theta_{i}} \subset M_{\theta_{i} \cup\left\{\alpha_{i}\right\}}$. The corresponding $s \in \mathbb{C}$ equals $\left\langle\underline{s}_{i}, \alpha_{i}^{\vee}\right\rangle$. Furthermore, if we define the subset $\Phi_{\theta, w}^{+}$of the set of positive roots modulo $Z_{\theta}$, the connected component of the center of $M_{\theta}$, by

$$
\Phi_{\theta, w}^{+}=\left\{\alpha \in \Phi^{+}: w \alpha<0\right\},
$$

then it consists of $n$ elements

$$
\Phi_{\theta, w}^{+}=\left\{\beta_{1}=\alpha_{1}, \beta_{2}=w_{1}^{-1} \alpha_{2}, \ldots, \beta_{n}=\left(w_{n-1} \ldots w_{1}\right)^{-1} \alpha_{n}\right\}
$$

and

$$
\left\langle\underline{s}_{i}, \alpha_{i}^{\vee}\right\rangle=\left\langle\underline{s}, \beta_{i}^{\vee}\right\rangle .
$$

Hence, the normalization of the intertwining operator $A\left(\underline{s}, \pi_{v}, w\right)$ is given according to the decomposition of Proposition 1.4 as

$$
r\left(\underline{s}, \pi_{v}, w\right)=\prod_{i=1}^{n} r\left(\left\langle\underline{s}_{i}, \alpha_{i}^{\vee}\right\rangle \widetilde{\alpha}_{i}, \pi_{v, i}, w_{i}\right)
$$

and the normalized operator by

$$
N\left(\underline{s}, \pi_{v}, w\right)=r\left(\underline{s}, \pi_{v}, w\right)^{-1} A\left(\underline{s}, \pi_{v}, w\right) .
$$

The decomposition of the normalized intertwining operator

$$
N\left(\underline{s}, \pi_{v}, w\right)=N\left(w_{1}(\underline{s}), w_{1}\left(\pi_{v}\right), w_{2}\right) N\left(\underline{s}, \pi_{v}, w_{1}\right)
$$

according to a not necessarily reduced decomposition of the Weyl group element $w=w_{2} w_{1}$ holds by Section 3 of [1] in the archimedean and Theorem 7.9 of [45] in the non-archimedean case. Next we prove the analogue of Proposition 1.3 for a $\psi_{v}$-generic tempered representation of $M_{\theta}\left(k_{v}\right)$.

Proposition 1.6. Let $P_{\theta}=M_{\theta} N_{\theta}$ be the proper standard parabolic subgroup of $G$ corresponding to $\theta$ and $w$ an element of the Weyl group $W$ such that $w(\theta) \subset \Delta$. Let $\pi_{v}$ be an irreducible $\psi_{v}$-generic tempered representation of $M_{\theta}\left(k_{v}\right)$. Then the normalized intertwining operator $N\left(\underline{s}, \pi_{v}, w\right)$ is holomorphic and non-vanishing for $\underline{s} \in \mathfrak{a}_{\theta, \mathbb{C}}^{*}$ such that $\left\langle\operatorname{Re}(\underline{s}), \alpha^{\vee}\right\rangle>-1 / \ell_{\alpha}$ for all $\alpha \in \Phi_{w, \theta}^{+}$, where $\ell_{\alpha}$ is the length of the corresponding adjoint representation $r_{\alpha}$ in the decomposition of Proposition 1.4. 
Proof. The normalized intertwining operator $N\left(\underline{s}, \pi_{v}, w\right)$ decomposes according to the decomposition $w=w_{n} \ldots w_{1}$ of Proposition 1.4 as

$$
N\left(\underline{s}, \pi_{v}, w\right)=N\left(\underline{s}_{n}, \pi_{v, n}, w_{n}\right) \ldots N\left(\underline{s}_{2}, \pi_{v, 2}, w_{2}\right) N\left(\underline{s}, \pi_{v}, w_{1}\right) .
$$

In order to prove holomorphy it is enough to prove the holomorphy of each factor. Factors are the maximal parabolic subgroup case intertwining operators for $\left\langle\underline{s}_{i}, \alpha_{i}^{\vee}\right\rangle \widetilde{\alpha}_{i}=\left\langle\underline{s}, \beta_{i}^{\vee}\right\rangle \widetilde{\alpha}_{i}$, where $\widetilde{\alpha}_{i}$ is the corresponding element of $\mathfrak{a}_{\theta, \mathbb{C}}^{*}$. Since $\Phi_{\theta, w}^{+}=\left\{\beta_{1}, \ldots, \beta_{n}\right\}$, the holomorphy at $\left\langle\operatorname{Re}(\underline{s}), \alpha^{\vee}\right\rangle>-1 / \ell_{\alpha}$ for all $\alpha \in \Phi_{w, \theta}^{+}$follows from Proposition 1.3. Non-vanishing is a consequence of holomorphy by Lemma 1.7 of [25].

Finally, we have to consider the non-tempered unitary $\psi_{v}$-generic representations. This will be done just for the representations $\pi_{v} \cong \pi_{1, v} \otimes \pi_{2, v}$ of $M_{0}\left(k_{v}\right) \cong \mathrm{GL}_{2}\left(k_{v}\right) \times \mathrm{GL}_{2}\left(k_{v}\right)$ in the split $\mathrm{SO}_{8}\left(k_{v}\right)$ which are the local components at $v$ of the global cuspidal automorphic representation $\pi \cong \pi_{1} \otimes \pi_{2}$ of $M_{0}(\mathbb{A})$.

Proposition 1.7. Let $P_{0}=M_{0} N_{0}$ be the standard parabolic subgroup of split group $\mathrm{SO}_{8}$ with the Levi factor $M_{0}$ isomorphic to $\mathrm{GL}_{2} \times \mathrm{GL}_{2}$. Let $\pi_{v} \cong \pi_{1, v} \otimes \pi_{2, v}$ be be the local component of a cuspidal automorphic representation $\pi \cong \pi_{1} \otimes \pi_{2}$ of $M_{0}(\mathbb{A})$. Then, for every $w \in W\left(M_{0}\right)$, the normalized intertwining operator $N\left(\underline{s}, \pi_{v}, w\right)$ is holomorphic and non-vanishing for $\underline{s}=\left(s_{1}, s_{2}\right)$ such that

- $\operatorname{Re}\left(s_{1}\right) \geqslant \operatorname{Re}\left(s_{2}\right) \geqslant 0$, i.e. the closure of the positive Weyl chamber,

- $0<s_{1}<1 / 2$ and $s_{2}=1 / 2$,

- $1 / 2<s_{1}<1$ and $s_{1}-s_{2}=1$.

PROOF. If a unitary generic representation $\pi_{i, v}$ of $\mathrm{GL}_{2}\left(k_{v}\right)$ is not tempered, then it is a complementary series, i.e. the fully induced representation of the form

$$
\pi_{i, v} \cong \operatorname{Ind}_{\mathrm{GL}_{1}\left(k_{v}\right) \times \mathrm{GL}_{1}\left(k_{v}\right)}^{\mathrm{GL}_{2}\left(k_{v}\right)}\left(\mu_{i, v}|\cdot|^{r_{i}} \otimes \mu_{i, v}|\cdot|^{-r_{i}}\right),
$$

where $\mu_{i, v}$ is a unitary character of $\mathrm{GL}_{1}\left(k_{v}\right)$ and $0<r_{i}<1 / 2$. However, since $\pi_{i, v}$ is the local component of a cuspidal automorphic representation of $\mathrm{GL}_{2}(\mathbb{A})$, the automorphy of the Gelbart-Jacquet lift of [9] implies that $r_{i}<1 / 4$.

Since intertwining operators are compatible with induction in stages the problem of holomorphy and non-vanishing is reduced to the tempered case. More precisely, there is a tempered representation $\tau_{v}$ of one of the Levi factors $\mathrm{GL}_{1}\left(k_{v}\right) \times \mathrm{GL}_{1}\left(k_{v}\right) \times \mathrm{GL}_{2}\left(k_{v}\right), \mathrm{GL}_{2}\left(k_{v}\right) \times \mathrm{GL}_{1}\left(k_{v}\right) \times \mathrm{GL}_{1}\left(k_{v}\right)$, $\mathrm{GL}_{1}\left(k_{v}\right) \times \mathrm{GL}_{1}\left(k_{v}\right) \times \mathrm{GL}_{1}\left(k_{v}\right) \times \mathrm{GL}_{1}\left(k_{v}\right)$, and an element $\underline{s}^{\prime}$ of the corresponding space $\mathfrak{a}_{\theta, \mathbb{C}}^{*}$ such that $I\left(\underline{s}, \pi_{v}\right) \cong I\left(\underline{s}^{\prime}, \tau_{v}\right)$ and hence, the holomorphy 
and non-vanishing of $N\left(\underline{s}, \pi_{v}, w\right)$ is equivalent to the holomorphy and nonvanishing of $N\left(\underline{s}^{\prime}, \tau_{v}, w\right)$. If $\underline{s}=\left(s_{1}, s_{2}\right)$, then in the three cases

$$
\underline{s}^{\prime}=\left\{\begin{array}{l}
\left(s_{1}+r_{1}, s_{1}-r_{1}, s_{2}\right), \\
\left(s_{1}, s_{2}+r_{2}, s_{2}-r_{2}\right), \\
\left(s_{1}+r_{1}, s_{1}-r_{1}, s_{2}+r_{2}, s_{2}-r_{2}\right) .
\end{array}\right.
$$

Since all the representations $r_{\alpha}$ in the three cases are irreducible, by Proposition 1.6, it is enough to check that if $\underline{s}=\left(s_{1}, s_{2}\right)$ is as in the statement of the Proposition, then $\left\langle\operatorname{Re}\left(\underline{s}^{\prime}\right), \beta^{\vee}\right\rangle>-1$ for every $\beta \in \Phi_{\theta, w}^{+}$. That is a straightforward check using the bound $0<r_{i}<1 / 4$.

REMARK 1.8. The normalizing factors are defined here using the Langlands-Shahidi method as in [45] for the non-archimedean and in [44] for the archimedean case. However, besides the holomorphy and non-vanishing properties of the local normalized intertwining operators which are important in our application, these factors satisfy Theorem 2.1 of [1] by Section 7 of [45] for the non-archimedean and Section 3 of [1] for the archimedean case.

1.2. Remaining split cases. The previous Subsection solves the problem of the holomorphy and non-vanishing of the local normalized intertwining operators in the closure of the positive Weyl chamber for the generic unitary representations of the Levi factor $M_{0}\left(k_{v}\right) \cong \mathrm{GL}_{2}\left(k_{v}\right) \times \mathrm{GL}_{2}\left(k_{v}\right)$ of the group $G_{2}^{\prime}\left(k_{v}\right) \cong \mathrm{SO}_{8}\left(k_{v}\right)$ at split places $v$. In this Subsection we prove the same result in the non-generic split cases needed in the sequel. These cases occur at split places when the global lift of a cuspidal automorphic representation of the Levi factor $M_{0}^{\prime}(\mathbb{A})$ is not cuspidal. This happens in the case of $M_{0}^{\prime} \cong \mathrm{GL}_{1}^{\prime} \times \mathrm{GL}_{1}^{\prime}$ if at least one of the cuspidal automorphic representations of $\mathrm{GL}_{1}^{\prime}(\mathbb{A})$ is one-dimensional. However, first we give the general idea how to define the normalizing factors for certain non-generic representations and prove the holomorphy and non-vanishing of the normalized intertwining operators. This will be done as in Lemma I.8 of [37]. In the sequel we refer to this general idea as the general overview of the proof.

For the moment let $G$ be any classical split group defined over $k_{v}$ as in the previous Subsection. We use all the notation introduced there. Let $P=M N$ be the standard proper parabolic subgroup of $G$ defined over $k_{v}$ corresponding to $\theta \subset \Delta$ and let $\pi_{v}$ be an irreducible unitary non-generic representation of $M\left(k_{v}\right)$. Assume that there exists a standard parabolic subgroup of $M$ with the Levi factor $L$ corresponding to the subset $\theta^{\prime} \subset \theta \subset \Delta$, an irreducible tempered generic representation $\tau_{v}$ of $L\left(k_{v}\right)$ and $\underline{s}^{\prime} \in \mathfrak{a}_{\theta^{\prime}, \mathbb{C}}^{*}$ such that $\pi_{v}$ is isomorphic to the unique irreducible subrepresentation of

$$
I_{L}^{M}\left(\underline{s}^{\prime}, \tau_{v}\right)=\operatorname{Ind}_{L\left(k_{v}\right)}^{M\left(k_{v}\right)}\left(\tau_{v} \otimes \exp \left\langle\underline{s}^{\prime}, H_{L}^{M}(\cdot)\right\rangle\right),
$$

where $H_{L}^{M}: L \rightarrow \mathfrak{a}_{L}$ is the homomorphism defined in the generic split case. Then, for every element $w$ of the Weyl group such that $w(\theta) \subset \Delta$, the following 
diagram is commutative:

$$
\begin{gathered}
I\left(\underline{s}, \pi_{v}\right) \hookrightarrow I\left(\underline{s}+\underline{s}^{\prime}, \tau_{v}\right) \\
A\left(\underline{s}, \pi_{v}, w\right) \downarrow \\
I\left(w(\underline{s}), w\left(\pi_{v}\right)\right) \hookrightarrow I\left(w\left(\underline{s}+\underline{s}^{\prime}\right), w\left(\tau_{v}\right)\right),
\end{gathered}
$$

where $\underline{s}$ is embedded into $\mathfrak{a}_{\theta^{\prime}}^{*}, \mathbb{C}$. In other words, $A\left(\underline{s}, \pi_{v}, w\right)$ is the restriction of $A\left(\underline{s}+\underline{s}^{\prime}, \tau_{v}\right)$ to $I\left(\underline{s}, \pi_{v}\right)$. Hence, the normalizing factor for $A\left(\underline{s}, \pi_{v}, w\right)$ is defined to be

$$
r\left(\underline{s}, \pi_{v}, w\right)=r\left(\underline{s}+\underline{s}^{\prime}, \tau_{v}, w\right)
$$

and the normalized operator $N\left(\underline{s}, \pi_{v}, w\right)$ is actually the restriction of $N(\underline{s}+$ $\left.\underline{s}^{\prime}, \tau_{v}, w\right)$ to $I\left(\underline{s}, \pi_{v}\right)$.

In order to prove the holomorphy and non-vanishing of $N\left(\underline{s}, \pi_{v}, w\right)$ we follow the proof of Lemma I.8 of [37]. Since $\pi_{v}$ is the unique irreducible subrepresentation of $I_{L}^{M}\left(\underline{s}^{\prime}, \tau_{v}\right)$, there is an element $w^{\prime}$ of the Weyl group such that $\pi_{v}$ is the image of the $M\left(k_{v}\right)$ normalized intertwining operator $N\left(w^{\prime-1}\left(\underline{s}^{\prime}\right), w^{\prime-1}\left(\tau_{v}\right), w^{\prime}\right)$. Observe that $w^{\prime}(\underline{s})=\underline{s}$. Then, $N\left(\underline{s}, \pi_{v}, w\right)$ fits into the following commutative diagram:

$$
\begin{aligned}
& I\left(\underline{s}, \pi_{v}\right) \stackrel{N\left(\underline{s}+w^{\prime-1}\left(\underline{s}^{\prime}\right), w^{\prime-1}\left(\tau_{v}\right), w^{\prime}\right)}{\longleftarrow} I\left(\underline{s}+w^{\prime-1}\left(\underline{s}^{\prime}\right), w^{\prime-1}\left(\tau_{v}\right)\right) \\
& N\left(\underline{s}, \pi_{v}, w\right) \downarrow \quad \downarrow N\left(\underline{s}+w^{\prime-1}\left(\underline{s}^{\prime}\right), w^{\prime-1}\left(\tau_{v}\right), w w^{\prime}\right) \\
& I\left(w(\underline{s}), w\left(\pi_{v}\right)\right) \quad \hookrightarrow \quad I\left(w\left(\underline{s}+\underline{s}^{\prime}\right), w\left(\tau_{v}\right)\right) .
\end{aligned}
$$

Now, if $\underline{s}+w^{\prime-1}\left(\underline{s}^{\prime}\right) \in \mathfrak{a}_{\theta^{\prime}, \mathbb{C}}^{*}$ satisfies the inequalities of Proposition 1.6 for $w w^{\prime}$, then the right vertical arrow is holomorphic and non-vanishing. Since the upper horizontal arrow is surjective, the commutativity of the diagram implies that $N\left(\underline{s}, \pi_{v}, w\right)$ is holomorphic and non-vanishing for such $\underline{s}$.

If $\underline{s}+w^{\prime-1}\left(\underline{s}^{\prime}\right) \in \mathfrak{a}_{\theta^{\prime}, \mathbb{C}}^{*}$ does not satisfy the inequalities of Proposition 1.6 for $w w^{\prime}$, then there is an element $w^{\prime \prime}$ of the Weyl group depending on $\underline{s}$ such that $w^{\prime \prime-1}\left(\underline{s}+w^{\prime-1}\left(\underline{s}^{\prime}\right)\right)$ satisfies those inequalities for $w w^{\prime} w^{\prime \prime}$. Then, $N\left(\underline{s}, \pi_{v}, w\right)$ fits into the following commutative diagram:

$$
\begin{aligned}
& I\left(s, \pi_{v}\right) \longleftarrow \quad N \quad I\left(w^{\prime \prime-1}\left(\underline{s}+w^{\prime-1}\left(\underline{s}^{\prime}\right)\right), w^{\prime \prime-1} w^{\prime-1}\left(\tau_{v}\right)\right) \\
& N\left(\underline{s}, \pi_{v}, w\right) \downarrow \quad N\left(w^{\prime \prime-1}\left(\underline{s}+w^{\prime-1}\left(\underline{s}^{\prime}\right)\right), w^{\prime \prime-1} w^{\prime-1}\left(\tau_{v}\right), w w^{\prime} w^{\prime \prime}\right) \downarrow \\
& I\left(w(\underline{s}), w\left(\pi_{v}\right)\right) \quad \hookrightarrow \quad I\left(w\left(\underline{s}+\underline{s}^{\prime}\right), w\left(\tau_{v}\right)\right),
\end{aligned}
$$

where $N=N\left(w^{\prime \prime-1}\left(\underline{s}+w^{\prime-1}\left(\underline{s}^{\prime}\right)\right), w^{\prime \prime-1} w^{\prime-1}\left(\tau_{v}\right), w^{\prime} w^{\prime \prime}\right)$. 
The right vertical arrow is holomorphic and non-vanishing by the choice of $w^{\prime \prime}$. Hence, the commutativity of the diagram would imply the holomorphy and non-vanishing of $N\left(\underline{s}, \pi_{v}, w\right)$ for such $\underline{s}$ if we were able to prove that the upper horizontal arrow is surjective. That is what we do in the required cases.

In this paper the non-generic representation $\pi_{v} \cong \pi_{v}^{\prime}$ considered in the general overview above will be the local component at a split place $v$ of a cuspidal automorphic representation $\pi^{\prime} \cong \pi_{1}^{\prime} \otimes \pi_{2}^{\prime}$ of the Levi factor $M_{0}^{\prime}(\mathbb{A}) \cong$ $\mathrm{GL}_{1}^{\prime}(\mathbb{A}) \times \mathrm{GL}_{1}^{\prime}(\mathbb{A})$ of $G_{2}^{\prime}(\mathbb{A})$. If the global lift $\pi$ of $\pi^{\prime}$ is a cuspidal automorphic representation of $M_{0}(\mathbb{A})$ then all the local components are generic. Therefore, we assume that $\pi$ is not cuspidal, i.e. at least one of the representations $\pi_{1}^{\prime}$ and $\pi_{2}^{\prime}$ is one-dimensional. In the rest of this Subsection we treat separately the case when both representations are one-dimensional and the case when one of them is one-dimensional. In accordance we the following Section 2, the two cases are called case $\mathrm{C}$ and case $\mathrm{B}$, respectively.

Let $\pi_{i}^{\prime} \cong \chi_{i}$ o $\operatorname{det}^{\prime}$ for $i=1,2$, where $\chi_{i}$ are unitary characters of $\mathbb{A}^{\times} / k^{\times}$. Then the local components at a split place $v$ are $\pi_{i, v}^{\prime} \cong \pi_{i, v} \cong \chi_{i, v} \circ \operatorname{det}_{v}$ where $\chi_{i, v}$ are unitary characters of $k_{v}^{\times}$. Hence, in this case $\pi_{v} \cong\left(\chi_{1, v} \circ \operatorname{det}_{v}\right) \otimes$ $\left(\chi_{2, v} \circ \operatorname{det}_{v}\right)$ is a non-generic representation of the Levi factor $M_{0}\left(k_{v}\right) \cong$ $\mathrm{GL}_{2}\left(k_{v}\right) \times \mathrm{GL}_{2}\left(k_{v}\right)$ of $\mathrm{SO}_{8}\left(k_{v}\right)$. Let $T \cong \mathrm{GL}_{1} \times \mathrm{GL}_{1} \times \mathrm{GL}_{1} \times \mathrm{GL}_{1}$ be the maximal split torus of $\mathrm{SO}_{8}$ defined over $k_{v}$. Then $\pi_{v}$ is the unique irreducible subrepresentation of

$$
\operatorname{Ind}_{T\left(k_{v}\right)}^{M_{0}\left(k_{v}\right)}\left(\left.\left.\chi_{1, v}|\cdot|^{-1 / 2} \otimes \chi_{1, v}|\cdot|\right|^{1 / 2} \otimes \chi_{2, v}|\cdot|^{-1 / 2} \otimes \chi_{2, v}|\cdot|\right|^{1 / 2}\right) .
$$

In the notation of the general overview above $L=T, M=M_{0}$,

$$
\tau_{v}=\chi_{1, v} \otimes \chi_{1, v} \otimes \chi_{2, v} \otimes \chi_{2, v} \quad \text { and } \quad \underline{s}^{\prime}=(-1 / 2,1 / 2,-1 / 2,1 / 2) .
$$

Therefore, for $\underline{s}=\left(s_{1}, s_{2}\right) \in \mathfrak{a}_{M_{0}^{\prime}, \mathbb{C}}^{*}$ and an element $w \in W\left(M_{0}^{\prime}\right)$, the normalizing factor is defined to be

$$
r\left(\underline{s}, \pi_{v}, w\right)=r\left(\left(s_{1}-1 / 2, s_{1}+1 / 2, s_{2}-1 / 2, s_{2}+1 / 2\right), \tau_{v}, w\right)
$$

and the normalized intertwining operator $N\left(\underline{s}, \pi_{v}, w\right)$ is the restriction of

$$
N\left(\left(s_{1}-1 / 2, s_{1}+1 / 2, s_{2}-1 / 2, s_{2}+1 / 2\right), \tau_{v}, w\right)
$$

to $I\left(\underline{s}, \pi_{v}\right)$. More precisely, for the maximal parabolic subgroup case $\mathrm{GL}_{2}\left(k_{v}\right) \times \mathrm{GL}_{2}\left(k_{v}\right) \subset \mathrm{GL}_{4}\left(k_{v}\right)$ the normalizing factor equals

$$
r\left(\left(s_{1}, s_{2}\right),\left(\chi_{1, v} \circ \operatorname{det}_{v}\right) \otimes\left(\chi_{2, v} \circ \operatorname{det}_{v}\right), w_{1}\right)=r\left(s_{1}-s_{2}, \chi_{1, v} \chi_{2, v}^{-1}\right),
$$

where $w_{1}$ is the unique nontrivial Weyl group element of this case and

$$
r\left(s, \chi_{v}\right)=\frac{L\left(s, \chi_{v}\right) L\left(s-1, \chi_{v}\right)}{L\left(s+2, \chi_{v}\right) L\left(s+1, \chi_{v}\right) \varepsilon\left(s+1, \chi_{v}, \psi_{v}\right) \varepsilon\left(s, \chi_{v}, \psi_{v}\right)^{2} \varepsilon\left(s-1, \chi_{v}, \psi_{v}\right)} .
$$


For the maximal parabolic subgroup case $\mathrm{GL}_{2}\left(k_{v}\right) \subset \mathrm{SO}_{4}\left(k_{v}\right)$ the normalizing factor equals

$$
\begin{aligned}
& r\left(\left(s_{1}, s_{2}\right),\left(\chi_{1, v} \circ \operatorname{det}_{v}\right) \otimes\left(\chi_{2, v} \circ \operatorname{det}_{v}\right), w_{2}\right) \\
& =r\left(2 s_{2} \widetilde{\alpha}, \chi_{2, v} \circ \operatorname{det}_{v}, w_{2}\right)=\frac{L\left(2 s_{2}, \chi_{2, v}^{2}\right)}{L\left(2 s_{2}+1, \chi_{2, v}^{2}\right) \varepsilon\left(2 s_{2}, \chi_{2, v}^{2}, \psi_{v}\right)},
\end{aligned}
$$

where $w_{2}$ is the unique nontrivial Weyl group element of this case.

Proposition 1.12. The normalized intertwining operator

$$
N\left(\left(s_{1}, s_{2}\right),\left(\chi_{1, v} \circ \operatorname{det}_{v}\right) \otimes\left(\chi_{2, v} \circ \operatorname{det}_{v}\right), w\right)
$$

defined above is holomorphic and non-vanishing for all $w \in W\left(M_{0}^{\prime}\right)$ at $\underline{s}=$ $\left(s_{1}, s_{2}\right)$ such that

- $\operatorname{Re}\left(s_{1}\right) \geqslant \operatorname{Re}\left(s_{2}\right) \geqslant 0$ except $\operatorname{Re}\left(s_{1}\right)=\operatorname{Re}\left(s_{2}\right)=0$, i.e. in the closure of the positive Weyl chamber except at the origin,

- $0<s_{1}<1 / 2$ and $s_{2}=1 / 2$,

- $1<s_{1}<2$ and $s_{1}-s_{2}=2$ except at $\left(s_{1}, s_{2}\right)=(3 / 2,-1 / 2)$.

At the exceptional point $\left(s_{1}, s_{2}\right)=(3 / 2,-1 / 2)$ it is always holomorphic and non-vanishing for $w \in\left\{1, w_{1}, w_{2} w_{1}, w_{1} w_{2} w_{1}\right\}$. Moreover, for $w \in$ $\left\{w_{2}, w_{1} w_{2}, w_{2} w_{1} w_{2}, w_{1} w_{2} w_{1} w_{2}\right\}$ it is holomorphic and non-vanishing at $\left(s_{1}, s_{2}\right)=(3 / 2,-1 / 2)$ if $\chi_{2, v}^{2}$ is nontrivial.

Proof. By the discussion preceding this Proposition, in the notation of the general overview

$$
\tau_{v}=\chi_{1, v} \otimes \chi_{1, v} \otimes \chi_{2, v} \otimes \chi_{2, v} \quad \text { and } \quad \underline{s}^{\prime}=(-1 / 2,1 / 2,-1 / 2,1 / 2) .
$$

Then $w^{\prime}$ is the element of the Weyl group corresponding to the permutation $w^{\prime}=(1,2)(3,4)$, where $\left(i_{1}, \ldots, i_{l}\right)$ denotes the cycle mapping $i_{1} \rightarrow i_{2} \rightarrow$ $\ldots \rightarrow i_{l} \rightarrow i_{1}$. The Weyl group element corresponding to the permutation $p$ acts on $\mathfrak{a}_{L, \mathbb{C}}^{*}$ as $\left(s_{1}, \ldots, s_{k}\right) \rightarrow\left(s_{p^{-1}(1)}, \ldots, s_{p^{-1}(k)}\right)$ and analogously on the representations. Hence,

$$
\underline{s}+w^{\prime-1}\left(\underline{s}^{\prime}\right)=\left(s_{1}+1 / 2, s_{1}-1 / 2, s_{2}+1 / 2, s_{2}-1 / 2\right) .
$$

If $\operatorname{Re}\left(s_{1}\right)>\operatorname{Re}\left(s_{2}\right) \geqslant 0$ the inequalities of Proposition 1.6 are satisfied for every element $w \in W\left(M_{0}^{\prime}\right)$ and the general overview above implies the holomorphy and non-vanishing.

For the closure of the positive Weyl chamber the case $s=\operatorname{Re}\left(s_{1}\right)=$ $\operatorname{Re}\left(s_{2}\right)>0$ remains. We can assume $s_{1}$ and $s_{2}$ are real because otherwise the unitary twist can be incorporated into the representations. The inequalities of Proposition 1.6 fail to be satisfied because the difference

$$
\left(s_{1}-1 / 2\right)-\left(s_{2}+1 / 2\right)=-1,
$$


and it should be strictly greater than -1 . Hence, take $w^{\prime \prime}=(1)(2,3)(4)$. Then

$$
w^{\prime \prime-1}\left(\underline{s}+w^{\prime-1}\left(\underline{s}^{\prime}\right)\right)=(s+1 / 2, s+1 / 2, s-1 / 2, s-1 / 2),
$$

which satisfies the inequalities of Proposition 1.6 for every $w \in W\left(M_{0}^{\prime}\right)$, and

$$
w^{\prime \prime-1} w^{\prime-1}\left(\tau_{v}\right) \cong \chi_{1, v} \otimes \chi_{2, v} \otimes \chi_{1, v} \otimes \chi_{2, v} .
$$

Therefore, by the general overview, it is enough to check that the image of

$$
N\left((s+1 / 2, s+1 / 2, s-1 / 2, s-1 / 2), \chi_{1, v} \otimes \chi_{2, v} \otimes \chi_{1, v} \otimes \chi_{2, v}, w^{\prime} w^{\prime \prime}\right)
$$

is exactly $I\left(\underline{s}, \pi_{v}\right)$. This is a consequence of the following decomposition

$$
\begin{gathered}
\operatorname{Ind}_{T\left(k_{v}\right)}^{\mathrm{SO}_{8}\left(k_{v}\right)}\left(\left.\left.\chi_{1, v}|\cdot|^{s+1 / 2} \otimes \chi_{2, v}|\cdot|\right|^{s+1 / 2} \otimes \chi_{1, v}|\cdot|^{s-1 / 2} \otimes \chi_{2, v}|\cdot|\right|^{s-1 / 2}\right) \\
\downarrow \\
\operatorname{Ind}_{T\left(k_{v}\right)}^{\mathrm{SO}_{8}\left(k_{v}\right)}\left(\chi_{2, v}|\cdot|^{s+1 / 2} \otimes \chi_{1, v}|\cdot|^{s+1 / 2} \otimes \chi_{1, v}|\cdot|^{s-1 / 2} \otimes \chi_{2, v}|\cdot|^{s-1 / 2}\right) \\
\downarrow \\
\operatorname{Ind}_{\mathrm{GL}_{1}\left(k_{v}\right) \times \mathrm{GL}_{2}\left(k_{v}\right) \times \mathrm{GL}_{1}\left(k_{v}\right)}^{\mathrm{SO}_{8}\left(k_{v}\right)}\left(\chi_{2, v}|\cdot|^{s+1 / 2} \otimes\left(\chi_{1, v} \circ \operatorname{det}_{v}\right)|\cdot|^{s} \otimes \chi_{2, v}|\cdot|^{s-1 / 2}\right) \\
\downarrow \\
\operatorname{Ind}_{\mathrm{GL}_{2}\left(k_{v}\right) \times \mathrm{GL}_{1}\left(k_{v}\right) \times \mathrm{GL}_{1}\left(k_{v}\right)}^{\mathrm{SO}_{8}\left(k_{v}\right)}\left(\left.\left(\chi_{1, v} \circ \operatorname{det}_{v}\right)|\cdot|^{s} \otimes \chi_{2, v}|\cdot|\right|^{s+1 / 2} \otimes \chi_{2, v}|\cdot|^{s-1 / 2}\right) \\
\downarrow \\
\operatorname{Ind}_{\mathrm{GL}_{2}\left(k_{v}\right) \times \mathrm{GL}_{2}\left(k_{v}\right)}^{\mathrm{SO}_{8}\left(k_{v}\right)}\left(\left(\chi_{1, v} \circ \operatorname{det}_{v}\right)|\cdot|^{s} \otimes\left(\chi_{2, v} \circ \operatorname{det}_{v}\right)|\cdot|^{s}\right),
\end{gathered}
$$

where the first and the third operators are isomorphisms and the second and the fourth are surjective.

The case $0<s_{1}<1 / 2$ and $s_{2}=1 / 2$ is settled in the same way since again only the difference

$$
\left(s_{1}-1 / 2\right)-\left(s_{2}+1 / 2\right)=s_{1}-3 / 2<-1
$$

fails to satisfy the inequalities of Proposition 1.6.

In the case $1<s_{1}<2$ and $s_{1}-s_{2}=2$ let $z$ be the new variable given by $s_{1}=z+1$. Then $s_{2}=z-1$ and $0<z<1$. In the new variable

$$
\underline{s}+w^{\prime-1}\left(\underline{s}^{\prime}\right)=(z+3 / 2, z+1 / 2, z-1 / 2, z-3 / 2) .
$$

For $w \in\left\{1, w_{1}, w_{2} w_{1}, w_{1} w_{2} w_{1}\right\}$ the inequalities of Proposition 1.6 are satisfied for all $0<z<1$. For the remaining $w$ they are satisfied if $z>1 / 2$. But if $0<z \leqslant 1 / 2$ the sum

$$
(z-1 / 2)+(z-3 / 2)=2 z-2 \leqslant-1 .
$$

Nevertheless, taking $w^{\prime \prime}=w_{2}$ we obtain

$$
w^{\prime \prime-1}\left(\underline{s}+w^{\prime-1}\left(\underline{s}^{\prime}\right)\right)=(z+3 / 2, z+1 / 2,-z+3 / 2,-z+1 / 2)
$$


which satisfies the inequalities of Proposition 1.6 for $0<z \leqslant 1 / 2$, and

$$
w^{\prime \prime-1} w^{\prime-1}\left(\tau_{v}\right) \cong \chi_{1, v} \otimes \chi_{1, v} \otimes \chi_{2, v}^{-1} \otimes \chi_{2, v}^{-1} .
$$

Therefore, by the general overview, it is enough to check that the image of

$$
N\left((z+3 / 2, z+1 / 2,-z+3 / 2,-z+1 / 2), \chi_{1, v} \otimes \chi_{1, v} \otimes \chi_{2, v}^{-1} \otimes \chi_{2, v}^{-1}, w^{\prime} w^{\prime \prime}\right)
$$

is exactly $I\left(\underline{s}, \pi_{v}\right)$. This is certainly true if either $z \neq 1 / 2$ or $z=1 / 2$ and $\chi_{2, v}^{2}$ is nontrivial because then the normalized intertwining operator corresponding to $w^{\prime \prime}$ acts on the irreducible induced representation and thus it is an isomorphism. Since $z=1 / 2$ corresponds to $\left(s_{1}, s_{2}\right)=(3 / 2,-1 / 2)$ the last case of the Proposition is settled.

Let $\pi_{1}^{\prime} \cong \chi_{1} \circ$ det $^{\prime}$ be one-dimensional and $\pi_{2}^{\prime}$ not one-dimensional cuspidal automorphic representation of $\mathrm{GL}_{1}^{\prime}(\mathbb{A})$, where $\chi_{1}$ is a unitary character of $\mathbb{A}^{\times} / k^{\times}$. The other case, $\pi_{1}^{\prime}$ not one-dimensional and $\pi_{2}^{\prime}$ one-dimensional, is settled in the completely same way. The local components of $\pi_{1}^{\prime}$ at split places are $\pi_{1, v}^{\prime} \cong \pi_{1, v} \cong \chi_{1, v} \circ \operatorname{det}_{v}$, where $\chi_{1, v}$ is a unitary character of $k_{v}^{\times}$, while the local components of $\pi_{2}^{\prime}$ at split places are the generic unitary representations $\pi_{2, v}^{\prime} \cong \pi_{2, v}$ of $\mathrm{GL}_{2}\left(k_{v}\right)$ because they are the local components of the global lift of $\pi_{2}^{\prime}$ which is cuspidal automorphic. Hence, if not tempered, $\pi_{2, v}$ is a complementary series representation

$$
\pi_{2, v} \cong \operatorname{Ind}_{\mathrm{GL}_{1}\left(k_{v}\right) \times \mathrm{GL}_{1}\left(k_{v}\right)}^{\mathrm{GL}_{2}\left(k_{v}\right)}\left(\mu_{v}|\cdot|^{r} \otimes \mu_{v}|\cdot|^{-r}\right),
$$

where $0<r<1 / 2$ and $\mu_{v}$ is a unitary character of $\mathrm{GL}_{1}\left(k_{v}\right)$. In this case $\pi_{v} \cong\left(\chi_{1, v} \circ \operatorname{det}_{v}\right) \otimes \pi_{2, v}$ is the unique irreducible subrepresentation of

$$
\operatorname{Ind}_{\mathrm{GL}_{1}\left(k_{v}\right) \times \mathrm{GL}_{1}\left(k_{v}\right) \times \mathrm{GL}_{2}\left(k_{v}\right)}^{M_{0}\left(k_{v}\right)}\left(\chi_{1, v}|\cdot|^{-1 / 2} \otimes \chi_{1, v}|\cdot|^{1 / 2} \otimes \pi_{2, v}\right),
$$

which is isomorphic to

$$
\operatorname{Ind}_{T\left(k_{v}\right)}^{M_{0}\left(k_{v}\right)}\left(\chi_{1, v}|\cdot|^{-1 / 2} \otimes \chi_{1, v}|\cdot|^{1 / 2} \otimes \mu_{v}|\cdot|^{r} \otimes \mu_{v}|\cdot|^{-r}\right)
$$

if $\pi_{2, v}$ is a complementary series. More precisely, in terms of the general overview above, if $\pi_{2, v}$ is tempered $L=\mathrm{GL}_{1} \times \mathrm{GL}_{1} \times \mathrm{GL}_{2}, M=M_{0}$,

$$
\tau_{v} \cong \chi_{1, v} \otimes \chi_{1, v} \otimes \pi_{2, v} \quad \text { and } \quad \underline{s}^{\prime}=(-1 / 2,1 / 2,0),
$$

while if $\pi_{2, v}$ is a complementary series $L=T, M=M_{0}$,

$$
\tau_{v} \cong \chi_{1, v} \otimes \chi_{1, v} \otimes \mu_{v} \otimes \mu_{v} \quad \text { and } \quad \underline{s}^{\prime}=(-1 / 2,1 / 2, r,-r) .
$$

Therefore, for $\underline{s}=\left(s_{1}, s_{2}\right) \in \mathfrak{a}_{M_{0}^{\prime}, \mathbb{C}}^{*}$ and $w \in W\left(M_{0}^{\prime}\right)$ the normalizing factor is defined to be

$$
r\left(\underline{s}, \pi_{v}, w\right)=r\left(\left(s_{1}-1 / 2, s_{1}+1 / 2, s_{2}\right), \chi_{1, v} \otimes \chi_{1, v} \otimes \pi_{2, v}, w\right)
$$

if $\pi_{2, v}$ is tempered and

$$
r\left(\underline{s}, \pi_{v}, w\right)=r\left(\left(s_{1}-1 / 2, s_{1}+1 / 2, s_{2}+r, s_{2}-r\right), \chi_{1, v} \otimes \chi_{1, v} \otimes \mu_{v} \otimes \mu_{v}, w\right)
$$


if $\pi_{2, v}$ is a complementary series. Since a complementary series is a fully induced representation, the latter normalizing factor is also equal to

$$
r\left(\left(s_{1}-1 / 2, s_{1}+1 / 2, s_{2}\right), \chi_{1, v} \otimes \chi_{1, v} \otimes \pi_{2, v}, w\right),
$$

thus giving the uniform formula at all split places which is more suitable for the calculations in the next Section. Nevertheless, here we prefer to write the normalizing factor using tempered representations only. For the maximal parabolic subgroup case $\mathrm{GL}_{2}\left(k_{v}\right) \times \mathrm{GL}_{2}\left(k_{v}\right) \subset \mathrm{GL}_{4}\left(k_{v}\right)$ the normalizing factor equals

$$
\begin{aligned}
& r\left(\left(s_{1}, s_{2}\right),\left(\chi_{1, v} \circ \operatorname{det}_{v}^{\prime}\right) \otimes \pi_{2, v}, w_{1}\right) \\
& =\frac{L\left(s-1 / 2, \chi_{1, v} \widetilde{\pi}_{2, v}\right)}{L\left(s+3 / 2, \chi_{1, v} \widetilde{\pi}_{2, v}\right) \varepsilon\left(s+1 / 2, \chi_{1, v} \widetilde{\pi}_{2, v}\right) \varepsilon\left(s-1 / 2, \chi_{1, v} \widetilde{\pi}_{2, v}\right)},
\end{aligned}
$$

where $s=s_{1}-s_{2}$ and $w_{1}$ is the unique nontrivial element of the Weyl group of this case. For the maximal parabolic subgroup case $\mathrm{GL}_{2}\left(k_{v}\right) \subset \mathrm{SO}_{4}\left(k_{v}\right)$ and representation $\chi_{v} \circ \operatorname{det}_{v}$ of $\mathrm{GL}_{2}\left(k_{v}\right)$ the normalizing factor corresponding to the unique nontrivial Weyl group element $w_{2}$ of this case is given again by (1.11).

The normalized intertwining operator $N\left(\underline{s}, \pi_{v}, w\right)$ is the restriction of

$$
N\left(\left(s_{1}-1 / 2, s_{1}+1 / 2, s_{2}\right), \chi_{1, v} \otimes \chi_{1, v} \otimes \pi_{2, v}, w\right)
$$

to $I\left(\underline{s}, \pi_{v}\right)$. Again, this normalized intertwining operator is equal to

$$
N\left(\left(s_{1}-1 / 2, s_{1}+1 / 2, s_{2}+r, s_{2}-r\right), \chi_{1, v} \otimes \chi_{1, v} \otimes \mu_{v} \otimes \mu_{v}, w\right)
$$

if $\pi_{2, v}$ is a complementary series.

Proposition 1.14. For every $w \in W\left(M_{0}^{\prime}\right)$, the normalized intertwining operator

$$
N\left(\left(s_{1}, s_{2}\right),\left(\chi_{1, v} \circ \operatorname{det}_{v}\right) \otimes \pi_{2, v}, w\right)
$$

defined above is holomorphic and non-vanishing for $\underline{s}=\left(s_{1}, s_{2}\right)$ such that

- $\operatorname{Re}\left(s_{1}\right) \geqslant \operatorname{Re}\left(s_{2}\right) \geqslant 0$, i.e. in the closure of the positive Weyl chamber,

- $0<s_{1}<1 / 2$ and $s_{2}=1 / 2$.

Proof. By the discussion preceding this Proposition, in the notation of the general overview,

$$
\begin{aligned}
\tau_{v} & = \begin{cases}\chi_{1, v} \otimes \chi_{1, v} \otimes \pi_{2, v}, & \text { if } \pi_{2, v} \text { is tempered, } \\
\chi_{1, v} \otimes \chi_{1, v} \otimes \mu_{v} \otimes \mu_{v}, & \text { otherwise, }\end{cases} \\
\underline{s}^{\prime} & = \begin{cases}(-1 / 2,1 / 2,0), & \text { if } \pi_{2, v} \text { is tempered, } \\
(-1 / 2,1 / 2, r,-r), & \text { otherwise. }\end{cases}
\end{aligned}
$$

Then, $w^{\prime}=(1,2)(3)(4)$ in the notation of the proof of the previous Proposition. Hence,

$$
\underline{s}+w^{\prime-1}\left(\underline{s}^{\prime}\right)= \begin{cases}\left(s_{1}+1 / 2, s_{1}-1 / 2, s_{2}\right), & \text { if } \pi_{2, v} \text { is tempered, } \\ \left(s_{1}+1 / 2, s_{1}-1 / 2, s_{2}+r, s_{2}-r\right), & \text { otherwise. }\end{cases}
$$


For $\underline{s}$ in the closure of the positive Weyl chamber the inequalities of Proposition 1.6 are satisfied for every $w \in W\left(M_{0}^{\prime}\right)$ since $0<r<1 / 2$. In the tempered case they are also satisfied for $0<s_{1}<1 / 2$ and $s_{2}=1 / 2$. Hence, the general overview implies the holomorphy and non-vanishing of $N\left(\underline{s}, \pi_{v}, w\right)$. For $\pi_{2, v}$ non-tempered and $0<s_{1}<1 / 2$ and $s_{2}=1 / 2$ the problem occurs if

$$
\left(s_{1}-1 / 2\right)-\left(s_{2}+r\right)=\leqslant-1 .
$$

However, repeating the same argument as in the proof of Proposition 1.12 for $\operatorname{Re}\left(s_{1}\right)=\operatorname{Re}\left(s_{2}\right)>0$ shows that $N\left(\underline{s}, \pi_{v}, w\right)$ is holomorphic and non-vanishing as claimed.

1.3. Non-split case. In this Subsection the normalizing factors are given and the holomorphy and non-vanishing of the normalized intertwining operators in the closure of the positive Weyl chamber is proved for the irreducible unitary representations $\pi_{v}^{\prime}$ of the Levi factor $M_{0}^{\prime}\left(k_{v}\right)$ of $G_{2}^{\prime}\left(k_{v}\right)$ at the places $v$ of $k$ where $D$ is non-split. We assume that $D$ splits at every archimedean place, i.e. here $v$ is non-archimedean. Since $M_{0}^{\prime}\left(k_{v}\right)$ has no proper parabolic subgroups, the representation $\pi_{v}^{\prime}$ is supercuspidal.

In order to prove required result for the minimal parabolic subgroup with the Levi factor $M_{0}^{\prime} \subset G_{2}^{\prime}$, we must consider maximal proper parabolic subgroup cases with the Levi factors $\mathrm{GL}_{1}^{\prime} \subset G_{1}^{\prime}$ and $\mathrm{GL}_{1}^{\prime} \times \mathrm{GL}_{1}^{\prime} \subset \mathrm{GL}_{2}^{\prime}$. To make the notation uniform in all three cases let $G^{\prime}$ denote one of the groups $G_{2}^{\prime}, G_{1}^{\prime}, \mathrm{GL}_{2}^{\prime}$ and $P^{\prime}=M^{\prime} N^{\prime}$ the standard parabolic subgroup with the Levi factor $M^{\prime}$, one of the Levi factors $M_{0}^{\prime}, \mathrm{GL}_{1}^{\prime}, \mathrm{GL}_{1}^{\prime} \times \mathrm{GL}_{1}^{\prime}$, respectively. The corresponding split groups are denoted by $G$ and $P=M N$.

For the Levi factor $M^{\prime}$ let $X^{*}\left(M^{\prime}\right)$ be the $\mathbb{Z}$-module of $k_{v}$-rational characters of $M^{\prime}$. As in the split case introduce the vector spaces $\mathfrak{a}_{M^{\prime}, \mathbb{C}}, \mathfrak{a}_{M^{\prime}, \mathbb{C}}^{*}$ and the homomorphism $H_{P^{\prime}}: M^{\prime} \rightarrow \mathfrak{a}_{M^{\prime}}$. At a non-split place $v$ let $\nu$ denote the absolute value of the reduced norm on $\mathrm{GL}_{1}^{\prime}\left(k_{v}\right) \cong D_{v}^{\times}$. For the split group $\mathrm{GL}_{2}$ let $\nu$ denote the absolute value of the determinant on $\mathrm{GL}_{2}\left(k_{v}\right)$. The vector space $\mathfrak{a}_{M^{\prime}, \mathbb{C}}^{*}$ is isomorphic to the split case $\mathfrak{a}_{M, \mathbb{C}}^{*}$ and we fix the isomorphism identifying $\nu$ for the split and non-split group. Moreover, we fix the isomorphism with $\mathbb{C}^{m}$ by identifying $s \in \mathbb{C}$ with $\nu^{s}$. Observe that this isomorphism for a split group might not coincide with the isomorphism used in the generic split maximal parabolic case where we identified $s \in \mathbb{C}$ with $s \widetilde{\alpha}$. In order to distinguish between the isomorphisms we will write $s$ to denote the new one and $s \widetilde{\alpha}$ to denote the old one. Moreover, in the case $\mathrm{GL}_{1}^{\prime} \times \mathrm{GL}_{1}^{\prime} \subset \mathrm{GL}_{2}^{\prime}$, although $\mathfrak{a}_{M^{\prime}, \mathbb{C}}^{*}$ is two-dimensional, as in the split case, it is enough to consider its one-dimensional subspace of the form $(s / 2,-s / 2)$ where $s \in \mathbb{C}$.

For $\underline{s} \in \mathfrak{a}_{M^{\prime}, \mathbb{C}}^{*}$ and an irreducible admissible representation $\pi_{v}^{\prime}$ of $M^{\prime}\left(k_{v}\right)$ we define the induced representation

$$
I\left(\underline{s}, \pi_{v}^{\prime}\right)=\operatorname{Ind}_{M^{\prime}\left(k_{v}\right)}^{G^{\prime}\left(k_{v}\right)}\left(\pi_{v}^{\prime} \otimes \exp \left\langle\underline{s}, H_{P^{\prime}}(\cdot)\right\rangle\right),
$$


and for $w \in W\left(M^{\prime}\right)$ the intertwining operator

$$
A\left(\underline{s}, \pi_{v}^{\prime}, w\right) f_{\underline{s}, v}(g)=\int_{U^{\prime}\left(k_{v}\right) \cap w \bar{N}^{\prime}\left(k_{v}\right) w^{-1}} f_{\underline{s}, v}\left(w^{-1} n^{\prime} g\right) d n^{\prime},
$$

where $f_{s, v}$ is in the space of the induced representation $I\left(\underline{s}, \pi_{v}^{\prime}\right), \bar{N}^{\prime}$ the unipotent radical of the opposite parabolic subgroup of $P^{\prime}$ and $U^{\prime}$ the unipotent radical of the Borel subgroup of $G^{\prime}$. The Haar measure $d n^{\prime}$ is chosen compatibly with the measure on the split form as explained in Section 2 of [42]. The dependency of $f_{\underline{s}, v}$ on $\underline{s}$ is obtained using the compact picture with respect to the fixed maximal compact subgroup as in Section II.1 of [38]. The decomposition of Proposition 1.4 holds in this case because it holds for any reductive group.

Observe that for $M^{\prime} \cong \mathrm{GL}_{1}^{\prime} \times \mathrm{GL}_{1}^{\prime}$ if we write $\pi_{v}^{\prime} \cong \pi_{1, v}^{\prime} \otimes \pi_{2, v}^{\prime}$ and $\underline{s}=\left(s_{1}, s_{2}\right) \in \mathfrak{a}_{M^{\prime}, \mathbb{C}}^{*}$, then

$$
I\left(\underline{s}, \pi_{v}^{\prime}\right)=\operatorname{Ind}_{\mathrm{GL}_{1}^{\prime}\left(k_{v}\right) \times \mathrm{GL}_{1}^{\prime}\left(k_{v}\right)}^{G^{\prime}\left(k_{v}\right)}\left(\pi_{1, v}^{\prime} \nu^{s_{1}} \otimes \pi_{2, v}^{\prime} \nu^{s_{2}}\right),
$$

where $G^{\prime}$ is $G_{2}^{\prime}$ or $\mathrm{GL}_{2}^{\prime}$. In the latter case, tensoring by the appropriate power of the absolute value of the reduced norm, shows

$$
I\left(\left(s_{1}, s_{2}\right), \pi_{v}^{\prime}\right) \cong I\left(\left(\left(s_{1}-s_{2}\right) / 2,-\left(s_{1}-s_{2}\right) / 2\right), \pi_{v}^{\prime}\right) .
$$

Observe that for the local lift $\pi_{v}$ of $\pi_{v}^{\prime}$ the $\mathrm{GL}_{4}\left(k_{v}\right)$ induced representation

$$
I\left(\left(s_{1}, s_{2}\right), \pi_{v}\right) \cong I\left(\left(\left(s_{1}-s_{2}\right) / 2,-\left(s_{1}-s_{2}\right) / 2\right), \pi_{v}\right)=I\left(\left(s_{1}-s_{2}\right) \widetilde{\alpha}, \pi_{v}\right) .
$$

For $M^{\prime} \cong \mathrm{GL}_{1}^{\prime}$ if $s \in \mathfrak{a}_{M^{\prime}, \mathbb{C}}^{*}$ then

$$
I\left(s, \pi_{v}^{\prime}\right)=\operatorname{Ind}_{\mathrm{GL}_{1}^{\prime}\left(k_{v}\right)}^{G_{1}^{\prime}\left(k_{v}\right)}\left(\pi_{v}^{\prime} \nu^{s}\right) .
$$

Observe that for the local lift $\pi_{v}$ of $\pi_{v}^{\prime}$ the induced representation

$$
I\left(s, \pi_{v}\right) \cong \operatorname{Ind}_{\mathrm{GL}_{2}\left(k_{v}\right)}^{\mathrm{SO}_{4}\left(k_{v}\right)}\left(\pi_{v} \nu^{s}\right) \cong I\left(2 s \widetilde{\alpha}, \pi_{v}\right) .
$$

The normalization of the intertwining operators in the non-split case is defined using the local lift of representations from $\mathrm{GL}_{1}^{\prime}\left(k_{v}\right) \cong D_{v}^{\times}$to the split $\mathrm{GL}_{2}\left(k_{v}\right)$. Every irreducible representation $\pi_{v}^{\prime}$ of $M^{\prime}\left(k_{v}\right)$ is supercuspidal because $M^{\prime}\left(k_{v}\right)$ has no proper parabolic subgroups. Let $\pi_{v}$ denote its local lift to $M\left(k_{v}\right)$. It is always square-integrable. Then, the normalizing factor for the intertwining operator $A\left(\underline{s}, \pi_{v}^{\prime}, w\right)$ is defined to be

$$
r\left(\underline{s}, \pi_{v}^{\prime}, w\right)=r\left(\underline{s}, \pi_{v}, w\right)
$$

normalized intertwining operator

$$
N\left(\underline{s}, \pi_{v}^{\prime}, w\right)=r\left(\underline{s}, \pi_{v}^{\prime}, w\right)^{-1} A\left(\underline{s}, \pi_{v}^{\prime}, w\right) .
$$


More precisely, for the maximal parabolic case $\mathrm{GL}_{1}^{\prime} \subset G_{1}^{\prime}$, the adjoint representation $r$ is the exterior square of the standard representation of $\mathrm{GL}_{2}(\mathbb{C})$ and the normalizing factor is

$$
r\left(s, \pi_{v}^{\prime}, w_{2}\right)=r\left(2 s \widetilde{\alpha}, \pi_{v}, w_{2}\right)=\frac{L\left(2 s, \omega_{\pi_{v}}\right)}{L\left(1+2 s, \omega_{\pi_{v}}\right) \varepsilon\left(2 s, \omega_{\pi_{v}}, \psi_{v}\right)},
$$

where $w_{2}$ is the unique nontrivial element of the Weyl group of this case. For the maximal parabolic case $\mathrm{GL}_{1}^{\prime}\left(k_{v}\right) \times \mathrm{GL}_{1}^{\prime}\left(k_{v}\right) \subset \mathrm{GL}_{2}^{\prime}\left(k_{v}\right)$, the adjoint representation $r$ is the tensor product of two standard representations of $\mathrm{GL}_{2}(\mathbb{C})$ and

$$
\begin{aligned}
r\left(\left(s_{1}, s_{2}\right), \pi_{v}^{\prime}, w_{1}\right) & =r\left(\left(s_{1}-s_{2}\right) \widetilde{\alpha}, \pi_{v}, w_{1}\right) \\
(1.18) & =\frac{L\left(s_{1}-s_{2}, \pi_{1, v} \times \widetilde{\pi}_{2, v}\right)}{L\left(1+s_{1}-s_{2}, \pi_{1, v} \times \widetilde{\pi}_{2, v}\right) \varepsilon\left(s_{1}-s_{2}, \pi_{1, v} \times \widetilde{\pi}_{2, v}, \psi_{v}\right)}
\end{aligned}
$$

where $w_{1}$ is the unique nontrivial element of the Weyl group of this case.

As in the generic split case, the proof of holomorphy and non-vanishing starts with the maximal parabolic subgroup cases and then uses the decomposition of Proposition 1.4. But first we need the equality of the Plancherel measures proved in the Siegel cases $\mathrm{GL}_{n}^{\prime} \subset G_{n}^{\prime}$ in Proposition 2.1 of [42]. The case of $\mathrm{GL}_{1}^{\prime} \times \mathrm{GL}_{1}^{\prime} \subset \mathrm{GL}_{2}^{\prime}$ is settled in the same way.

LEMMA 1.19. Let $M^{\prime} \subset G^{\prime}$ be one of the maximal parabolic subgroup cases considered, i.e. $\mathrm{GL}_{1}^{\prime} \subset G_{1}^{\prime}$ or $\mathrm{GL}_{1}^{\prime} \times \mathrm{GL}_{1}^{\prime} \subset \mathrm{GL}_{2}^{\prime}$. Let $\pi_{v}^{\prime}$ be an irreducible unitary representation of $M^{\prime}\left(k_{v}\right)$ and $\pi_{v}$ its local lift to $M\left(k_{v}\right)$. Then, the Plancherel measures of $\pi_{v}^{\prime}$ and $\pi_{v}$ are equal, i.e. $\mu\left(s, \pi_{v}^{\prime}\right)=\mu\left(s, \pi_{v}\right)$ for every $s \in \mathfrak{a}_{M^{\prime}, \mathbb{C}}^{*}$, where $s$ denotes $(s / 2,-s / 2)$ in the case $\mathrm{GL}_{1}^{\prime} \times \mathrm{GL}_{1}^{\prime} \subset \mathrm{GL}_{2}^{\prime}$. The measures on the unipotent radicals $N^{\prime}\left(k_{v}\right)$ and $N\left(k_{v}\right)$ used to define the intertwining operators are chosen to be compatible as in Section 2 of [42].

REMARK 1.20. Since $\pi_{v}$ is square-integrable, it is a generic representation of $M\left(k_{v}\right)$. The adjoint representation $r$ is irreducible. Thus, by equations (3.6) and (7.4) of [45] the Plancherel measure of $\pi_{v}$ equals

$$
\begin{aligned}
\mu\left(s \widetilde{\alpha}, \pi_{v}\right) & =\frac{\varepsilon\left(s, \pi_{v}, r, \psi_{v}\right) L\left(1-s, w\left(\pi_{v}\right), r\right)}{L\left(s, \pi_{v}, r\right)} \frac{\varepsilon\left(-s, w\left(\pi_{v}\right), r, \psi_{v}\right) L\left(1+s, \pi_{v}, r\right)}{L\left(-s, w\left(\pi_{v}\right), r\right)} \\
& =r\left(s \widetilde{\alpha}, \pi_{v}, w\right)^{-1} r\left(-s \widetilde{\alpha}, w\left(\pi_{v}\right), w^{-1}\right)^{-1} .
\end{aligned}
$$

By the previous Lemma

$$
\mu\left(s, \pi_{v}^{\prime}\right)=\mu\left(s, \pi_{v}\right)=r\left(s, \pi_{v}, w\right)^{-1} r\left(-s, w\left(\pi_{v}\right), w^{-1}\right)^{-1},
$$

which verifies equation (4.1) of [1] for the normalizing factors. Therefore, by the remark in Section 4 of [1], the normalization factors defined here satisfy Theorem 2.1 of [1] and we will use it in the sequel. See also Section 2.2 of [5].

Proposition 1.21. Let $M^{\prime} \subset G^{\prime}$ be one of the maximal parabolic subgroup cases considered, i.e. $\mathrm{GL}_{1}^{\prime} \subset G_{1}^{\prime}$ or $\mathrm{GL}_{1}^{\prime} \times \mathrm{GL}_{1}^{\prime} \subset \mathrm{GL}_{2}^{\prime}$. Let $\pi_{v}^{\prime}$ be an 
irreducible unitary representation of $M^{\prime}\left(k_{v}\right)$. Then, for $\operatorname{Re}(s) \geqslant 0$ the normalized intertwining operator $N\left(s, \pi_{v}^{\prime}, w\right)$ is holomorphic and non-vanishing, while for $\operatorname{Re}(s)<0$ it is holomorphic and non-vanishing if the induced representation $I\left(-s, \pi_{v}^{\prime}\right)$ is irreducible.

Proof. For $\operatorname{Re}(s)>0$, by the Langlands classification, the intertwining operator $A\left(s, \pi_{v}^{\prime}, w\right)$ is holomorphic and non-vanishing since $\pi_{v}^{\prime}$ is supercuspidal. The $\mathrm{L}$-functions appearing in $r\left(s, \pi_{v}, w\right)$ are also holomorphic and nonvanishing for $\operatorname{Re}(s)>0$ by Section 3 of [1] for the archimedean and Section 4 of [7] for the non-archimedean case since the lift $\pi_{v}$ is square-integrable. Hence, the normalized intertwining operator $N\left(s, \pi_{v}^{\prime}, w\right)$ is holomorphic and non-vanishing for $\operatorname{Re}(s)>0$.

For $\operatorname{Re}(s)=0$, by the definition of the Plancherel measure, Lemma 1.19 and Remark 1.20

$$
\mu\left(s, \pi_{v}^{\prime}\right)^{-1}=A\left(-s, w\left(\pi_{v}^{\prime}\right), w^{-1}\right) A\left(s, \pi_{v}^{\prime}, w\right)=r\left(s, \pi_{v}, w\right) r\left(-s, w\left(\pi_{v}\right), w^{-1}\right) .
$$

Hence,

$$
N\left(-s, w\left(\pi_{v}^{\prime}\right), w^{-1}\right) N\left(s, \pi_{v}^{\prime}, w\right)=I d .
$$

By Remark 1.20, Theorem 2.1 of [1] holds and specially

$$
N\left(s, \pi_{v}^{\prime}, w\right)^{*}=N\left(-s, w\left(\pi_{v}^{\prime}\right), w^{-1}\right) .
$$

Since the representations are admissible, the space of $K^{\prime}$-invariants for every open compact subgroup $K^{\prime}$ of $G^{\prime}\left(k_{v}\right)$ is finite-dimensional and $N\left(s, \pi_{v}^{\prime}, w\right)$ restricted to any such finite-dimensional space is a unitary operator. Therefore, it is bounded and its operator norm is at most one. Taking $K^{\prime}$ smaller and smaller, the holomorphy follows. The non-vanishing is a consequence of holomorphy by Zhang's Lemma (Lemma 1.7 of [25]).

For $\operatorname{Re}(s)<0$, note that $\operatorname{Re}(-s)>0$ and hence $N\left(-s, w\left(\pi_{v}^{\prime}\right), w^{-1}\right)$ is holomorphic and non-vanishing. If the induced representation $I\left(-s, w\left(\pi_{v}^{\prime}\right)\right)$ is irreducible, then $N\left(-s, w\left(\pi_{v}^{\prime}\right), w^{-1}\right)$ is an isomorphism. Now

$$
N\left(-s, w\left(\pi_{v}^{\prime}\right), w^{-1}\right) N\left(s, \pi_{v}^{\prime}, w\right)=I d
$$

implies that $N\left(s, \pi_{v}^{\prime}, w\right)$ is also an isomorphism and hence holomorphic and non-vanishing.

Proposition 1.22. Let $\pi_{v}^{\prime} \cong \pi_{1, v}^{\prime} \otimes \pi_{2, v}^{\prime}$ be an irreducible unitary representation of the Levi factor $M_{0}^{\prime}\left(k_{v}\right) \cong \mathrm{GL}_{1}^{\prime}\left(k_{v}\right) \times \mathrm{GL}_{1}^{\prime}\left(k_{v}\right)$ of $G_{2}^{\prime}\left(k_{v}\right)$ and $\underline{s}=\left(s_{1}, s_{2}\right) \in \mathfrak{a}_{M_{0}^{\prime}, \mathbb{C}}^{*}$. Then, for every $w \in W$, the normalized intertwining operator $N\left(\underline{s}, \pi_{v}^{\prime}, w\right)$ is holomorphic and non-vanishing for $\underline{s}=\left(s_{1}, s_{2}\right)$ such that

- $\operatorname{Re}\left(s_{1}\right) \geqslant \operatorname{Re}\left(s_{2}\right) \geqslant 0$, i.e. in the closure of the positive Weyl chamber,

- $0<s_{1}<1 / 2$ and $s_{2}=1 / 2$,

- $1 / 2<s_{1}<1$ and $s_{1}-s_{2}=1$,

- $1<s_{1}<2$ and $s_{1}-s_{2}=2$ except $\left(s_{1}, s_{2}\right)=(3 / 2,-1 / 2)$. 
At the exceptional point $\left(s_{1}, s_{2}\right)=(3 / 2,-1 / 2)$ it is always holomorphic and non-vanishing for $w \in\left\{1, w_{1}, w_{2} w_{1}, w_{1} w_{2} w_{1}\right\}$. Moreover, for $w \in$ $\left\{w_{2}, w_{1} w_{2}, w_{2} w_{1} w_{2}, w_{1} w_{2} w_{1} w_{2}\right\}$, it is holomorphic and non-vanishing at $\left(s_{1}, s_{2}\right)=(3 / 2,-1 / 2)$ if the central character $\omega_{\pi_{2, v}^{\prime}}$ is nontrivial.

Proof. For holomorphy write the intertwining operator $N\left(\underline{s}, \pi_{v}^{\prime}, w\right)$ as the composition of the intertwining operators for the maximal parabolic cases as in Proposition 1.4. Since $\pi_{v}^{\prime}$ is supercuspidal, by Proposition 1.22, the maximal parabolic case normalized intertwining operators are holomorphic for $\underline{s}=\left(s_{1}, s_{2}\right)$ satisfying the conditions of the Proposition. By [42] the condition on the central character assures that the induced representation $I\left(-1 / 2, \pi_{2, v}^{\prime}\right)$ is irreducible as required in Proposition 1.22. The point $\left(s_{1}, s_{2}\right)=(3 / 2,-1 / 2)$ is excluded in the last open interval if the induced representation $I\left(-1 / 2, \pi_{2, v}^{\prime}\right)$ is reducible because in that case the $G_{1}^{\prime}\left(k_{v}\right)$ normalized intertwining operator $N\left(-1 / 2, \pi_{2, v}^{\prime}, w_{2}\right)$ is not covered by the previous Proposition. For the particular Weyl group elements in the last claim, the intertwining operator $N\left(-1 / 2, \pi_{2, v}^{\prime}, w_{2}\right)$ does not occur in the decomposition. The non-vanishing follows from the holomorphy by Zhang's Lemma (Lemma 1.7 of [25]).

\section{Calculation of the Residual spectrum}

The calculation of the residual spectrum in this paper relies on the Langlands spectral theory as explained in [30] and [38]. See also [33] and [34]. However, dealing with the low rank group $G_{2}^{\prime}$ allows us to follow the exposition of [22] rather closely.

2.1. Brief Overview of the Method. Let $L^{2}\left(G_{2}^{\prime}\right)$ be the space of automorphic forms on $G_{2}^{\prime}(\mathbb{A})$ as defined in Section I.2.17 of [38]. Then, the first step in the decomposition of $L^{2}\left(G_{2}^{\prime}\right)$ is the decomposition according to the cuspidal data given in Section II.2.4 of [38]. Since in $G_{2}^{\prime}$ there are three standard proper parabolic subgroups, all being self-associate, the decomposition according to the cuspidal data for the group $G_{2}^{\prime}(\mathbb{A})$ gives the decomposition into the Hilbert space direct sum

$$
L^{2}\left(G_{2}^{\prime}\right) \cong L_{M_{0}^{\prime}}^{2} \oplus L_{M_{1}^{\prime}}^{2} \oplus L_{M_{2}^{\prime}}^{2},
$$

where $L_{M_{i}^{\prime}}^{2}$ decomposes further into

$$
L_{M_{i}^{\prime}}^{2} \cong \oplus_{\pi^{\prime}} L_{\left(M_{i}^{\prime}, \pi^{\prime}\right)}^{2},
$$

where the sum is over all the cuspidal automorphic representations $\pi^{\prime}$ of $M_{i}^{\prime}(\mathbb{A})$. For a cuspidal automorphic representation $\pi^{\prime}$ of $M_{i}^{\prime}(\mathbb{A})$, the space $L_{\left(M_{i}^{\prime}, \pi^{\prime}\right)}^{2}$ is described using the Eisenstein series attached to $\pi^{\prime}$ as in Section II.1.12 of [38]. We recall the description below. In this paper we consider the residual part of the space $L_{M_{0}^{\prime}}^{2}$, where $M_{0}^{\prime} \cong \mathrm{GL}_{1}^{\prime} \times \mathrm{GL}_{1}^{\prime}$ is the Levi factor of the minimal standard parabolic subgroup $P_{0}^{\prime}$ of $G_{2}^{\prime}$. 
The space $\mathfrak{a}_{M_{0}^{\prime}, \mathbb{C}}^{*}$ is two-dimensional and we take $\nu$, the absolute value of the reduced norm, on every factor of $M_{0}^{\prime}$ isomorphic to $\mathrm{GL}_{1}^{\prime}$ as the basis elements. Then $\underline{s}=\left(s_{1}, s_{2}\right) \in \mathbb{C}^{2}$ corresponds to the character $\left(\nu^{s_{1}}, \nu^{s_{2}}\right)$ of $M_{0}^{\prime}$. The positive Weyl chamber is given by $\operatorname{Re}\left(s_{1}\right)>\operatorname{Re}\left(s_{2}\right)>0$.

Denote by $w_{1}$ and $w_{2}$ the reflections with respect to simple roots $e_{1}-$ $e_{2}$ and $2 e_{2}$ of $G_{2}^{\prime}$. These elements of $W\left(M_{0}^{\prime}\right)$ correspond to the maximal parabolic case intertwining operators for $\mathrm{GL}_{1}^{\prime} \times \mathrm{GL}_{1}^{\prime} \subset \mathrm{GL}_{2}^{\prime}$ and $\mathrm{GL}_{1}^{\prime} \subset G_{1}^{\prime}$, respectively. Then

$$
W\left(M_{0}^{\prime}\right)=\left\{1, w_{1}, w_{2}, w_{1} w_{2}, w_{2} w_{1}, w_{1} w_{2} w_{1}, w_{2} w_{1} w_{2}, w_{1} w_{2} w_{1} w_{2}\right\} .
$$

In the following Table 1 the action of the elements of $W\left(M_{0}^{\prime}\right)$ on $\underline{s}=\left(s_{1}, s_{2}\right) \in$ $\mathfrak{a}_{M_{0}^{\prime}, \mathbb{C}}$ and a cuspidal automorphic representation $\pi^{\prime}=\otimes_{v} \pi_{v}^{\prime} \cong \pi_{1}^{\prime} \otimes \pi_{2}^{\prime}=$ $\left(\otimes_{v} \pi_{1, v}^{\prime}\right) \otimes\left(\otimes_{v} \pi_{2, v}^{\prime}\right)$ of the Levi factor $M_{0}^{\prime}(\mathbb{A}) \cong \mathrm{GL}_{1}^{\prime}(\mathbb{A}) \times \mathrm{GL}_{1}^{\prime}(\mathbb{A})$ is given. Here $\sim$ denotes the contragredient representation.

\begin{tabular}{|c|c|c|}
\hline$w$ & $w(\underline{s})=w\left(s_{1}, s_{2}\right)$ & $w\left(\pi^{\prime}\right) \cong w\left(\pi_{1}^{\prime} \otimes \pi_{2}^{\prime}\right)$ \\
\hline \hline 1 & $\left(s_{1}, s_{2}\right)$ & $\pi_{1}^{\prime} \otimes \pi_{2}^{\prime}$ \\
\hline$w_{1}$ & $\left(s_{2}, s_{1}\right)$ & $\pi_{2}^{\prime} \otimes \pi_{1}^{\prime}$ \\
\hline$w_{2}$ & $\left(s_{1},-s_{2}\right)$ & $\pi_{1}^{\prime} \otimes \widetilde{\pi}_{2}^{\prime}$ \\
\hline$w_{1} w_{2}$ & $\left(-s_{2}, s_{1}\right)$ & $\widetilde{\pi}_{2}^{\prime} \otimes \pi_{1}^{\prime}$ \\
\hline$w_{2} w_{1}$ & $\left(s_{2},-s_{1}\right)$ & $\pi_{2}^{\prime} \otimes \widetilde{\pi}_{1}^{\prime}$ \\
\hline$w_{1} w_{2} w_{1}$ & $\left(-s_{1}, s_{2}\right)$ & $\widetilde{\pi}_{1}^{\prime} \otimes \pi_{2}^{\prime}$ \\
\hline$w_{2} w_{1} w_{2}$ & $\left(-s_{2},-s_{1}\right)$ & $\widetilde{\pi}_{2}^{\prime} \otimes \widetilde{\pi}_{1}^{\prime}$ \\
\hline$w_{1} w_{2} w_{1} w_{2}$ & $\left(-s_{1},-s_{2}\right)$ & $\widetilde{\pi}_{1}^{\prime} \otimes \widetilde{\pi}_{2}^{\prime}$ \\
\hline
\end{tabular}

TABLE 1 . Action of $W\left(M_{0}^{\prime}\right)$

Let $\pi^{\prime} \cong \otimes_{v} \pi_{v}^{\prime}$ be a cuspidal automorphic representation of $M_{0}^{\prime}(\mathbb{A})$. For $\underline{s}=\left(s_{1}, s_{2}\right) \in \mathfrak{a}_{M_{0}^{\prime}, \mathbb{C}}^{*}$ we form the induced representation

$$
I\left(\underline{s}, \pi^{\prime}\right)=\operatorname{Ind}_{M_{0}^{\prime}(\mathbb{A})}^{G^{\prime}(\mathbb{A})}\left(\pi^{\prime} \otimes \exp \left\langle\underline{s}, H_{P_{0}^{\prime}}(\cdot)\right\rangle\right),
$$

where $H_{P_{0}^{\prime}}: M_{0}^{\prime} \rightarrow \mathfrak{a}_{M_{0}^{\prime}}$ is the homomorphism defined in Section 1. It is the restricted tensor product of the local induced representations $I\left(\underline{s}, \pi^{\prime}\right) \cong$ $\otimes_{v} I\left(\underline{s}, \pi_{v}^{\prime}\right)$, where the local induced representations $I\left(\underline{s}, \pi_{v}^{\prime}\right)$ are defined in the previous Section. At almost all places, $I\left(\underline{s}, \pi_{v}^{\prime}\right)$ is unramified and the tensor product is restricted with respect to the suitably normalized unramified functions $f_{\underline{s}, v}^{\circ}$ at those places, i.e. the functions invariant for the fixed maximal compact subgroup normalized by $f_{\underline{s}, v}^{\circ}\left(I_{v}\right)=1$, where $I_{v}$ is the identity matrix. The induced representation $I\left(\underline{s}, \pi^{\prime}\right)$ is always identified with its realization in the space of automorphic forms. The sections $f_{\underline{s}} \in I\left(\underline{s}, \pi^{\prime}\right)$ of automorphic forms are always chosen in such a way that the function $\underline{s} \mapsto f_{\underline{s}}$ 
is analytic on $\mathfrak{a}_{M_{0}^{\prime}, \mathbb{C}}^{*}$ and Paley-Wiener with values in the space of the induced representations as explained in Sections II.1.1 and II.1.2 of [38].

As in Section II.1.6 of [38] we define, for $w \in W\left(M_{0}^{\prime}\right)$ and $f_{\underline{s}} \in I\left(\underline{s}, \pi^{\prime}\right)$, the standard intertwining operator as the analytic continuation from the domain of convergence of the integral

$$
A\left(\underline{s}, \pi^{\prime}, w\right) f_{\underline{s}}(g)=\int_{U^{\prime}(\mathbb{A}) \cap w \bar{N}_{0}^{\prime}(\mathbb{A}) w^{-1}} f_{\underline{s}}\left(w^{-1} n^{\prime} g\right) d n^{\prime},
$$

where $U^{\prime}$ is the unipotent radical of the Borel subgroup in $G_{2}^{\prime}$ and $\bar{N}_{0}^{\prime}$ is the unipotent radical of the opposite parabolic subgroup of $P_{0}^{\prime}$. The fixed Haar measure $d n^{\prime}$ is chosen as in Section 2 of [41]. Away from the poles it is an intertwining operator from $I\left(\underline{s}, \pi^{\prime}\right)$ to $I\left(w(\underline{s}), w\left(\pi^{\prime}\right)\right)$. Here $w(\underline{s})$ and $w\left(\pi^{\prime}\right)$ denotes the action of the Weyl group induced by the conjugation on the Levi factor $M_{0}^{\prime}$. The intertwining operator decomposes into the restricted tensor product of the local intertwining operators defined in the previous Section, i.e. if $f_{\underline{s}}=\otimes_{v} f_{\underline{s}, v}$, then

$$
A\left(\underline{s}, \pi^{\prime}, w\right) f_{\underline{s}}=\otimes_{v} A\left(\underline{s}, \pi_{v}^{\prime}, w\right) f_{\underline{s}, v} .
$$

Here $f_{\underline{s}, v}=f_{\underline{s}, v}^{\circ}$ at almost all places. At those places

$$
A\left(\underline{s}, \pi_{v}^{\prime}, w\right) f_{\underline{s}, v}^{\circ}=r\left(\underline{s}, \pi_{v}^{\prime}, w\right) \tilde{f}_{w(\underline{s}), v}^{\circ},
$$

where $\tilde{f}_{w(\underline{s}), v}^{\circ}$ is unramified in $I\left(w(\underline{s}), w\left(\pi_{v}^{\prime}\right)\right)$ normalized by $\widetilde{f}_{w(\underline{s}), v}^{\circ}\left(I_{v}\right)=1$ and $r\left(\underline{s}, \pi_{v}^{\prime}, w\right)$ is the normalizing factor.

As in Section II.1.5 of [38] we define, for $\underline{s} \in \mathfrak{a}_{M_{0}^{\prime}, \mathbb{C}}^{*}$ and $f_{\underline{s}} \in I\left(\underline{s}, \pi^{\prime}\right)$, the Eisenstein series attached to $\pi^{\prime}$ as the analytic continuation from the domain of convergence of the following series

$$
E\left(\underline{s}, g ; f_{\underline{s}}, \pi^{\prime}\right)=\sum_{\gamma \in P_{0}^{\prime}(k) \backslash G_{2}^{\prime}(k)} f_{\underline{s}}(\gamma g),
$$

where $g \in G_{2}^{\prime}(\mathbb{A})$. It is meromorphic as a function of $\underline{s}$. By Section II.1.12 of [38], the space $L_{\left(M_{0}^{\prime}, \pi^{\prime}\right)}^{2}$ is the closure of the space generated by the functions of the form

$$
g \mapsto \frac{1}{(2 \pi i)^{2}} \int_{\operatorname{Re}(\underline{s})=\underline{s}_{0}} E\left(\underline{s}, g ; f_{\underline{s}}, \pi^{\prime}\right) d \underline{s},
$$

where $\underline{s}_{0}$ is in the domain of the absolute convergence of the integral defining the standard intertwining operators and the series defining the Eisenstein series.

In order to distinguish the residual part of the space $L_{\left(M_{0}^{\prime}, \pi^{\prime}\right)}^{2}$ we apply the residue theorem of Section V.1.5 of [38] to move the line of integration into the origin of $\mathfrak{a}_{M_{0}^{\prime}, \mathbb{C}}^{*}$, i.e. to the line $\operatorname{Re}(\underline{s})=(0,0)$. Before giving more details on the application of the residue theorem in our case, let us consider the singular hyperplanes of the Eisenstein series inside the positive Weyl chamber. Since 
it suffices to consider the real poles of the Eisenstein series as explained in the Introduction, it is enough to view the singularities of the Eisenstein series inside the real part of $\mathfrak{a}_{M_{0}^{\prime}, \mathbb{C}}$.

The analytic properties of the Eisenstein series, such as the position and order of the poles and the square integrability, coincide with the analytic properties of their constant term along $P_{0}^{\prime}$, i.e.

$$
E_{0}\left(\underline{s}, g ; f_{\underline{s}}, \pi^{\prime}\right)=\int_{N_{0}^{\prime}(k) \backslash N_{0}^{\prime}(\mathbb{A})} E\left(\underline{s}, n^{\prime} g ; f_{\underline{s}}, \pi^{\prime}\right) d n^{\prime} .
$$

On the other hand by Proposition II.1.7. of [38] the constant term equals

$$
E_{0}\left(\underline{s}, g ; f_{\underline{s}}, \pi^{\prime}\right)=\sum_{w \in W\left(M_{0}^{\prime}\right)} A\left(\underline{s}, \pi^{\prime}, w\right) f_{\underline{s}}(g) .
$$

Hence, the singularities of the Eisenstein series are the same as the singularities of the sum of the intertwining operators in (2.2). This is where the normalization of the intertwining operators of the preceding Section comes into play, enabling us to find the singularities inside the closure of the positive Weyl chamber as the singularities of the normalizing factors which are given using the $\mathrm{L}$-functions. But the normalizing factors depend on the form of the cuspidal automorphic representation $\pi^{\prime} \cong \pi_{1}^{\prime} \otimes \pi_{2}^{\prime}$. More precisely, we distinguish three cases:

CASE A Both $\pi_{1}$ and $\pi_{2}$ are cuspidal automorphic representations,

CASE B One of $\pi_{1}$ and $\pi_{2}$ is a cuspidal and the other is a residual automorphic representation,

CASE C Both $\pi_{1}$ and $\pi_{2}$ are residual automorphic representations.

In the following Theorem we restate the results of the preceding Section in the global context. The standard proof is omitted.

THEOREM 2.3. Let $\pi^{\prime}=\otimes_{v} \pi_{v}^{\prime}$ be a cuspidal automorphic representation of the group $M_{0}^{\prime}(\mathbb{A})$. Then, for every $w \in W\left(M_{0}^{\prime}\right)$, the global normalizing factor

$$
r\left(\underline{s}, \pi^{\prime}, w\right)=\prod_{v} r\left(\underline{s}, \pi_{v}^{\prime}, w\right)
$$

is a meromorphic function of $\underline{s}$, and for every $w \in W\left(M_{0}^{\prime}\right)$ the global normalized operator

$$
N\left(\underline{s}, \pi^{\prime}, w\right)=r\left(\underline{s}, \pi^{\prime}, w\right)^{-1} A\left(\underline{s}, \pi^{\prime}, w\right)
$$

is holomorphic and non-vanishing for $\underline{s}=\left(s_{1}, s_{2}\right)$ such that

- $\operatorname{Re}\left(s_{1}\right) \geqslant \operatorname{Re}\left(s_{2}\right) \geqslant 0$, except at $\underline{s}=(0,0)$ in case $C$, i.e. in the closure of the positive Weyl chamber, except the origin in case $C$,

- $0<s_{1}<1 / 2$ and $s_{2}=1 / 2$,

- In case $A, 1 / 2<s_{1}<1$ and $s_{1}-s_{2}=1$,

- In case $C, 1<s_{1}<2$ and $s_{1}-s_{2}=2$ except $\left(s_{1}, s_{2}\right)=(3 / 2,-1 / 2)$. 
In case $C$, at the exceptional point $\left(s_{1}, s_{2}\right)=(3 / 2,-1 / 2)$ it is always holomorphic and non-vanishing for $w \in\left\{1, w_{1}, w_{2} w_{1}, w_{1} w_{2} w_{1}\right\}$.

REMARK 2.4. The behavior of the global intertwining operator at $(3 / 2,-1 / 2)$ in case $C$ for $w=w_{1} w_{2} w_{1} w_{2}$ is also required in the calculation. However, we will study that behavior during the calculation of the residual spectrum at that point in case $\mathrm{C}$.

Now, inside the closure of the positive Weyl chamber, the singular hyperplanes of the normalizing factors $r\left(\underline{s}, \pi^{\prime}, w\right)$ for $w \in W\left(M_{0}^{\prime}\right)$ are in fact the possible singular hyperplanes of the Eisenstein series $E\left(\underline{s}, g ; f_{\underline{s}}, \pi^{\prime}\right)$. The possible singular hyperplanes in each of the three cases are given in Figures 1, 2 and 3 below, where only the real part of $\mathfrak{a}_{M_{0}^{\prime}, \mathbb{C}}^{*}$ is presented. The Eisenstein series is a meromorphic function with the (possible) polynomial singularities along these hyperplanes in a sense of Section V.1.3 of [38].

Finally, we explain how to apply the residue theorem of Section V.1.5 of [38] to decompose the residual part of the space $L_{\left(M_{0}^{\prime}, \pi^{\prime}\right)}^{2}$ for a cuspidal automorphic representation $\pi^{\prime}$ of $M_{0}^{\prime}(\mathbb{A})$. We choose a path connecting $\underline{s}_{0}$ and the origin of $\mathfrak{a}_{M_{0}^{\prime}, \mathbb{C}}^{*}$ satisfying the conditions of Section V.1.5 of [38] as indicated by the dashed line in Figures 1, 2 and 3. It intersects every singular hyperplane inside the positive Weyl chamber in only one real point and avoids the intersections of singular hyperplanes.

The residue theorem of Section V.1.5 of [38] and the decomposition of Section V.3.13 of [38] (see also Section 5 of [22]), show that the integral (2.1) generating the space $L_{\left(M_{0}^{\prime}, \pi^{\prime}\right)}^{2}$, after deforming the line of integration to the origin, gives

$$
g \mapsto \frac{1}{(2 \pi i)^{2}} \int_{\operatorname{Re}(\underline{s})=(0,0)} E\left(\underline{s}, g ; f_{\underline{s}}, \pi^{\prime}\right) d \underline{s}
$$

which generate a part of the continuous spectrum attached to $\pi^{\prime}$, and the sum

$$
\sum_{\mathcal{S}} \frac{1}{2 \pi i} \int_{\operatorname{Re}(\underline{s})=\underline{s}_{0, \mathcal{S}}} \operatorname{Res} \mathcal{S} E\left(\underline{s}, g ; f_{\underline{s}}, \pi^{\prime}\right) d \underline{s}
$$

of the residues of the Eisenstein series along the singular hyperplanes $\mathcal{S}$, where $\underline{s}_{0, \mathcal{S}}$ is the real point of the intersection of $\mathcal{S}$ with the path of deformation.

Next, for every singular hyperplane $\mathcal{S}$, we fix the new coordinate $z$ on $\mathcal{S}$ such that $z=0$ corresponds to the orthogonal projection of the origin of $\mathfrak{a}_{M_{0}^{\prime}, \mathbb{C}}^{*}$ on $\mathcal{S}$. Let $z_{0}$ be the new coordinate of the intersection point $\underline{s}_{0, \mathcal{S}}$. Then, the integrals in (2.5) become

$$
\int_{\operatorname{Re}(z)=z_{0}} \operatorname{Res} \mathcal{S} E\left(z, g ; f_{\underline{s}}, \pi^{\prime}\right) d z,
$$

where $\operatorname{Res}_{\mathcal{S}} E\left(z, g ; f_{\underline{s}}, \pi^{\prime}\right)$ is the residue along $\mathcal{S}$ written in the new variable $z$. By the residue theorem in one variable applied to the singular hyperplane 
$\mathcal{S}$, we deform the line of integration from $\operatorname{Re}(z)=z_{0}$ to the origin $\operatorname{Re}(z)=0$ as indicated by the dotted lines in Figures 1,2 and 3. Again, at the origin of $\mathcal{S}$ we obtain a part of the continuous spectrum, while the residues which we pick up along the way, if square-integrable (and non-zero), generate the part of the residual spectrum $L_{r e s,\left(M_{0}^{\prime}, \pi^{\prime}\right)}^{2}$ inside $L_{\left(M_{0}^{\prime}, \pi^{\prime}\right)}^{2}$. Hence, the contribution at a singular point $z=\zeta$ on $\mathcal{S}$ is the iterated residue

$$
\operatorname{Res}_{z=\zeta} \operatorname{Res}_{\mathcal{S}} E\left(\underline{s}, g ; f_{\underline{s}}, \pi^{\prime}\right) \text {. }
$$

The Langlands square-integrability criterion for the automorphic forms obtained as the iterated residues (2.6) is given in the following Lemma. For the proof see Section I.4.11 of [38] or page 104 of [30]. Recall that the analytic properties of the Eisenstein series coincide with the analytic properties of its constant term.

Lemma 2.7. Let $\pi^{\prime}$ be a cuspidal automorphic representation of $M_{0}^{\prime}(\mathbb{A})$. Let the iterated residue

$$
\operatorname{Res}_{z=\zeta} \operatorname{Res}_{\mathcal{S}} E_{0}\left(\underline{s}, g ; f_{\underline{s}}, \pi^{\prime}\right)
$$

of the constant term of the Eisenstein series attached to $\pi^{\prime}$ at a point $\underline{s}=$ $\left(s_{1}, s_{2}\right)$ be equal to

$$
\sum_{w \in W_{0}^{\prime} \subset W\left(M_{0}^{\prime}\right)} C_{w} \cdot N\left(\underline{s}, \pi^{\prime}, w\right) f_{\underline{s}}(g),
$$

where $C_{w}$ are nonzero constants. Then, the automorphic forms in the space $L_{\text {res },\left(M_{0}^{\prime}, \pi^{\prime}\right)}^{2}$ obtained as the iterated residue (2.6) are square-integrable if and only if $w(\underline{s})=\left(s_{1}^{\prime}, s_{2}^{\prime}\right)$ satisfies

$$
\begin{aligned}
s_{1}^{\prime} & <0, \\
s_{1}^{\prime}+s_{2}^{\prime} & <0,
\end{aligned}
$$

for all $w \in W_{0}^{\prime}$ such that $f_{\underline{s}}$ is not in the kernel of the normalized intertwining operator $N\left(\underline{s}, \pi^{\prime}, w\right)$.

By Section V.3.16 of [38] (see also Section 3 of [22]), the constant term of an automorphic form obtained as the iterated residue (2.6) of the Eisenstein series equals the same iterated residue of the constant term of the Eisenstein series. Therefore, by Section I.3.4 of [38], the constant term map induces an isomorphism of the space of the automorphic forms obtained as the iterated residue (2.6) and the corresponding residue of the constant term. More precisely, the following diagram is commutative:

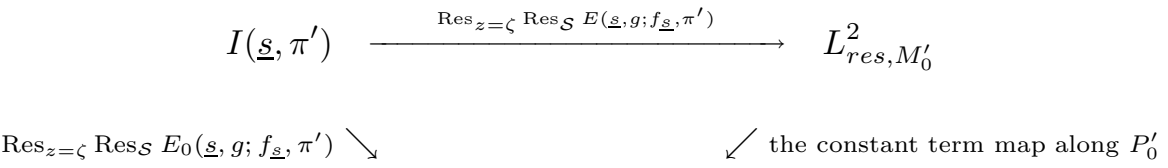


Finally, when decomposing the part of the residual spectrum $L_{\text {res, } M_{0}^{\prime}}^{2}$ attached to the cuspidal automorphic representations $\pi^{\prime}$ of $M_{0}^{\prime}(\mathbb{A})$, we consider separately the three cases A, B and C for $\pi^{\prime}$. Hence,

$$
L_{r e s, M_{0}^{\prime}}^{2} \cong L_{A}^{2} \oplus L_{B}^{2} \oplus L_{C}^{2},
$$

where $L_{X}^{2}$, for $X=A, B, C$, is the Hilbert space direct sum of the residual parts $L_{r e s,\left(M_{0}^{\prime}, \pi^{\prime}\right)}^{2}$ of the spaces $L_{\left(M_{0}^{\prime}, \pi^{\prime}\right)}^{2}$ where $\pi^{\prime}$ is a case X cuspidal automorphic representation of $M_{0}^{\prime}(\mathbb{A})$.

Before proceeding to the calculation we collect a few facts repeatedly used in the sequel. The first is just an elementary fact and we omit the proof here.

LEMMA 2.8. Let $L(s)$ be a meromorphic function on $\mathbb{C}$ having only simple poles, $L(0) \neq 0$, and satisfying the functional equation $L(s)=\varepsilon(s) L(1-s)$, where $\varepsilon(s)$ is an entire non-vanishing function such that $\varepsilon(0) \varepsilon(1)=1$. Then

$$
\left.\frac{L(s)}{L(1+s) \varepsilon(s)}\right|_{s=0}=\left\{\begin{aligned}
-1, & \text { if } s=0 \text { is a simple pole of } L(s), \\
1, & \text { otherwise. }
\end{aligned}\right.
$$

The following Lemma concerning the images of the normalized intertwining operators is just a simple consequence of the Langlands classification. It is a slight generalization of the observation on page 135 of [22] and will be useful in proving the irreducibility of the spaces of automorphic forms appearing in the decomposition of the residual spectrum.

LEMMA 2.9. Let $G$ be a reductive group defined over $k, \pi_{v}$ an irreducible representation of $M\left(k_{v}\right)$, where $M$ is the Levi factor of a standard parabolic subgroup $P, \underline{s} \in \mathfrak{a}_{M, \mathbb{C}}^{*}$ and $w \in W(M)$. Let $\pi_{v}$ be the unique irreducible subrepresentation of the induced representation $I_{L}^{M}\left(\underline{s}^{\prime}, \tau_{v}\right)$, where $L \subseteq M$ is the Levi factor of a standard parabolic subgroup, $\tau_{v}$ a tempered representation of $L\left(k_{v}\right)$ and $\underline{s}^{\prime} \in \mathfrak{a}_{L, \mathbb{C}}^{*}$. If there are Weyl group elements $w^{\prime}, w^{\prime \prime} \in W(L)$ such that

1. $\operatorname{Re}\left(w^{\prime-1}\left(\underline{s}+\underline{s}^{\prime}\right)\right)$ is in the positive Weyl chamber of $\mathfrak{a}_{w^{\prime-1}(L), \mathbb{C}}^{*}$, where $\underline{s}$ is identified with an element of $\mathfrak{a}_{L, \mathbb{C}}^{*}$,

2. $w^{\prime \prime} w w^{\prime}$ is the longest element of the Weyl group of $\left(G, w^{\prime-1}(L)\right)$,

3. $N\left(w\left(\underline{s}+\underline{s}^{\prime}\right), w\left(\tau_{v}\right), w^{\prime \prime}\right)$ is injective,

4. the image of $N\left(w^{\prime-1}\left(\underline{s}+\underline{s}^{\prime}\right), w^{\prime-1}\left(\tau_{v}\right), w^{\prime}\right)$ is $I\left(\underline{s}, \pi_{v}\right)$,

then the image of the normalized intertwining operator $N\left(\underline{s}, \pi_{v}, w\right)$ is irreducible.

In the special case of $G=G_{2}^{\prime}$ and the Levi factor $w^{\prime-1}(L)=M_{0}^{\prime}$ at a nonsplit place the requirement that $\operatorname{Re}\left(w^{\prime-1}\left(\underline{s}+\underline{s}^{\prime}\right)\right)=\left(s_{1}, s_{2}\right)$ is in the positive Weyl chamber can be replaced by a weaker condition $s_{1} \geqslant s_{2}>0$. In the split case of $G=\mathrm{SO}_{8}, w^{\prime-1}(L)=\mathrm{GL}_{n_{1}} \times \ldots \times \mathrm{GL}_{n_{k}}$, where $n_{1}+\ldots+n_{k}=4$, if $\tau_{v}$ is square-integrable then the requirement that $\operatorname{Re}\left(w^{\prime-1}\left(\underline{s}+\underline{s}^{\prime}\right)\right)=\left(s_{1}, \ldots, s_{k}\right)$ 
is in the positive Weyl chamber can be replaced by a weaker condition

$$
\begin{array}{r}
s_{1} \geqslant \ldots \geqslant s_{k}>0, \quad \text { if } n_{k}>1, \\
s_{1} \geqslant \ldots \geqslant s_{k-1} \geqslant\left|s_{k}\right|>0, \quad \text { if } n_{k}=1 \text { and } s_{k} \neq 0, \\
s_{1} \geqslant \ldots \geqslant s_{k-1}>\left|s_{k}\right|=0, \quad \text { if } n_{k}=1 \text { and } s_{k}=0 .
\end{array}
$$

Proof. The normalized intertwining operator

$$
N\left(w^{\prime-1}\left(\underline{s}+\underline{s}^{\prime}\right), w^{\prime-1}\left(\tau_{v}\right), w^{\prime \prime} w w^{\prime}\right)
$$

decomposes into

$$
N\left(w\left(\underline{s}+\underline{s}^{\prime}\right), w\left(\tau_{v}\right), w^{\prime \prime}\right) N\left(\underline{s}+\underline{s}^{\prime}, \tau_{v}, w\right) N\left(w^{\prime-1}\left(\underline{s}+\underline{s}^{\prime}\right), w^{\prime-1}\left(\tau_{v}\right), w^{\prime}\right) .
$$

Since the image of $N\left(w^{\prime-1}\left(\underline{s}+\underline{s}^{\prime}\right), w^{-1}\left(\tau_{v}\right), w^{\prime}\right)$ is precisely $I\left(\underline{s}, \pi_{v}\right)$ and $N\left(w\left(\underline{s}+\underline{s}^{\prime}\right), w\left(\tau_{v}\right), w^{\prime \prime}\right)$ is injective, the image of

$$
N\left(\underline{s}, \pi_{v}, w\right)=\left.N\left(\underline{s}+\underline{s}^{\prime}, \tau_{v}, w\right)\right|_{I\left(\underline{s}, \pi_{v}\right)}
$$

is isomorphic to the image of $N\left(w^{\prime-1}\left(\underline{s}+\underline{s}^{\prime}\right), w^{\prime-1}\left(\tau_{v}\right), w^{\prime \prime} w w^{\prime}\right)$. However, the image of that operator is irreducible because it is the long intertwining operator of the Langlands classification. In the special cases the weaker condition on $w^{\prime-1}\left(\underline{s}+\underline{s}^{\prime}\right)$ is exactly the condition of the Langlands classification using the fact that for $\mathrm{GL}_{n}^{\prime}\left(k_{v}\right)$ and $\mathrm{GL}_{n}\left(k_{v}\right)$ a tempered representation is fully induced from a square-integrable representation of a smaller Levi subgroup.

REMARK 2.11. In the following calculation we use the analytic properties of the various global $\mathrm{L}$-functions attached to cuspidal automorphic representations. The global Rankin-Selberg L-function $L\left(s, \pi_{1} \times \pi_{2}\right)$ of the cuspidal automorphic representations $\pi_{1}$ and $\pi_{2}$ of $\mathrm{GL}_{2}(\mathbb{A})$ has simple poles at $s=0$ and $s=1$ if $\pi_{1} \cong \widetilde{\pi}_{2}$ and it is entire otherwise. The global principal L-function $L(s, \pi)$ of a cuspidal automorphic representation $\pi$ of $\mathrm{GL}_{2}(\mathbb{A})$ is entire. The global Hecke $\mathrm{L}$-function $L(s, \mu)$ of a unitary character $\mu$ of $\mathbb{A}^{\times} / k^{\times}$has simple poles at $s=0$ and $s=1$ if $\mu$ is trivial and it is entire otherwise. All these global L-functions are non-vanishing for $\operatorname{Re}(s) \geqslant 1$. The local L-function $L\left(s, \mu_{v}\right)$ of a unitary character $\mu_{v}$ of a p-adic field $k_{v}^{\times}$has simple poles at $s=2 k \pi \sqrt{-1} / \log q, k \in \mathbb{Z}$, if $\mu_{v}$ is trivial and it is entire otherwise. It has no zeroes. Observe that the global Hecke $\mathrm{L}$-function $L(s, \mathbf{1})$ of the trivial character 1 of $\mathbb{A}^{\times} / k^{\times}$is nothing else than the Dedekind $\zeta$-function of the number field $k$.

2.2. Case $A$. In this case, both $\pi_{1}$ and $\pi_{2}$ are cuspidal automorphic representations of $\mathrm{GL}_{2}(\mathbb{A})$. In Table 2 the normalizing factors for the intertwining operators $A\left(\underline{s}, \pi^{\prime}, w\right)$ appearing in the constant term of the Eisenstein series attached to $\pi^{\prime}$ are given. In this case the local lift at non-split places is consistent with the global lift and hence by (1.2) and (1.16) in the global normalizing factors only the global $\mathrm{L}$-functions and $\varepsilon$-factors appear. Therefore, 
using Remark 2.11, the possible singular hyperplanes of the Eisenstein series are as shown in Figure 1.

\begin{tabular}{|c|c|}
\hline$w$ & $r\left(\underline{s}, \pi^{\prime}, w\right)$ \\
\hline 1 & 1 \\
\hline$w_{1}$ & $\begin{array}{c}L\left(1+s_{1}-s_{2}, \pi_{1} \times \tilde{\pi}_{2}\right) \\
\left.\pi_{1} \times \widetilde{\pi}_{2}\right) \varepsilon\left(s_{1}-s_{2}, \pi_{1} \times \widetilde{\pi}_{2}\right)\end{array}$ \\
\hline$w_{2}$ & $\frac{L\left(2 s_{2}, \omega_{\pi_{2}}\right)}{L\left(1+2 s_{2}, \omega_{\pi_{2}}\right) \varepsilon\left(2 s_{2}, \omega_{\pi_{2}}\right)}$ \\
\hline$w_{1} w_{2}$ & 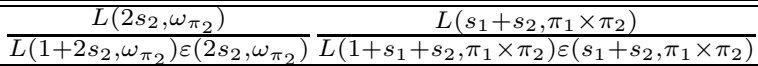 \\
\hline$w_{2} w_{1}$ &  \\
\hline$w_{1} w_{2} w_{1}$ & $\begin{array}{c}\frac{L\left(s_{1}-s_{2}, \pi_{1} \times \tilde{\pi}_{2}\right)}{L\left(1+s_{1}-s_{2}, \pi_{1} \times \widetilde{\pi}_{2}\right) \varepsilon\left(s_{1}-s_{2}, \pi_{1} \times \tilde{\pi}_{2}\right)} \\
\frac{L\left(2 s_{1}, \omega_{\pi_{1}}\right)}{L\left(1+2 s_{1}, \omega_{\pi_{1}}\right) \varepsilon\left(2 s_{1}, \omega_{\pi_{1}}\right)} \frac{L\left(s_{1}+s_{2}, \pi_{1} \times \pi_{2}\right)}{L\left(1+s_{1}+s_{2}, \pi_{1} \times \pi_{2}\right) \varepsilon\left(s_{1}+s_{2}, \pi_{1} \times \pi_{2}\right)}\end{array}$ \\
\hline$w_{2} w_{1} w_{2}$ & $\begin{array}{c}\frac{L\left(2 s_{2}, \omega_{\pi_{2}}\right)}{L\left(1+2 s_{2}, \omega_{\pi_{2}}\right) \varepsilon\left(2 s_{2}, \omega_{\pi_{2}}\right)} \\
\frac{L\left(s_{1}+s_{2}, \pi_{1} \times \pi_{2}\right)}{L\left(1+s_{1}+s_{2}, \pi_{1} \times \pi_{2}\right) \varepsilon\left(s_{1}+s_{2}, \pi_{1} \times \pi_{2}\right)} \frac{L\left(\omega_{\pi_{1}}\right)}{L\left(1+2 s_{1}, \omega_{\pi_{1}}\right) \varepsilon\left(2 s_{1}, \omega_{\pi_{1}}\right)}\end{array}$ \\
\hline$w_{1} w_{2} w_{1} w_{2}$ & 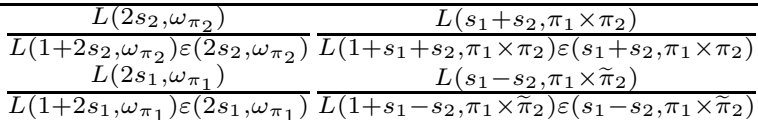 \\
\hline
\end{tabular}

TABle 2. Case A normalizing factors of $A\left(\underline{s}, \pi^{\prime}, w\right)$ for $w \in W\left(M_{0}^{\prime}\right)$

While deforming the line of integration in (2.1) from $\underline{s}_{0}$ to the origin of $\mathfrak{a}_{M_{0}^{\prime}, \mathbb{C}}^{*}$ inside the positive Weyl chamber as in Figure 1, we cross the singular hyperplanes. Taking the coordinate systems on those hyperplanes such that the origin is the orthogonal projection of the origin in $\mathfrak{a}_{M_{0}^{\prime}, \mathbb{C}}^{*}$ gives the points where the possible poles of (2.2) occur. There are three such points $A_{1}(3 / 2,1 / 2), A_{2}(1,0)$ and $A_{3}(1 / 2,1 / 2)$ in Figure 1. Points $A_{1}$ and $A_{2}$ lie on the singular hyperplane $s_{1}-s_{2}=1$ and $A_{3}$ lies on $2 s_{2}=1$.

The coordinate system on $s_{1}-s_{2}=1$ is given by $s_{1}=z+1 / 2$ and $s_{2}=z-1 / 2$, where $z$ denotes the new coordinate. Then, point $A_{1}$ corresponds to $z=1$ and $A_{2}$ to $z=1 / 2$. For the singular hyperplane $2 s_{2}=1$ the coordinate system is given by $s_{1}=z$ and $s_{2}=1 / 2$, where $z$ denotes the new coordinate. Point $A_{3}$ corresponds to $z=1 / 2$.

According to the three possible iterated poles of the Eisenstein series the part $L_{A}^{2}$ of the residual spectrum decomposes into

$$
L_{A}^{2} \cong L_{A_{1}}^{2} \oplus L_{A_{2}}^{2} \oplus L_{A_{3}}^{2},
$$

where, using the coordinate systems fixed above, $L_{A_{1}}^{2}, L_{A_{2}}^{2}$ and $L_{A_{3}}^{2}$ are obtained, respectively, as the iterated residues

$$
\operatorname{Res}_{z=1} \operatorname{Res}_{s_{1}-s_{2}=1} E\left(\underline{s}, g ; f_{\underline{s}}, \pi^{\prime}\right),
$$




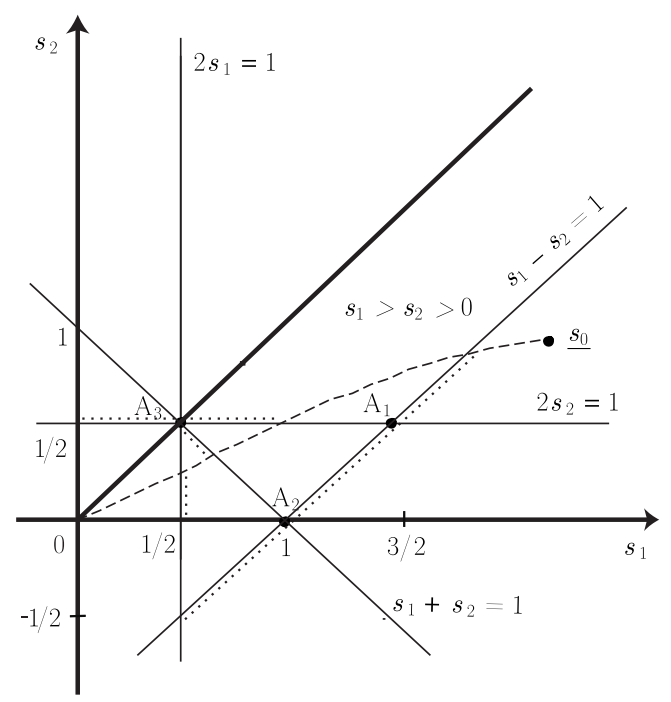

Figure 1. Case A singular hyperplanes

$$
\begin{gathered}
\operatorname{Res}_{z=1 / 2} \operatorname{Res}_{s_{1}-s_{2}=1} E\left(\underline{s}, g ; f_{\underline{s}}, \pi^{\prime}\right), \\
\operatorname{Res}_{z=1 / 2} \operatorname{Res}_{2 s_{2}=1} E\left(\underline{s}, g ; f_{\underline{s}}, \pi^{\prime}\right),
\end{gathered}
$$

of the Eisenstein series attached to the case A cuspidal automorphic representations $\pi^{\prime}$ of $M_{0}^{\prime}(\mathbb{A})$. In the following three Theorems the decomposition of those spaces is given.

THEOREM 2.12. The subspace $L_{A_{1}}^{2}$ of the residual spectrum of $G_{2}^{\prime}(\mathbb{A})$ decomposes into

$$
L_{A_{1}}^{2}=\oplus_{\pi^{\prime}} \mathcal{A}_{1}\left(\pi^{\prime}\right),
$$

where the sum is over all the cuspidal automorphic representations $\pi^{\prime} \cong \pi_{1}^{\prime} \otimes \pi_{2}^{\prime}$ of $M_{0}^{\prime}(\mathbb{A})$ such that $\pi_{1}^{\prime} \cong \pi_{2}^{\prime}$ and the central character $\omega_{\pi_{1}^{\prime}}=\omega_{\pi_{2}^{\prime}}$ is trivial.

$\mathcal{A}_{1}\left(\pi^{\prime}\right)$ is the irreducible space of automorphic forms spanned by the iterated residue

$$
\operatorname{Res}_{z=1} \operatorname{Res}_{s_{1}-s_{2}=1} E\left(\underline{s}, g ; f_{\underline{s}}, \pi^{\prime}\right)
$$

at $\underline{s}=(3 / 2,1 / 2)$ of the Eisenstein series attached to $\pi^{\prime}$. By (2.8), the constant term map induces an isomorphism of $\mathcal{A}_{1}\left(\pi^{\prime}\right)$ and the image of the normalized intertwining operator

$$
N\left((3 / 2,1 / 2), \pi^{\prime}, w_{1} w_{2} w_{1} w_{2}\right) .
$$

Proof. The contribution of the pole at point $A_{1}$ of the sum of the intertwining operators $(2.2)$ is obtained by taking the residue along $s_{1}-s_{2}=1$ first. Since, the normalized intertwining operators are holomorphic and nonvanishing in the closure of the positive Weyl chamber, the pole is obtained 
from the normalizing factors of Table 2 . The pole along the singular hyperplane $s_{1}-s_{2}=1$ occurs if and only if $\pi_{1} \cong \pi_{2}$ for the intertwining operators corresponding to the Weyl group elements $w_{1}, w_{2} w_{1}, w_{1} w_{2} w_{1}$ and $w_{1} w_{2} w_{1} w_{2}$. It is a simple pole. Up to the nonzero constant

$$
\operatorname{Res}_{s=1} L\left(s, \pi_{1} \times \widetilde{\pi}_{2}\right) L\left(2, \pi_{1} \times \tilde{\pi}_{2}\right)^{-1} \varepsilon\left(1, \pi_{1} \times \tilde{\pi}_{2}\right)^{-1},
$$

the residues along $s_{1}-s_{2}=1$ written in the new variable $z$ defined above are given in Table 3.

\begin{tabular}{|c|c|}
\hline$w$ & $\operatorname{Res}_{s_{1}-s_{2}=1} r\left(\underline{s}, \pi^{\prime}, w\right)$ \\
\hline \hline$w_{1}$ & 1 \\
\hline \hline$w_{2} w_{1}$ & $\frac{L\left(1+2 z, \omega_{\pi_{1}}\right)}{L\left(2+2 z, \omega_{\pi_{1}}\right) \varepsilon\left(1+2 z, \omega_{\pi_{1}}\right)}$ \\
\hline \hline$w_{1} w_{2} w_{1}$ & $\frac{L\left(2 z, \pi_{1} \times \pi_{2}\right)}{L\left(2+2 z, \omega_{\pi_{1}}\right) \varepsilon\left(1+2 z, \omega_{\pi_{1}}\right)} \frac{L\left(1+2 z, \pi_{1} \times \pi_{2}\right) \varepsilon\left(2 z, \pi_{1} \times \pi_{2}\right)}{L\left(1+1+2 z, \omega_{\pi_{2}}\right)}$ \\
\hline \hline$w_{1} w_{2} w_{1} w_{2}$ & $\frac{L\left(2 z, \omega_{\pi_{2}}\right) \varepsilon\left(-1+2 z, \omega_{\pi_{2}}\right)}{L\left(1+2 z, \omega_{\pi_{1}}\right)}$ \\
& $\frac{L\left(2 z, \pi_{1} \times \pi_{2}\right)}{L\left(1+2 z, \pi_{1} \times \pi_{2}\right) \varepsilon\left(2 z, \pi_{1} \times \pi_{2}\right)} \frac{L\left(2+2 z, \omega_{\pi_{1}}\right) \varepsilon\left(1+2 z, \omega_{\pi_{1}}\right)}{L(2+2)}$ \\
\hline
\end{tabular}

TABLE 3. Residues along $s_{1}-s_{2}=1$ of case A normalizing factors

For the pole at point $A_{1}$ we must look at the pole of the terms in Table 3 at $z=1$. The pole at $z=1$ occurs if and only if $\omega_{\pi_{2}}=1$ for the term corresponding to $w_{1} w_{2} w_{1} w_{2}$ and it is simple. Hence, the pole of the Eisenstein series at point $A_{1}$ occurs if and only if $\pi_{1} \cong \pi_{2}$ and $\omega_{\pi_{1}}=\omega_{\pi_{2}}$ is trivial, i.e. if and only if $\pi_{1}^{\prime} \cong \pi_{2}^{\prime}$ and $\omega_{\pi_{1}^{\prime}}=\omega_{\pi_{2}^{\prime}}$ is trivial. In that case the residue of the constant term is up to a nonzero constant equal to the normalized intertwining operator $N\left((3 / 2,1 / 2), \pi^{\prime}, w_{1} w_{2} w_{1} w_{2}\right)$. The square integrability follows from Lemma 2.7 because $w_{1} w_{2} w_{1} w_{2}(3 / 2,1 / 2)=(-3 / 2,-1 / 2)$.

It remains to prove that the image of that operator is irreducible. Using Lemma 2.9 we prove the irreducibility of the image of the local operators $N\left((3 / 2,1 / 2), \pi_{v}^{\prime}, w_{1} w_{2} w_{1} w_{2}\right)$ at every place $v$. Let $w=w_{1} w_{2} w_{1} w_{2}$ and $\underline{s}=$ $(3 / 2,1 / 2)$. At all the places where the representation $\pi_{v}^{\prime}$ is tempered the image is irreducible by the Langlands classification since $\underline{s}$ is in the positive Weyl chamber and $w$ is the longest Weyl group element for $\left(G_{2}^{\prime}, M_{0}^{\prime}\right)$. Observe that this is the case for all non-split places.

For a split place where the representation $\pi_{v} \cong \pi_{1, v} \otimes \pi_{2, v}$ is non-tempered at least one of representations $\pi_{i, v}$ is a complementary series, i.e.

$$
\pi_{i, v} \cong \operatorname{Ind}_{\mathrm{GL}_{1}\left(k_{v}\right) \times \mathrm{GL}_{1}\left(k_{v}\right)}^{\mathrm{GL}_{2}\left(k_{v}\right)}\left(\mu_{i, v}|\cdot|^{r_{i}} \otimes \mu_{i, v}|\cdot|^{-r_{i}}\right)
$$

where $0<r_{i}<1 / 2$ and $\mu_{i, v}$ is a unitary character of $k_{v}^{\times}$. Then $\pi_{v}$ is a fully induced representation from a tempered representation of a smaller parabolic 
subgroup. In the notation of Lemma 2.9 the Levi factor $L$ is one of the Levi factors $\mathrm{GL}_{1} \times \mathrm{GL}_{1} \times \mathrm{GL}_{2}, \mathrm{GL}_{2} \times \mathrm{GL}_{1} \times \mathrm{GL}_{1}, \mathrm{GL}_{1} \times \mathrm{GL}_{1} \times \mathrm{GL}_{1} \times \mathrm{GL}_{1}$ and

$$
\underline{s}+\underline{s}^{\prime}=\left\{\begin{array}{l}
\left(3 / 2+r_{1}, 3 / 2-r_{1}, 1 / 2\right), \\
\left(3 / 2,1 / 2+r_{2}, 1 / 2-r_{2}\right), \\
\left(3 / 2+r_{1}, 3 / 2-r_{1}, 1 / 2+r_{2}, 1 / 2-r_{2}\right),
\end{array}\right.
$$

where $0<r_{i}<1 / 2$. Let $w^{\prime}=1$ and in notation of the proof of Proposition 1.12

$$
w^{\prime \prime}=\left\{\begin{array}{l}
(1,2)(3), \\
(1)(2,3), \\
(1,2)(3,4) .
\end{array}\right.
$$

Then $w^{\prime \prime} w w^{\prime}$ is the longest Weyl group element for $\left(\mathrm{SO}_{8}, L\right), \underline{s}+\underline{s}^{\prime}$ is in the positive Weyl chamber and $N\left(w(\underline{s}), w\left(\pi_{v}\right), w^{\prime \prime}\right)=N\left(w\left(\underline{s}^{\prime} \underline{s}^{\prime}\right), w\left(\tau_{v}\right), w^{\prime \prime}\right)$ is an isomorphism. Hence, by Lemma 2.9, the image of the normalized intertwining operator $N\left((3 / 2,1 / 2), \pi_{v}, w\right)$ is irreducible.

Before giving the decomposition of $L_{A_{2}}^{2}$ we consider the induced representation $\operatorname{Ind}_{\mathrm{GL}_{1}^{\prime}\left(k_{v}\right)}^{G_{1}^{\prime}\left(k_{v}\right)} \sigma_{v}^{\prime}$ of $G_{1}^{\prime}\left(k_{v}\right)$ and the corresponding normalized intertwining operator $N\left(0, \sigma_{v}^{\prime}, w_{2}\right)$, where $\sigma_{v}^{\prime}$ is a selfcontragredient irreducible unitary representation of $\mathrm{GL}_{1}^{\prime}\left(k_{v}\right)$. We exploit the structure of the group $G_{1}^{\prime}$ (see also $[17])$.

The group $G_{1}^{\prime}$ as an algebraic group over $k$ can be realized as

$$
G_{1}^{\prime}=\left\{\left[\begin{array}{cc}
a g & b g \\
c g & d g
\end{array}\right] \in \mathrm{GL}_{2}^{\prime}: g \in \mathrm{GL}_{1}^{\prime},\left[\begin{array}{cc}
a & b \\
c & d
\end{array}\right] \in \mathrm{GL}_{2},(a d-b c) \operatorname{det}_{v}^{\prime}(g)=1\right\},
$$

where $\operatorname{det}_{v}^{\prime}$ is the determinant over split places and the reduced norm over non-split places. Hence, $G_{1}^{\prime}$ has the following two subgroups

$$
\mathrm{SL}_{1}^{\prime(1)}=\left\{\left[\begin{array}{ll}
g & 0 \\
0 & g
\end{array}\right] \in \mathrm{GL}_{2}^{\prime}: g \in \mathrm{SL}_{1}^{\prime}\right\}
$$

and

$$
\mathrm{SL}_{2}^{(2)}=\left\{\left[\begin{array}{ll}
a I_{1}^{\prime} & b I_{1}^{\prime} \\
c I_{1}^{\prime} & d I_{1}^{\prime}
\end{array}\right] \in \mathrm{GL}_{2}^{\prime}:\left[\begin{array}{ll}
a & b \\
c & d
\end{array}\right] \in \mathrm{SL}_{2}\right\},
$$

where $I_{n}^{\prime}$ equals the $2 n \times 2 n$ identity matrix at split places and $I_{n}^{\prime}$ equals the $n \times n$ identity matrix at non-split places. The intersection of these subgroups is $\left\{ \pm I_{2}^{\prime}\right\}$ and the product $\mathrm{SL}_{1}^{\prime(1)} \mathrm{SL}_{2}^{(2)}$ is a normal subgroup of $G_{1}^{\prime}$. Over a local field $k_{v}$ the quotient is finite and isomorphic to $k_{v}^{\times} /\left(k_{v}^{\times}\right)^{2}$. The following Lemma considers the restriction of representations of $G_{1}^{\prime}\left(k_{v}\right)$ to $\mathrm{SL}_{1}^{\prime(1)}\left(k_{v}\right) \mathrm{SL}_{2}^{(2)}\left(k_{v}\right)$.

Let $\left\{\gamma_{v}\right\}$ be the fixed set of representatives of $k_{v}^{\times} /\left(k_{v}^{\times}\right)^{2}$. Abusing the notation, let $\gamma_{v}^{\prime}$ at split places denote in the same time diagonal matrices

$$
\operatorname{diag}\left(\gamma_{v}, 1\right) \in \mathrm{GL}_{2}\left(k_{v}\right) \text { and } \operatorname{diag}\left(\gamma_{v}, 1,1, \gamma_{v}^{-1}\right) \in \mathrm{SO}_{4}\left(k_{v}\right),
$$


while at non-split places it denotes in the same time the element of $\mathrm{GL}_{1}^{\prime}\left(k_{v}\right) \cong$ $D_{v}^{\times}$corresponding to $\operatorname{diag}\left(\gamma_{v}, 1\right) \in \mathrm{GL}_{2}\left(k_{v}\right)$ in a matrix representation of $D_{v}$ as in Section 2 of [16] and the matrix

$$
\operatorname{diag}\left(\gamma_{v}^{\prime}, \gamma_{v}^{-1} \gamma_{v}^{\prime}\right) \in G_{1}^{\prime}\left(k_{v}\right) .
$$

This notation is justified by the fact that the conjugation in the group $\mathrm{SL}_{1}^{\prime(1)}\left(k_{v}\right) \mathrm{SL}_{2}^{(2)}\left(k_{v}\right)$ by $\gamma_{v}^{\prime}$ as an element of $G_{1}^{\prime}\left(k_{v}\right)$ is actually the conjugation by $\gamma_{v}^{\prime}$ as an element of $\mathrm{GL}_{1}^{\prime}\left(k_{v}\right)$ in $\mathrm{SL}_{1}^{\prime(1)}\left(k_{v}\right)$ and $\mathrm{SL}_{2}^{(2)}\left(k_{v}\right)$. Observe that $\left\{\gamma_{v}^{\prime}\right\}$ forms in the same time the set of representatives for

$$
G_{1}^{\prime}\left(k_{v}\right) / \mathrm{SL}_{1}^{\prime(1)}\left(k_{v}\right) \mathrm{SL}_{2}^{(2)}\left(k_{v}\right) \text { and } \mathrm{GL}_{1}^{\prime}\left(k_{v}\right) / \mathrm{SL}_{1}^{\prime}\left(k_{v}\right) Z\left(k_{v}\right),
$$

where $Z$ is the center of $\mathrm{GL}_{1}^{\prime}$. These facts concerning the conjugation reduce the proof of the following Lemma to Lemmas 2.4, 2.5 and 2.8 of [29]. See also [49].

LEMma 2.14. Let $\pi_{v}^{\prime}$ be an irreducible representation of $G_{1}^{\prime}\left(k_{v}\right)$. The restriction of $\pi_{v}^{\prime}$ to the subgroup $\mathrm{SL}_{1}^{\prime(1)}\left(k_{v}\right) \mathrm{SL}_{2}^{(2)}\left(k_{v}\right)$ decomposes into

$$
\left.\pi_{v}^{\prime}\right|_{\mathrm{SL}_{1}^{\prime(1)}\left(k_{v}\right) \mathrm{SL}_{2}^{(2)}\left(k_{v}\right)} \cong \bigoplus_{\gamma_{v}^{\prime} \in \Gamma_{v}^{\prime}}\left(\sigma_{1, v}^{\prime \gamma_{v}^{\prime}} \otimes \sigma_{2, v}^{\gamma_{v}^{\prime}}\right)
$$

where $\sigma_{1, v}^{\prime} \otimes \sigma_{2, v}$ is any irreducible subrepresentation of the above restriction, $\sigma_{i, v}^{\gamma_{v}^{\prime}}$ denotes representation $\sigma_{i, v}^{\gamma_{v}^{\prime}}(g)=\sigma_{i, v}\left(\gamma_{v}^{\prime-1} g \gamma_{v}^{\prime}\right)$ and the sum is over the subset $\Gamma_{v}^{\prime}$ of the set of all representatives $\gamma_{v}^{\prime}$ such that different $\sigma_{1, v}^{\prime \gamma_{v}^{\prime}} \otimes \sigma_{2, v}^{\gamma_{v}^{\prime}}$ are not isomorphic.

Conversely, for an irreducible representation $\sigma_{1, v}^{\prime} \otimes \sigma_{2, v}$ of the subgroup $\mathrm{SL}_{1}^{\prime(1)}\left(k_{v}\right) \mathrm{SL}_{2}^{(2)}\left(k_{v}\right)$ there is an irreducible representation $\pi_{v}^{\prime}$ of $G_{1}^{\prime}\left(k_{v}\right)$ such that

$$
\left.\sigma_{1, v}^{\prime} \otimes \sigma_{2, v} \quad \subset \quad \pi_{v}^{\prime}\right|_{\mathrm{SL}_{1}^{\prime(1)}\left(k_{v}\right) \mathrm{SL}_{2}^{(2)}\left(k_{v}\right)} .
$$

It is unique up to a quadratic character of $G_{1}^{\prime}\left(k_{v}\right)$ which is trivial on $\mathrm{SL}_{1}^{\prime(1)}\left(k_{v}\right) \mathrm{SL}_{2}^{(2)}\left(k_{v}\right)$.

LEMMA 2.15. Let $\pi_{v}^{\prime}=\operatorname{Ind}_{\mathrm{GL}_{1}^{\prime}\left(k_{v}\right)}^{G_{1}^{\prime}\left(k_{v}\right)} \sigma_{v}^{\prime}$, where $\sigma_{v}^{\prime}$ is a selfcontragredient irreducible unitary representation of $\mathrm{GL}_{1}^{\prime}\left(k_{v}\right)$. Then in the notation of Lemma 2.14 the restriction of $\pi_{v}^{\prime}$ to the subgroup $\mathrm{SL}_{1}^{\prime(1)}\left(k_{v}\right) \mathrm{SL}_{2}^{(2)}\left(k_{v}\right)$ decomposes into

$$
\left.\pi_{v}^{\prime}\right|_{\mathrm{SL}_{1}^{\prime(1)}\left(k_{v}\right) \mathrm{SL}_{2}^{(2)}\left(k_{v}\right)} \cong\left(\oplus_{\gamma_{v}^{\prime} \in \Gamma_{v}^{\prime}} \tau_{v}^{\prime \gamma_{v}^{\prime}}\right) \otimes \operatorname{Ind}_{\mathrm{GL}_{1}\left(k_{v}\right)}^{\mathrm{SL}_{2}^{(2)}\left(k_{v}\right)} \omega_{\sigma_{v}^{\prime}}
$$

where $\tau_{v}^{\prime}$ is a subrepresentation of the restriction of $\sigma_{v}^{\prime}$ from $\mathrm{GL}_{1}^{\prime}\left(k_{v}\right)$ to $\mathrm{SL}_{1}^{\prime(1)}\left(k_{v}\right)$ and $\omega_{\sigma_{v}^{\prime}}$ is the central character of $\sigma_{v}^{\prime}$. Here $\Gamma_{v}^{\prime}$ is the set of the representatives $\gamma_{v}^{\prime}$ such that different $\tau_{v}^{\gamma_{v}^{\prime}}$ are not isomorphic. Therefore, $\pi_{v}^{\prime}$ 
is irreducible if $\omega_{\sigma_{v}^{\prime}}$ is trivial and it is the direct sum of two non-isomorphic irreducible components otherwise.

Proof. The application of the Mackey theory (similar to Corollary 5.3.4.2 of [52]) to our finite index subgroup case shows that

$$
\left.\left(\operatorname{Ind}_{\mathrm{GL}_{1}^{\prime}\left(k_{v}\right)}^{G^{\prime}\left(k_{v}\right)} \sigma_{v}^{\prime}\right)\right|_{\mathrm{SL}_{1}^{\prime(1)}\left(k_{v}\right) \mathrm{SL}_{2}^{(2)}\left(k_{v}\right)} \cong \operatorname{Ind}_{\mathrm{SL}_{1}^{\prime(1)}\left(k_{v}\right) T^{(2)}\left(k_{v}\right)}^{\mathrm{SL}_{(1)}^{\prime(1)}\left(k_{v}\right) \mathrm{SL}_{(2)}^{(2)}\left(k_{v}\right)}\left(\left.\sigma_{v}^{\prime}\right|_{\mathrm{SL}_{1}^{\prime(1)}\left(k_{v}\right) T^{(2)}\left(k_{v}\right)}\right),
$$

where $T^{(2)}$ is the maximal split torus in $\mathrm{SL}_{2}^{(2)}$ and $\mathrm{SL}_{1}^{\prime(1)} T^{(2)}$ is isomorphic to $\mathrm{GL}_{1}^{\prime} \cap \mathrm{SL}_{1}^{\prime(1)} \mathrm{SL}_{2}^{(2)}$. Since in our matrix realization $\mathrm{SL}_{1}^{\prime(1)}$ is just the $\mathrm{SL}_{1}^{\prime}$ inside the Levi factor $\mathrm{GL}_{1}^{\prime}$ and $T^{(2)}$ is the center of $\mathrm{GL}_{1}^{\prime}$, the restriction on the right hand side decomposes into the finite direct sum

$$
\left(\oplus_{\gamma_{v}^{\prime} \in \Gamma_{v}^{\prime}} \tau_{v}^{\prime \gamma_{v}^{\prime}}\right) \otimes \omega_{\sigma_{v}^{\prime}}
$$

where $\tau_{v}^{\prime}$ is an irreducible subrepresentation of the restriction of $\sigma_{v}^{\prime}$ to $\mathrm{SL}_{1}^{\prime(1)}$ (see Lemma 2.14) and $\omega_{\sigma_{v}^{\prime}}$ is the central character of $\sigma_{v}^{\prime}$. Hence, we obtain

$$
\left.\pi_{v}^{\prime}\right|_{\mathrm{SL}_{1}^{\prime(1)}\left(k_{v}\right) \mathrm{SL}_{2}^{(2)}\left(k_{v}\right)} \cong\left(\oplus_{\gamma_{v}^{\prime} \in \Gamma_{v}^{\prime}} \tau_{v}^{\prime \gamma_{v}^{\prime}}\right) \otimes \operatorname{Ind}_{T^{(2)}\left(k_{v}\right)}^{\mathrm{SL}^{(2)}\left(k_{v}\right)} \omega_{\sigma_{v}^{\prime}}
$$

as claimed. Now, if $\omega_{\sigma_{v}^{\prime}}$ is trivial the induced representation $\operatorname{Ind}_{T^{(2)}\left(k_{v}\right)}^{\mathrm{SL}_{2}^{(2)}\left(k_{v}\right)} \omega_{\sigma_{v}^{\prime}}$ is irreducible and the restriction of $\pi_{v}^{\prime}$ becomes

$$
\oplus_{\gamma_{v}^{\prime} \in \Gamma_{v}^{\prime}}\left(\tau_{v}^{\prime \gamma_{v}^{\prime}} \otimes \operatorname{Ind}_{T^{(2)}\left(k_{v}\right)}^{\mathrm{SL}^{(2)}\left(k_{v}\right)} \omega_{\sigma_{v}^{\prime}}\right)
$$

which is the restriction of an irreducible representation of $G_{1}^{\prime}\left(k_{v}\right)$. If $\omega_{\sigma_{v}^{\prime}}$ is not trivial the induced representation $\operatorname{Ind}_{T_{2}^{(2)}\left(k_{v}\right)}^{\mathrm{SL}^{(2)}\left(k_{v}\right)} \omega_{\sigma_{v}^{\prime}}$ is the direct sum of two irreducible representations of $\mathrm{SL}_{2}^{(2)}$ which are conjugate by a representative $\gamma_{v}^{\prime}$. Denote those representations by $\tau^{+}$and $\tau^{-}$. The restriction of $\pi_{v}^{\prime}$ can be written as

$$
\left[\oplus_{\gamma_{v}^{\prime} \in \Gamma_{v}^{\prime}}\left(\tau_{v}^{\prime \gamma_{v}^{\prime}} \otimes\left(\tau^{+}\right)^{\gamma_{v}^{\prime}}\right)\right] \oplus\left[\oplus_{\gamma_{v}^{\prime} \in \Gamma_{v}^{\prime}}\left(\tau_{v}^{\prime \gamma_{v}^{\prime}} \otimes\left(\tau^{-}\right)^{\gamma_{v}^{\prime}}\right)\right] .
$$

where every square bracket is the restriction of an irreducible representation of $G_{1}^{\prime}\left(k_{v}\right)$. Hence, in this case $\pi_{v}^{\prime}$ is the direct sum of two non-isomorphic irreducible representations.

COROLLARY 2.16. In the notation as above, the eigenspaces of the normalized intertwining operator $N\left(0, \sigma_{v}^{\prime}, w_{2}\right)$ acting on the induced representation $\pi_{v}^{\prime} \cong \operatorname{Ind}_{\mathrm{GL}_{1}^{\prime}\left(k_{v}\right)}^{G_{1}^{\prime}\left(k_{v}\right)} \sigma_{v}^{\prime}$ are irreducible. More precisely,

$$
\pi_{v}^{\prime} \cong \pi_{v}^{\prime+} \oplus \pi_{v}^{\prime-}
$$


where, $\pi_{v}^{\prime \pm}$ denotes the irreducible eigenspace corresponding to the eigenvalue \pm 1 , and one of $\pi_{v}^{\prime \pm}$ is trivial if and only if $\omega_{\sigma_{v}^{\prime}}$ is trivial. If $v$ is a split place and $\sigma_{v}^{\prime}$ is unramified, then the unramified component of $\pi_{v}^{\prime}$ is always $\pi_{v}^{\prime+}$.

Consider the induced representation

$\operatorname{Ind}_{\mathrm{GL}_{1}^{\prime}\left(k_{v}\right) \times \mathrm{GL}_{1}^{\prime}\left(k_{v}\right)}^{G_{2}^{\prime}\left(k_{v}\right)}\left(\pi_{1, v}^{\prime} \nu \otimes \pi_{2, v}^{\prime}\right) \cong \operatorname{Ind}_{\mathrm{GL}_{1}^{\prime}\left(k_{v}\right) \times G_{1}^{\prime}\left(k_{v}\right)}^{G^{\prime}\left(k_{v}\right)}\left(\pi_{1, v}^{\prime} \nu \otimes \operatorname{Ind}_{\mathrm{GL}_{1}^{\prime}\left(k_{v}\right)}^{G_{1}^{\prime}\left(k_{v}\right)} \pi_{2, v}^{\prime}\right)$.

According to Lemma 2.15 and its Corollary, it decomposes into the direct sum of irreducible representations

$$
\bigoplus_{\eta_{v} \in\{+,-\}} \operatorname{Ind}_{\mathrm{GL}_{1}^{\prime}\left(k_{v}\right) \times G_{1}^{\prime}\left(k_{v}\right)}^{G_{2}^{\prime}\left(k_{v}\right)}\left(\pi_{1, v}^{\prime} \nu \otimes\left(\operatorname{Ind}_{\mathrm{GL}_{1}^{\prime}\left(k_{v}\right)}^{G_{1}^{\prime}\left(k_{v}\right)} \pi_{2, v}^{\prime}\right)^{\eta_{v}}\right)
$$

where one of the components is trivial if and only if the central character $\omega_{\pi_{2, v}^{\prime}}$ is trivial. The image of the normalized intertwining operator $N\left((1,0), \pi_{v}^{\prime}, w_{1} w_{2} w_{1}\right)$ acting on each of the irreducible components is denoted by $\Pi_{v}^{\prime \eta_{v}}$, where $\eta_{v} \in\{+,-\}$. Observe that one of $\Pi_{v}^{\prime \eta_{v}}$ is trivial if and only if the central character $\omega_{\pi_{2, v}^{\prime}}$ is trivial.

THEOREM 2.17. The subspace $L_{A_{2}}^{2}$ of the residual spectrum of $G_{2}^{\prime}(\mathbb{A})$ decomposes into

$$
L_{A_{2}}^{2}=\left(\oplus_{\pi^{\prime}} \mathcal{A}_{2}^{(1)}\left(\pi^{\prime}\right)\right) \oplus\left(\oplus_{\pi^{\prime}} \mathcal{A}_{2}^{(2)}\left(\pi^{\prime}\right)\right) .
$$

The former sum is over all the cuspidal automorphic representations $\pi^{\prime} \cong$ $\pi_{1}^{\prime} \otimes \pi_{2}^{\prime}$ of $M_{0}^{\prime}(\mathbb{A})$ such that $\pi_{1}^{\prime} \cong \pi_{2}^{\prime}$ is selfcontragredient, the central character $\omega_{\pi_{1}^{\prime}}=\omega_{\pi_{2}^{\prime}}$ is trivial and the parity condition $\prod_{v} \eta_{v}=-1$ holds. The latter sum is over all the cuspidal automorphic representations $\pi^{\prime} \cong \pi_{1}^{\prime} \otimes \pi_{2}^{\prime}$ of $M_{0}^{\prime}(\mathbb{A})$ such that $\pi_{1}^{\prime} \cong \pi_{2}^{\prime}$ is selfcontragredient and the central character $\omega_{\pi_{1}^{\prime}}=\omega_{\pi_{2}^{\prime}}$ is nontrivial.

Both $\mathcal{A}_{2}^{(1)}\left(\pi^{\prime}\right)$ and $\mathcal{A}_{2}^{(2)}\left(\pi^{\prime}\right)$ are the spaces of automorphic forms spanned by the iterated residues

$$
\operatorname{Res}_{z=1 / 2} \operatorname{Res}_{s_{1}-s_{2}=1} E\left(\underline{s}, g ; f_{\underline{s}}, \pi^{\prime}\right)
$$

at $\underline{s}=(1,0)$ of the Eisenstein series attached to $\pi^{\prime} \cdot \mathcal{A}_{2}^{(1)}\left(\pi^{\prime}\right)$ is irreducible and, by (2.8), the constant term map induces an isomorphism of $\mathcal{A}_{2}^{(1)}\left(\pi^{\prime}\right)$ and the image of the normalized intertwining operator

$$
N\left((1,0), \pi^{\prime}, w_{1} w_{2} w_{1}\right) .
$$

By (2.8), the constant term map induces an isomorphism of $\mathcal{A}_{2}^{(2)}\left(\pi^{\prime}\right)$ and the sum of the irreducible representations of the form $\otimes_{v} \Pi_{v}^{\prime \eta_{v}}$, where $\eta_{v}$ is one of the two possible signs at places where $\omega_{\pi_{1, v}}=\omega_{\pi_{2, v}}$ is nontrivial, at almost all places $\eta_{v}=+$ and the product of all the signs $\prod_{v} \eta_{v}=1$. 
Proof. The iterated residue at $A_{2}$, as shown in Figure 1, is calculated first along $s_{1}-s_{2}=1$. The residues written in the new variable $z$ were calculated in the proof of the previous Theorem and given in Table 3 up to a nonzero constant. They appear if and only if $\pi_{1} \cong \pi_{2}$. The pole at point $A_{2}$ is actually the pole at $z=1 / 2$. From the analytic properties of the $\mathrm{L}$-functions involved it follows that the simple pole at $z=1 / 2$ occurs if and only if $\pi_{1} \cong \widetilde{\pi}_{2}$ for the intertwining operators corresponding to the Weyl group elements $w_{1} w_{2} w_{1}$ and $w_{1} w_{2} w_{1} w_{2}$. Observe that $\pi_{1} \cong \pi_{2}$ are selfcontragredient and hence their central characters are quadratic. Up to the nonzero constant

$$
\frac{\operatorname{Res}_{s=1} L\left(s, \pi_{1} \times \pi_{2}\right) L\left(2, \omega_{\pi_{1}}\right)}{L\left(2, \pi_{1} \times \pi_{2}\right) \varepsilon\left(1, \pi_{1} \times \pi_{2}\right) L\left(3, \omega_{\pi_{1}}\right) \varepsilon\left(2, \omega_{\pi_{1}}\right)},
$$

the former residue equals $N\left((1,0), \pi^{\prime}, w_{1} w_{2} w_{1}\right)$. For the latter we distinguish two cases depending on $\omega_{\pi_{2}}$.

If the quadratic central character $\omega_{\pi_{2}}$ is trivial then the Hecke L-function $L\left(s, \omega_{\pi_{2}}\right)$ has the simple pole at $s=0, s=1$ and by Lemma 2.8 the latter residue, up to the same constant above, equals $-N\left((1,0), \pi^{\prime}, w_{1} w_{2} w_{1} w_{2}\right)$. After decomposing according to Proposition 1.4 and acting on $\otimes_{v} f_{v}$, the residue at $A_{2}$ in this case, up to a nonzero constant, equals

$$
\otimes_{v} N\left((1,0), \pi_{v}^{\prime}, w_{1} w_{2} w_{1}\right)\left[\otimes_{v} f_{v}-\otimes_{v} N\left((1,0), \pi_{v}^{\prime}, w_{2}\right) f_{v}\right],
$$

where the intertwining operator $N\left((1,0), \pi_{v}^{\prime}, w_{2}\right)$ is actually the $G_{1}^{\prime}\left(k_{v}\right)$ intertwining operator for $\operatorname{Ind}_{\mathrm{GL}_{1}^{\prime}\left(k_{v}\right)}^{G_{2}^{\prime}\left(k_{v}\right)} \pi_{2, v}^{\prime}$. Since $\omega_{\pi_{2, v}}=\omega_{\pi_{2, v}^{\prime}}$ is trivial, the discussion before the statement of the Theorem implies that this intertwining operator acts as $\eta_{v} I d_{v}$, where $\eta_{v} \in\{ \pm 1\}$ is uniquely determined at every place. Therefore, the residue in this case is non-vanishing if $\prod_{v} \eta_{v}=-1$ and it is the image of the normalized intertwining operator $N\left((1,0), \pi^{\prime}, w_{1} w_{2} w_{1}\right)$. The irreducibility of that image is obtained at the end of the proof.

If the quadratic central character $\omega_{\pi_{2}}$ is nontrivial then the Hecke Lfunction $L\left(s, \omega_{\pi_{2}}\right)$ is entire and by Lemma 2.8 the latter residue equals $N\left((1,0), \pi^{\prime}, w_{1} w_{2} w_{1} w_{2}\right)$ up to the same constant above. Again, after decomposing according to Proposition 1.4 and acting on $\otimes_{v} f_{v}$, the residue at $A_{2}$, up to the nonzero constant, equals

$$
\otimes_{v} N\left((1,0), \pi_{v}^{\prime}, w_{1} w_{2} w_{1}\right)\left[\otimes_{v} f_{v}+\otimes_{v} N\left((1,0), \pi_{v}^{\prime}, w_{2}\right) f_{v}\right] .
$$

The discussion before the statement of the Theorem shows that the $G_{1}^{\prime}\left(k_{v}\right)$ intertwining operator $N\left((1,0), \pi_{v}^{\prime}, w_{2}\right)$ acts on $f_{v}$ as $\eta_{v} I d_{v}$ if

$$
f_{v} \in\left(\operatorname{Ind}_{\mathrm{GL}_{1}^{\prime}\left(k_{v}\right)}^{G_{1}^{\prime}\left(k_{v}\right)} \pi_{2, v}^{\prime}\right)^{\eta_{v}}
$$

where $\eta_{v} \in\{+,-\}$. Since $f_{v}$ is unramified at almost all places, the operator acts as the identity at those places, i.e. $\eta_{v}=+$. If the product of the 
corresponding signs $\prod_{v} \eta_{v}=-1$, then the residue vanishes. This gives the last condition of the Theorem.

Since $w_{1} w_{2} w_{1} w_{2}(1,0)=w_{1} w_{2} w_{1}(1,0)=(-1,0)$, by Lemma 2.7 , the automorphic forms obtained as the residues are square-integrable. It remains to prove that $\mathcal{A}_{2}^{(1)}\left(\pi^{\prime}\right)$ and $\otimes_{v} \Pi_{v}^{\prime \eta_{v}}$ are irreducible. It is enough to prove the irreducibility of $\Pi_{v}^{\prime \eta_{v}}$ at every place $v$. But $\Pi_{v}^{\prime \eta_{v}}$ is the image of the normalized intertwining operator $N\left((1,0), \pi_{v}^{\prime}, w_{1} w_{2} w_{1}\right)$ acting on

$$
\operatorname{Ind}_{\mathrm{GL}_{1}^{\prime}\left(k_{v}\right) \times G_{1}^{\prime}\left(k_{v}\right)}^{G_{2}^{\prime}\left(k_{v}\right)}\left(\pi_{1, v}^{\prime} \nu \otimes\left(\operatorname{Ind}_{\mathrm{GL}_{1}^{\prime}\left(k_{v}\right)}^{G_{1}^{\prime}\left(k_{v}\right)} \pi_{2, v}^{\prime}\right)^{\eta_{v}}\right) .
$$

If $\pi_{1, v}^{\prime}$ and $\pi_{2, v}^{\prime}$ are tempered then $\left(\operatorname{Ind}_{\mathrm{GL}_{1}^{\prime}\left(k_{v}\right)}^{G_{1}^{\prime}\left(k_{v}\right)} \pi_{2, v}^{\prime}\right)^{\eta_{v}}$ is also tempered and the image is irreducible by the Langlands classification since $w_{1} w_{2} w_{1}$ is the longest Weyl group element for $\left(G_{2}^{\prime}, \mathrm{GL}_{1}^{\prime} \times G_{1}^{\prime}\right)$ and 1 is in the positive Weyl chamber. Observe that this is the case at all non-split places.

Otherwise, at least one of the representations $\pi_{i, v}$ of $\mathrm{GL}_{2}\left(k_{v}\right)$ is a complementary series

$$
\pi_{i, v} \cong \operatorname{Ind}_{\mathrm{GL}_{1}\left(k_{v}\right) \times \mathrm{GL}_{1}\left(k_{v}\right)}^{\mathrm{GL}_{2}\left(k_{v}\right)}\left(\mu_{i, v}|\cdot|^{r_{i}} \otimes \mu_{i, v}|\cdot|^{-r_{i}}\right),
$$

where $0<r_{i}<1 / 2$ and $\mu_{i, v}$ are unitary quadratic characters since $\pi_{i, v}$ are selfcontragredient. But then, by Lemma 2.15, the induced representation $\operatorname{Ind}_{\mathrm{GL}_{2}\left(k_{v}\right)}^{\mathrm{SO}_{4}\left(k_{v}\right)}\left(\pi_{2, v}\right)$ is irreducible since $\omega_{\pi_{2, v}}$ is trivial. Hence, the unique nontrivial eigenspace $\left(\operatorname{Ind}_{\mathrm{GL}_{2}\left(k_{v}\right)}^{\mathrm{SO}_{4}\left(k_{v}\right)} \pi_{2, v}\right)^{\eta_{v}}$ is fully induced from the Levi factor $\mathrm{GL}_{1}\left(k_{v}\right) \times \mathrm{GL}_{1}\left(k_{v}\right)$. In other words, $\pi_{1, v} \nu \otimes\left(\operatorname{Ind}_{\mathrm{GL}_{2}\left(k_{v}\right)}^{\mathrm{SO}_{4}\left(k_{v}\right)} \pi_{2, v}\right)^{\eta_{v}}$ is fully induced from a tempered representation of a smaller parabolic subgroup.

In the notation of Lemma 2.9 let $w=w_{1} w_{2} w_{1}$ be the unique nontrivial Weyl group element for $\mathrm{GL}_{2} \times \mathrm{SO}_{4} \subset \mathrm{SO}_{8}$ case and $\underline{s}=1$. The Levi factor $L$ is one of $\mathrm{GL}_{1} \times \mathrm{GL}_{1} \times \mathrm{SO}_{4}, \mathrm{GL}_{2} \times \mathrm{GL}_{1} \times \mathrm{GL}_{1}, \mathrm{GL}_{1} \times \mathrm{GL}_{1} \times \mathrm{GL}_{1} \times \mathrm{GL}_{1}$ and

$$
\underline{s}+\underline{s}^{\prime}=\left\{\begin{array}{l}
\left(1+r_{1}, 1-r_{1}\right), \\
\left(1, r_{2},-r_{2}\right), \\
\left(1+r_{1}, 1-r_{1}, r_{2},-r_{2}\right)
\end{array}\right.
$$

where $0<r_{i}<1 / 2$. Let $w^{\prime}=1$ and in the notation of the proof of Proposition 1.12

$$
w^{\prime \prime}=\left\{\begin{array}{l}
(1,2) \\
(3,4) w_{2} \\
(1,2)(3,4) w_{2} .
\end{array}\right.
$$

Then $w^{\prime-1}\left(\underline{s}+\underline{s}^{\prime}\right)$ in the first case is in the positive Weyl chamber, while in the remaining two cases it satisfies the inequalities of Lemma 2.9. The Weyl group element $w^{\prime \prime} w w^{\prime}$ is the longest element of the Weyl group of $\left(\mathrm{SO}_{8}, L\right)$ and the normalized intertwining operator $N\left(w\left(\underline{s}+\underline{s}^{\prime}\right), w\left(\tau_{v}\right), w^{\prime \prime}\right)=$ $N\left(w(\underline{s}), w\left(\pi_{v}^{\prime}\right), w^{\prime \prime}\right)$ is an isomorphism. Therefore, by Lemma $2.9, \Pi_{v}^{\prime \eta_{v}}$ is irreducible. Observe that in the second case if $\pi_{1, v}$ is not square-integrable, it is 
fully induced from a square-integrable representation of $\mathrm{GL}_{1}\left(k_{v}\right) \times \mathrm{GL}_{1}\left(k_{v}\right)$ and replacing $\underline{s}+\underline{s}^{\prime}$ by $\left(1,1, r_{2},-r_{2}\right)$ and $w^{\prime \prime}$ by $(1,2)(3,4) w_{2}$ the claim still holds.

Before giving the decomposition of the space $L_{A_{3}}^{2}$ we give a few facts concerning the induced representation $\operatorname{Ind}_{\mathrm{GL}_{1}^{\prime}\left(k_{v}\right) \times \mathrm{GL}_{1}^{\prime}\left(k_{v}\right)}^{\mathrm{GL}^{\prime}\left(k_{v}\right)}\left(\pi_{1, v}^{\prime} \otimes \pi_{2, v}^{\prime}\right)$, where $\pi_{i, v}^{\prime}$ are the irreducible unitary representations with trivial central characters and the normalized intertwining operator $N\left(0, \pi_{1, v}^{\prime} \otimes \pi_{2, v}^{\prime}, w_{1}\right)$, where $w_{1}$ is the nontrivial Weyl group element of the maximal parabolic subgroup case $\mathrm{GL}_{1}^{\prime} \times \mathrm{GL}_{1}^{\prime} \subset \mathrm{GL}_{2}^{\prime}$. By Theorem 4.2 of [6] at split non-archimedean, Theorem B.2.d of [8] at non-split and results of [47] at archimedean places, the induced representation above is irreducible and hence the normalized operator $N\left(0, \pi_{1, v}^{\prime} \otimes \pi_{2, v}^{\prime}, w_{1}\right)$ acts as $\pm I d_{v}$. Let $\eta_{v}$ denote the sign.

THEOREM 2.18. The subspace $L_{A_{3}}^{2}$ of the residual spectrum of $G_{2}^{\prime}(\mathbb{A})$ decomposes into

$$
L_{A_{3}}^{2}=\left(\oplus_{\pi^{\prime}} \mathcal{A}_{3}^{(1)}\left(\pi^{\prime}\right)\right) \oplus\left(\oplus_{\pi^{\prime}} \mathcal{A}_{3}^{(2)}\left(\pi^{\prime}\right)\right) .
$$

The former sum is over all the cuspidal automorphic representations $\pi^{\prime} \cong$ $\pi_{1}^{\prime} \otimes \pi_{2}^{\prime}$ of $M_{0}^{\prime}(\mathbb{A})$ such that the central characters $\omega_{\pi_{1}^{\prime}}=\omega_{\pi_{2}^{\prime}}$ are trivial, $\pi_{1}^{\prime} ¥ \pi_{2}^{\prime}$ and the parity condition $\prod_{v} \eta_{v}=1$ holds. The latter sum is over all the cuspidal automorphic representations $\pi^{\prime} \cong \pi_{1}^{\prime} \otimes \pi_{2}^{\prime}$ of $M_{0}^{\prime}(\mathbb{A})$ such that the central characters $\omega_{\pi_{1}^{\prime}}=\omega_{\pi_{2}^{\prime}}$ are trivial, $\pi_{1}^{\prime} \cong \pi_{2}^{\prime}$ and the parity condition $\prod_{v} \eta_{v}=-1$ holds.

$\mathcal{A}_{3}^{(1)}\left(\pi^{\prime}\right)$ is the irreducible space of automorphic forms spanned by the iterated residue

$$
\operatorname{Res}_{z=1 / 2} \operatorname{Res}_{2 s_{2}=1} E\left(\underline{s}, g ; f_{\underline{s}}, \pi^{\prime}\right)
$$

at $\underline{s}=(1 / 2,1 / 2)$ of the Eisenstein series attached to $\pi^{\prime} \cdot \mathcal{A}_{3}^{(2)}\left(\pi^{\prime}\right)$ is the irreducible space of automorphic forms spanned by the iterated residue

$$
\operatorname{Res}_{z=1 / 2} \operatorname{Res}_{2 s_{2}=1}\left(s_{1}-1 / 2\right) E\left(\underline{s}, g ; f_{\underline{s}}, \pi^{\prime}\right)
$$

at $\underline{s}=(1 / 2,1 / 2)$ of the Eisenstein series attached to $\pi^{\prime}$ multiplied by $\left(s_{1}-1 / 2\right)$. For both spaces, by (2.8), the constant term map induces an isomorphism with the image of the normalized intertwining operator

$$
N\left((1 / 2,1 / 2), \pi^{\prime}, w_{2} w_{1} w_{2}\right) .
$$

Proof. The first step is to look at the pole of the constant term (2.2) of the Eisenstein series along the singular hyperplane $2 s_{2}=1$. From Table 2 the pole along this hyperplane occurs if and only if $\omega_{\pi_{2}}$ is trivial for the intertwining operators corresponding to the Weyl group elements $w_{2}, w_{1} w_{2}$, $w_{2} w_{1} w_{2}$ and $w_{1} w_{2} w_{1} w_{2}$ and it is simple. The residues written in the new variable $z$ on $2 s_{2}=1$, up to the nonzero constant

$$
\operatorname{Res}_{s=1} L\left(s, \omega_{\pi_{2}}\right) L\left(2, \omega_{\pi_{2}}\right)^{-1} \varepsilon\left(1, \omega_{\pi_{2}}\right)^{-1},
$$


are given in Table 4.

\begin{tabular}{|c|c|}
\hline$w$ & $\operatorname{Res}_{2 s_{2}=1} r\left(\underline{s}, \pi^{\prime}, w\right)$ \\
\hline \hline$w_{2}$ & 1 \\
\hline \hline$w_{1} w_{2}$ & $\frac{L\left(z+1 / 2, \pi_{1} \times \pi_{2}\right)}{L\left(z+3 / 2, \pi_{1} \times \pi_{2}\right) \varepsilon\left(z+1 / 2, \pi_{1} \times \pi_{2}\right)}$ \\
\hline \hline$w_{2} w_{1} w_{2}$ & $\frac{L\left(z+1 / 2, \pi_{1} \times \pi_{2}\right)}{L\left(z+3 / 2, \pi_{1} \times \pi_{2}\right) \varepsilon\left(z+1 / 2, \pi_{1} \times \pi_{2}\right)} \frac{L\left(2 z, \omega_{\pi_{1}}\right)}{L\left(1+2 z, \omega_{\pi_{1}}\right) \varepsilon\left(2 z, \omega_{\pi_{1}}\right)}$ \\
\hline \hline$w_{1} w_{2} w_{1} w_{2}$ & $\frac{L\left(z+1 / 2, \pi_{1} \times \pi_{2}\right)}{L\left(z+3 / 2, \pi_{1} \times \pi_{2}\right) \varepsilon\left(z+1 / 2, \pi_{1} \times \pi_{2}\right)}$ \\
& $\frac{L\left(z-1 / 2, \pi_{1} \times \pi_{2}\right)}{L\left(z+1 / 2, \pi_{1} \times \pi_{2}\right) \varepsilon\left(z-1 / 2, \pi_{1} \times \pi_{2}\right)} \frac{L\left(2 z, \omega_{\pi_{1}}\right)}{L\left(1+2 z, \omega_{\pi_{1}}\right) \varepsilon\left(2 z, \omega_{\pi_{1}}\right)}$ \\
\hline
\end{tabular}

TABLE 4. Residues along $2 s_{2}=1$ of case A normalizing factors

We are interested in the pole of the four terms in Table 4 at $z=1 / 2$. Observe that since $\omega_{\pi_{2}}$ is trivial, $\pi_{2}$ is selfcontragredient. The conditions $\pi_{1} \cong \pi_{2}$ and $\pi_{1} \cong \tilde{\pi}_{2}$ are equivalent and imply that $\omega_{\pi_{1}}$ is trivial. Hence, by the analytic properties of the $\mathrm{L}$-functions involved, in order to have the pole at $z=1 / 2$ the central character $\omega_{\pi_{1}}$ has to be trivial. Assuming that $\omega_{\pi_{1}}=\omega_{\pi_{2}}$ are trivial we consider the following two cases.

First, let $\pi_{1} \neq \pi_{2}$. Then the pole at $z=1 / 2$ occurs for the intertwining operators corresponding to the Weyl group elements $w_{2} w_{1} w_{2}$ and $w_{1} w_{2} w_{1} w_{2}$ and it is simple. Using the selfcontragredience of $\pi_{1}$ and $\pi_{2}$, by Lemma 2.8 the residues are, up to the nonzero constant

$$
\frac{L\left(1, \pi_{1} \times \pi_{2}\right) \operatorname{Res}_{s=1} L\left(s, \omega_{\pi_{1}}\right)}{L\left(2, \pi_{1} \times \pi_{2}\right) \varepsilon\left(1, \pi_{1} \times \pi_{2}\right) L\left(2, \omega_{\pi_{1}}\right) \varepsilon\left(1, \omega_{\pi_{1}}\right)},
$$

equal to $N\left((1 / 2,1 / 2), \pi^{\prime}, w_{2} w_{1} w_{2}\right)$ and $N\left((1 / 2,1 / 2), \pi^{\prime}, w_{1} w_{2} w_{1} w_{2}\right)$. As $w_{1} w_{2} w_{1} w_{2}=w_{2} w_{1} w_{2} w_{1}$, we write the residue acting on $\otimes_{v} f_{v}$ as

$$
\otimes_{v} N\left((1 / 2,1 / 2), \pi_{v}^{\prime}, w_{2} w_{1} w_{2}\right)\left[\otimes_{v} f_{v}+\otimes_{v} N\left((1 / 2,1 / 2), \pi_{v}^{\prime}, w_{1}\right) f_{v}\right],
$$

where the intertwining operator $N\left((1 / 2,1 / 2), \pi_{v}^{\prime}, w_{1}\right)$ is actually the operator for the group $\mathrm{GL}_{2}^{\prime}\left(k_{v}\right)$. By the discussion preceding the statement of the Theorem, it acts as $\eta_{v} I d_{v}$ at every place. The residue is nonzero if $\prod_{v} \eta_{v}=1$ as claimed. Since $w_{2} w_{1} w_{2} w_{1}(1 / 2,1 / 2)=w_{2} w_{1} w_{2}(1 / 2,1 / 2)=(-1 / 2,-1 / 2)$, the square integrability follows from Lemma 2.7. Therefore, the iterated residue of the constant term of the Eisenstein series is, up to a nonzero constant, equal to the normalized intertwining operator $N\left((1 / 2,1 / 2), \pi^{\prime}, w_{2} w_{1} w_{2}\right)$.

Next, let $\pi_{1} \cong \pi_{2}$. Then the term corresponding to $w_{1} w_{2}$ has the simple pole, while the terms corresponding to $w_{2} w_{1} w_{2}$ and $w_{1} w_{2} w_{1} w_{2}$ have the double pole at $z=1 / 2$. Hence, in order to find the contribution to the residual spectrum, we must calculate the coefficient of $(z-1 / 2)^{-2}$ in the Laurent expansion of $\operatorname{Res}_{2 s_{2}=1} E_{0}\left(\underline{s}, g ; f_{\underline{s}}, \pi^{\prime}\right)$, i.e.

$$
\operatorname{Res}_{z=1 / 2} \operatorname{Res}_{2 s_{2}=1}(z-1 / 2) E_{0}\left(\underline{s}, g ; f_{\underline{s}}, \pi^{\prime}\right) .
$$


Using Table 4, Remark 2.11 and Lemma 2.8, up to the nonzero constant

$$
\frac{\operatorname{Res}_{s=1} L\left(s, \pi_{1} \times \pi_{2}\right) \operatorname{Res}_{s=1} L(s, \mathbf{1})}{L\left(2, \pi_{1} \times \pi_{2}\right) \varepsilon\left(1, \pi_{1} \times \pi_{2}\right) L(2, \mathbf{1}) \varepsilon(1, \mathbf{1})},
$$

where 1 is the trivial character of $\mathbb{A}^{\times} / k^{\times}$, it equals

$$
N\left((1 / 2,1 / 2), \pi^{\prime}, w_{2} w_{1} w_{2}\right)-N\left((1 / 2,1 / 2), \pi^{\prime}, w_{1} w_{2} w_{1} w_{2}\right) .
$$

As in the previous case, decomposing the normalized intertwining operators and using the discussion preceding the statement of the Theorem gives

$$
\left(1-\prod_{v} \eta_{v}\right) N\left((1 / 2,1 / 2), \pi^{\prime}, w_{2} w_{1} w_{2}\right)
$$

Therefore, if $\prod_{v} \eta_{v}=-1$ we obtain the contribution to the residual spectrum as claimed. Up to a nonzero constant, the residue equals the intertwining operator $N\left((1 / 2,1 / 2), \pi^{\prime}, w_{2} w_{1} w_{2}\right)$. The square-integrability criterion is satisfied as in the previous case.

Otherwise, if $\prod_{v} \eta_{v}=1$, the double pole cancels. We are left with the simple pole at $z=1 / 2$ of the terms corresponding to $w_{1} w_{2}, w_{2} w_{1} w_{2}$ and $w_{1} w_{2} w_{1} w_{2}$. But $w_{1} w_{2}(1 / 2,1 / 2)=(-1 / 2,1 / 2)$ and the Langlands square integrability criterion of Lemma 2.7 fails. Therefore, the only possibility for the contribution to the residual spectrum is to have a constituent of the induced representation $\operatorname{Ind}_{\mathrm{GL}}^{G_{1}^{\prime}(\mathbb{A}) \times \mathrm{GL}_{1}^{\prime}(\mathbb{A})}\left(\nu^{1 / 2} \pi_{1}^{\prime} \otimes \nu^{1 / 2} \pi_{2}^{\prime}\right)$ such that the residue at $z=1 / 2$ of the term corresponding to $w_{1} w_{2}$ vanishes. Let $f$ be the automorphic form such that

$$
N\left((1 / 2,1 / 2), \pi^{\prime}, w_{1} w_{2}\right) f=0 .
$$

Now, for $f$ as above, we must calculate the residue at $z=1 / 2$ of the sum of the last two terms in the expression for the constant term of the Eisenstein series. Since $\pi_{1} \cong \pi_{2}$ are selfcontragredient and by the global functional equation

$$
L\left(z-1 / 2, \pi_{1} \times \pi_{2}\right)=\varepsilon\left(z-1 / 2, \pi_{1} \times \pi_{2}\right) L\left(3 / 2-z, \pi_{1} \times \pi_{2}\right),
$$

the last term becomes

$$
\begin{aligned}
& \frac{L\left(3 / 2-z, \pi_{1} \times \pi_{2}\right)}{L\left(z+3 / 2, \pi_{1} \times \pi_{2}\right) \varepsilon\left(z+1 / 2, \pi_{1} \times \pi_{2}\right)} \frac{L\left(2 z, \omega_{\pi_{1}}\right)}{L\left(1+2 z, \omega_{\pi_{1}}\right) \varepsilon\left(2 z, \omega_{\pi_{1}}\right)} \\
& N\left((z, 1 / 2), \pi^{\prime}, w_{2} w_{1} w_{2} w_{1}\right) .
\end{aligned}
$$

The third term is

$$
\frac{L\left(z+1 / 2, \pi_{1} \times \pi_{2}\right)}{L\left(z+3 / 2, \pi_{1} \times \pi_{2}\right) \varepsilon\left(z+1 / 2, \pi_{1} \times \pi_{2}\right)} \frac{L\left(2 z, \omega_{\pi_{1}}\right)}{L\left(1+2 z, \omega_{\pi_{1}}\right) \varepsilon\left(2 z, \omega_{\pi_{1}}\right)}
$$

$$
N\left((z, 1 / 2), \pi^{\prime}, w_{2} w_{1} w_{2}\right) .
$$

Let $a_{-1}$ be the residue at $z=1 / 2$ of the fraction

$$
L\left(z+1 / 2, \pi_{1} \times \pi_{2}\right) L\left(z+3 / 2, \pi_{1} \times \pi_{2}\right)^{-1} \varepsilon\left(z+1 / 2, \pi_{1} \times \pi_{2}\right)^{-1} .
$$


Then the residue of

$$
L\left(3 / 2-z, \pi_{1} \times \pi_{2}\right) L\left(z+3 / 2, \pi_{1} \times \pi_{2}\right)^{-1} \varepsilon\left(z+1 / 2, \pi_{1} \times \pi_{2}\right)^{-1}
$$

is $-a_{-1}$. Let $b_{-1}$ be the residue at $z=1 / 2$ of the fraction

$$
L\left(2 z, \omega_{\pi_{1}}\right) L\left(1+2 z, \omega_{\pi_{1}}\right)^{-1} \varepsilon\left(2 z, \omega_{\pi_{1}}\right)^{-1} .
$$

Let $f_{z}$ be the section of the induced representation $I\left((z, 1 / 2), \pi^{\prime}\right)$ such that $f_{1 / 2}=f$. Then the Taylor expansion around $1 / 2$ of $f_{z}$ equals

$$
f_{z}=f_{1 / 2}+(z-1 / 2) D f_{z}+\ldots
$$

where $D f_{z}$ is the derivative of $f_{z}$ as a function of $z$ at $z=1 / 2$.

The normalized intertwining operators as functions of $z$ have their Taylor expansions around $z=1 / 2$ as follows

$$
\begin{aligned}
N\left((z, 1 / 2), \pi^{\prime}, w_{2} w_{1} w_{2}\right)= & N\left((1 / 2,1 / 2), \pi^{\prime}, w_{2} w_{1} w_{2}\right) \\
& +(z-1 / 2) D N\left((z, 1 / 2), \pi^{\prime}, w_{2} w_{1} w_{2}\right)+\cdots \\
N\left((-1 / 2,-z), \pi^{\prime}, w_{1}\right)= & N\left((-1 / 2,-1 / 2), \pi^{\prime}, w_{1}\right) \\
& +(z-1 / 2) D N\left((-1 / 2,-z), \pi^{\prime}, w_{1}\right)+\cdots
\end{aligned}
$$

where $D N(\cdot)$ denotes the derivatives at $z=1 / 2$ of the corresponding normalized intertwining operators. By the discussion preceding the statement of the Theorem, the normalized intertwining operator $N\left((-1 / 2,-1 / 2), \pi_{1}^{\prime} \otimes \pi_{2}^{\prime}, w_{1}\right)$ acts as $\left(\prod_{v} \eta_{v}\right) I d$. In this case $\prod_{v} \eta_{v}=1$ and $N\left((-1 / 2,-1 / 2), \pi^{\prime}, w_{1}\right)$ is the identity. Hence, by the decomposition of the intertwining operators

$$
\begin{aligned}
& N\left((z, 1 / 2), \pi^{\prime}, w_{1} w_{2} w_{1} w_{2}\right)=N\left((1 / 2,1 / 2), \pi^{\prime}, w_{2} w_{1} w_{2}\right) \\
& \quad+(z-1 / 2) \cdot\left[D N\left((-1 / 2,-z), \pi^{\prime}, w_{1}\right) N\left((1 / 2,1 / 2), \pi^{\prime}, w_{2} w_{1} w_{2}\right)\right. \\
& \left.\quad+D N\left((z, 1 / 2), \pi^{\prime}, w_{2} w_{1} w_{2}\right)\right]+\cdots
\end{aligned}
$$

The constant terms of the Taylor expansions around $z=1 / 2$ of both intertwining operators applied to $f_{1 / 2}$ are zero by the assumption on $f=f_{1 / 2}$ because

$$
\begin{aligned}
& N\left((1 / 2,1 / 2), \pi_{v}^{\prime}, w_{2} w_{1} w_{2}\right) f \\
& \quad=N\left((-1 / 2,1 / 2), w_{1} w_{2}\left(\pi_{v}^{\prime}\right), w_{2}\right) N\left((1 / 2,1 / 2), \pi_{v}^{\prime}, w_{1} w_{2}\right) f=0 .
\end{aligned}
$$

Therefore, by (2.21) and (2.22), the residue at $z=1 / 2$ of term (2.20) is the sum of

$$
\left\{\begin{array}{l}
a_{-1} b_{-1} D N\left((z, 1 / 2), \pi^{\prime}, w_{2} w_{1} w_{2}\right) f_{1 / 2} \\
a_{-1} b_{-1} N\left((1 / 2,1 / 2), \pi^{\prime}, w_{2} w_{1} w_{2}\right) D f_{z}
\end{array}\right.
$$

while by (2.21) and (2.23) the residue at $z=1 / 2$ of term (2.19) is the sum of

$$
\left\{\begin{array}{l}
-a_{-1} b_{-1} D N\left((-1 / 2,-z), \pi^{\prime}, w_{1}\right) N\left((1 / 2,1 / 2), \pi^{\prime}, w_{2} w_{1} w_{2}\right) f_{1 / 2}, \\
-a_{-1} b_{-1} D N\left((z, 1 / 2), \pi^{\prime}, w_{2} w_{1} w_{2}\right) f_{1 / 2} \\
-a_{-1} b_{-1} N\left((1 / 2,1 / 2), \pi^{\prime}, w_{2} w_{1} w_{2}\right) D f_{z} .
\end{array}\right.
$$


After the addition of the above residues, all the terms cancel except

$$
-a_{-1} b_{-1} D N\left((-1 / 2,-z), \pi^{\prime}, w_{1}\right) N\left((1 / 2,1 / 2), \pi^{\prime}, w_{2} w_{1} w_{2}\right) f_{1 / 2}
$$

which is zero by the assumption on $f_{1 / 2}$ as above. Thus, in the case $\pi_{1} \cong \pi_{2}$ and $\prod_{v} \eta_{v}=1$ there is no contribution to the residual spectrum.

It remains to prove the irreducibility of the image of the normalized operator $N\left((1 / 2,1 / 2), \pi^{\prime}, w_{2} w_{1} w_{2}\right)$. Using Lemma 2.9 we prove the irreducibility of the image of the local operators $N\left((1 / 2,1 / 2), \pi_{v}^{\prime}, w_{2} w_{1} w_{2}\right)$ at every place $v$. Since

$$
N\left(w_{2} w_{1} w_{2}(1 / 2,1 / 2), w_{2} w_{1} w_{2}\left(\pi_{v}^{\prime}\right), w_{1}\right)=\eta_{v} I d_{v},
$$

it is isomorphic to the image of $N\left((1 / 2,1 / 2), \pi_{v}^{\prime}, w_{1} w_{2} w_{1} w_{2}\right)$. Let $w=$ $w_{1} w_{2} w_{1} w_{2}$ and $\underline{s}=(1 / 2,1 / 2)$. If $\pi_{i, v}^{\prime}$ are both square-integrable, then by the Langlands classification the image is irreducible since $w$ is the longest Weyl group element for $\left(G_{2}^{\prime}, M_{0}^{\prime}\right)$. Observe that this is the case at all nonsplit places. form

Otherwise, at least one of the representations $\pi_{i, v}$ of $\mathrm{GL}_{2}\left(k_{v}\right)$ is of the

$$
\pi_{i, v} \cong \operatorname{Ind}_{\mathrm{GL}_{1}\left(k_{v}\right) \times \mathrm{GL}_{1}\left(k_{v}\right)}^{\mathrm{GL}_{2}\left(k_{v}\right)}\left(\mu_{i, v}|\cdot|^{r_{i}} \otimes \mu_{i, v}^{\prime}|\cdot|^{-r_{i}}\right),
$$

where $0 \leqslant r_{i}<1 / 2$ and $\mu_{i, v}=\mu_{i, v}^{\prime}$ if $r_{i} \neq 0$. Then, $\pi_{v}$ is the fully induced representation from a square-integrable representation $\tau_{v}$ of the Levi factor of a smaller parabolic subgroup. In the notation of Lemma $2.9, L$ is one of the Levi factors $\mathrm{GL}_{1} \times \mathrm{GL}_{1} \times \mathrm{GL}_{2}, \mathrm{GL}_{2} \times \mathrm{GL}_{1} \times \mathrm{GL}_{1}, \mathrm{GL}_{1} \times \mathrm{GL}_{1} \times \mathrm{GL}_{1} \times \mathrm{GL}_{1}$ and

$$
\underline{s}+\underline{s}^{\prime}=\left\{\begin{array}{l}
\left(1 / 2+r_{1}, 1 / 2-r_{1}, 1 / 2\right), \\
\left(1 / 2,1 / 2+r_{2}, 1 / 2-r_{2}\right), \\
\left(1 / 2+r_{1}, 1 / 2-r_{1}, 1 / 2+r_{2}, 1 / 2-r_{2}\right),
\end{array}\right.
$$

where $0 \leqslant r_{i}<1 / 2$. Assume $r_{1} \geqslant r_{2}$. The other case is settled in the same way. In the notation as in the proof of Proposition 1.12, let

$$
w^{\prime}=\left\{\begin{array}{l}
(1)(2,3) \\
(1,2)(3) \\
(1)(2,3,4)
\end{array}\right.
$$

Then $w^{\prime-1}\left(\underline{s}+\underline{s}^{\prime}\right)$ satisfies inequality (2.10) of Lemma 2.9 and $N\left(w^{\prime-1}(\underline{s}+\right.$ $\left.\left.\underline{s}^{\prime}\right), w^{\prime-1}\left(\tau_{v}\right), w^{\prime}\right)$ is an isomorphism onto $I\left(\underline{s}+\underline{s}^{\prime}, \tau_{v}\right) \cong I\left(\underline{s}, \pi_{v}\right)$. Let

$$
w^{\prime \prime}=\left\{\begin{array}{l}
(1,3,2) \\
(1,2,3) \\
(1,4,2)(3)
\end{array}\right.
$$

Then $w^{\prime \prime} w w^{\prime}$ is the longest element of the Weyl group for $\left(\mathrm{SO}_{8}, w^{\prime-1}(L)\right)$ and $N\left(w(\underline{s}), w\left(\pi_{v}\right), w^{\prime \prime}\right)=N\left(w\left(\underline{s}+\underline{s}^{\prime}\right), w\left(\tau_{v}\right), w^{\prime \prime}\right)$ is an isomorphism. Therefore, by Lemma 2.9 , the image of the normalized operator $N\left((1 / 2,1 / 2), \pi_{v}^{\prime}, w\right)$ is irreducible. 
REMARK 2.24. This is the only case where the question of the multiplicities in the decomposition is left open. Namely, the second part of the decomposition of $L_{A_{3}}^{2}$ may not be multiplicity one because the iterated pole of the Eisenstein series is not simple. In order to find the multiplicities we should decompose the scalar product of the pseudo-Eisenstein series.

2.3. Case $B$. In this case one of the global lifts $\pi_{1}$ and $\pi_{2}$ is a residual and the other is a cuspidal automorphic representation of $\mathrm{GL}_{2}(\mathbb{A})$. In the calculation we assume that $\pi_{1}$ is residual. The other case is the same. So, $\pi_{1}^{\prime}=\chi_{1} \circ \operatorname{det}^{\prime}$ is one-dimensional and its global lift is $\pi_{1}=\chi_{1} \circ$ det. By our definition at non-split places, the local lift of $\chi_{1, v} \circ \operatorname{det}_{v}^{\prime}$ is the Steinberg representation $S t_{\chi_{1, v}}$ of $\mathrm{GL}_{2}\left(k_{v}\right)$ which is not the local component of $\pi_{1}$ at $v$.

The local normalizing factors, obtained in the previous Section, at the split places are given by (1.9) and the maximal parabolic subgroup cases by (1.13) and (1.11), while at the non-split places they are given using the local lifts by (1.16) and the maximal parabolic cases by (1.18) and (1.17). Since the central characters of $\chi_{1, v} \circ \operatorname{det}_{v}$ and $S t_{\chi_{1, v}}$ are both equal to $\chi_{1, v}^{2}$ and $\pi_{2}$ is cuspidal automorphic, the products over all places of the local Hecke $\mathrm{L}$-functions and $\varepsilon$-factors appearing in the local normalizing factors for the maximal parabolic case $\mathrm{GL}_{1}^{\prime} \subset G_{1}^{\prime}$ give just the global Hecke L-functions and $\varepsilon$-factors of the central characters $\chi_{1}^{2}$ and $\omega_{\pi_{2}}$.

The local Rankin-Selberg L-function and $\varepsilon$-factor appearing in the local normalizing factor at a non-split place $v$ are $L\left(s, S t_{\chi_{1, v}} \times \widetilde{\pi}_{2, v}\right)$ and $\varepsilon\left(s, S t_{\chi_{1, v}} \times\right.$ $\left.\tilde{\pi}_{2, v}, \psi_{v}\right)$. If $\pi_{2, v}$ is a supercuspidal representation of $\mathrm{GL}_{2}\left(k_{v}\right)$, by Theorem (3.1) and Sections 8 and 9 of [20],

$$
L\left(s, S t_{\chi_{1, v}} \times \tilde{\pi}_{2, v}\right)=L\left(s+1 / 2, \chi_{1, v} \tilde{\pi}_{2, v}\right),
$$

$$
\begin{aligned}
& \varepsilon\left(s, \chi_{1, v} \times \tilde{\pi}_{2, v}, \psi_{v}\right) \\
& \quad=\varepsilon\left(s+1 / 2, \chi_{1, v} \widetilde{\pi}_{2, v}, \psi_{v}\right) \varepsilon\left(s-1 / 2, \chi_{1, v} \widetilde{\pi}_{2, v}, \psi_{v}\right) \frac{L\left(1 / 2-s, \chi_{1, v}^{-1} \pi_{2, v}\right)}{L\left(s-1 / 2, \chi_{1, v} \widetilde{\pi}_{2, v}\right)} .
\end{aligned}
$$

By Section (3.1) of [18] the principal L-function of a supercuspidal representation of $\mathrm{GL}_{2}\left(k_{v}\right)$ at a non-archimedean place $v$ is identically equal to 1 . Therefore, we can write these local L-functions and $\varepsilon$-factors as

$$
\begin{gathered}
L\left(s, S t_{\chi_{1, v}} \times \tilde{\pi}_{2, v}\right)=L\left(s+1 / 2, \chi_{1, v} \tilde{\pi}_{2, v}\right) L\left(s-1 / 2, \chi_{1, v} \tilde{\pi}_{2, v}\right), \\
\varepsilon\left(s, \chi_{1, v} \times \tilde{\pi}_{2, v}, \psi_{v}\right)=\varepsilon\left(s+1 / 2, \chi_{1, v} \tilde{\pi}_{2, v}, \psi_{v}\right) \varepsilon\left(s-1 / 2, \chi_{1, v} \tilde{\pi}_{2, v}, \psi_{v}\right),
\end{gathered}
$$

to be consistent with the local normalizing factors at split places (1.13).

If not supercuspidal, $\pi_{2, v}$ is a square-integrable representation of $\mathrm{GL}_{2}\left(k_{v}\right)$ because it is the local lift of the supercuspidal representation $\pi_{2, v}^{\prime}$ of $\mathrm{GL}_{1}^{\prime}\left(k_{v}\right)$. Hence, $\pi_{2, v} \cong S t_{\chi_{2, v}}$ for a unitary character $\chi_{2, v}$ of $k_{v}^{\times}$. Again, by Theorem 
(3.1), Sections 8 and 9 of [20] and Section (3.1) of [18]

$$
\begin{aligned}
L\left(s, S t_{\chi_{1, v}} \times \widetilde{\pi}_{2, v}\right)= & L\left(s+1, \chi_{1, v} \chi_{2, v}^{-1}\right) L\left(s, \chi_{1, v} \chi_{2, v}^{-1}\right) \\
= & L\left(s+1 / 2, \chi_{1, v} \widetilde{\pi}_{2, v}\right) L\left(s-1 / 2, \chi_{1, v} \widetilde{\pi}_{2, v}\right), \\
\varepsilon\left(s, S t_{\chi_{1, v}} \times \widetilde{\pi}_{2, v}, \psi_{v}\right) & \varepsilon\left(s+1, \chi_{1, v} \chi_{2, v}^{-1}, \psi_{v}\right) \varepsilon\left(s, \chi_{1, v} \chi_{2, v}^{-1}, \psi_{v}\right)^{2} \varepsilon\left(s-1, \chi_{1, v} \chi_{2, v}^{-1}, \psi_{v}\right) \\
= & \cdot \frac{L\left(-s, \chi_{1, v}^{-1} \chi_{2, v}\right)}{L\left(s, \chi_{1, v} \chi_{2, v}^{-1}\right)} \frac{L\left(1-s, \chi_{1, v}^{-1} \chi_{2, v}\right)}{L\left(s-1, \chi_{1, v} \chi_{2, v}^{-1}\right)} \\
= & \varepsilon\left(s+1 / 2, \chi_{1, v} \widetilde{\pi}_{2, v}, \psi_{v}\right) \varepsilon\left(s-1 / 2, \chi_{1, v} \widetilde{\pi}_{2, v}, \psi_{v}\right),
\end{aligned}
$$

which is consistent with the local normalizing factors at split places (1.13).

Therefore, in the global normalizing factor, obtained as the product over all places of these local principal $\mathrm{L}$-functions and $\varepsilon$-factors for $\mathrm{GL}_{2}$, the global

\begin{tabular}{|c|c|}
\hline$w$ & $r\left(\underline{s}, \pi^{\prime}, w\right)$ \\
\hline 1 & 1 \\
\hline$w_{1}$ & $\frac{L\left(s_{1}-s_{2}-1 / 2, \chi_{1} \tilde{\pi}_{2}\right)}{\overline{L\left(s_{1}-s_{2}+3 / 2, \chi_{1} \tilde{\pi}_{2}\right) \varepsilon\left(s_{1}-s_{2}-1 / 2, \chi_{1} \tilde{\pi}_{2}\right) \varepsilon\left(s_{1}-s_{2}+1 / 2, \chi_{1} \tilde{\pi}_{2}\right)}}$ \\
\hline$w_{2}$ & $\frac{L\left(2 s_{2}, \omega_{\pi_{2}}\right)}{L\left(1+2 s_{2}, \omega_{\pi_{2}}\right) \varepsilon\left(2 s_{2}, \omega_{\pi_{2}}\right)}$ \\
\hline$w_{1} w_{2}$ & $\frac{L\left(2 s_{2}, \omega_{\pi_{2}}\right)}{L\left(1+2 s_{2}, \omega_{\pi_{2}}\right) \varepsilon\left(2 s_{2}, \omega_{\pi_{2}}\right)} \frac{L\left(s_{1}+s_{2}-1 / 2, \chi_{1} \pi_{2}\right)}{L\left(s_{1}+s_{2}+3 / 2, \chi_{1} \pi_{2}\right) \varepsilon\left(s_{1}+s_{2}-1 / 2, \chi_{1} \pi_{2}\right) \varepsilon\left(s_{1}+s_{2}+1 / 2, \chi_{1} \pi_{2}\right)}$ \\
\hline$w_{2} w_{1}$ & $\frac{L\left(s_{1}-s_{2}-1 / 2, \chi_{1} \tilde{\pi}_{2}\right)}{L\left(s_{1}-s_{2}+3 / 2, \chi_{1} \widetilde{\pi}_{2}\right) \varepsilon\left(s_{1}-s_{2}-1 / 2, \chi_{1} \widetilde{\pi}_{2}\right) \varepsilon\left(s_{1}-s_{2}+1 / 2, \chi_{1} \tilde{\pi}_{2}\right)} \frac{L\left(2 s_{1}, \chi_{1}^{2}\right)}{L\left(1+2 s_{1}, \chi_{1}^{2}\right) \varepsilon\left(2 s_{1}, \chi_{1}^{2}\right)}$ \\
\hline$w_{1} w_{2} w_{1}$ & $\begin{array}{c}L\left(s_{1}-s_{2}-1 / 2, \chi_{1} \tilde{\pi}_{2}\right) \\
\frac{L\left(s_{1}-s_{2}+3 / 2, \chi_{1} \tilde{\pi}_{2}\right) \varepsilon\left(s_{1}-s_{2}-1 / 2, \chi_{1} \tilde{\pi}_{2}\right) \varepsilon\left(s_{1}-s_{2}+1 / 2, \chi_{1} \tilde{\pi}_{2}\right)}{L\left(2 s_{1}, \chi_{1}^{2}\right)} \\
\frac{L\left(s_{1}+s_{2}-1 / 2, \chi_{1} \pi_{2}\right)}{L\left(1+2 s_{1}, \chi_{1}^{2}\right) \varepsilon\left(2 s_{1}, \chi_{1}^{2}\right)} \frac{L\left(s_{1}+s_{2}+3 / 2, \chi_{1} \pi_{2}\right) \varepsilon\left(s_{1}+s_{2}-1 / 2, \chi_{1} \pi_{2}\right) \varepsilon\left(s_{1}+s_{2}+1 / 2, \chi_{1} \pi_{2}\right)}{L}\end{array}$ \\
\hline$w_{2} w_{1} w_{2}$ & $\begin{array}{c}\frac{L\left(2 s_{2}, \omega_{\pi_{2}}\right)}{L\left(1+2 s_{2}, \omega_{\pi_{2}}\right) \varepsilon\left(2 s_{2}, \omega_{\pi_{2}}\right)} \\
\frac{L\left(s_{1}+s_{2}-1 / 2, \chi_{1} \pi_{2}\right)}{L\left(s_{1}+s_{2}+3 / 2, \chi_{1} \pi_{2}\right) \varepsilon\left(s_{1}+s_{2}-1 / 2, \chi_{1} \pi_{2}\right) \varepsilon\left(s_{1}+s_{2}+1 / 2, \chi_{1} \pi_{2}\right)} \frac{L\left(2 s_{1}, \chi_{1}^{2}\right)}{L\left(1+2 s_{1}, \chi_{1}^{2}\right) \varepsilon\left(2 s_{1}, \chi_{1}^{2}\right)}\end{array}$ \\
\hline$w_{1} w_{2} w_{1} w_{2}$ & 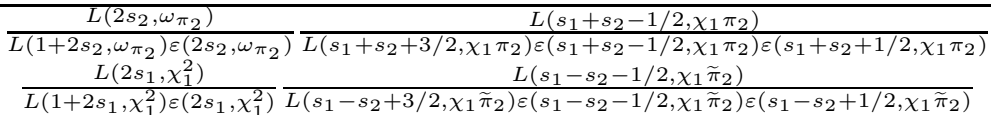 \\
\hline
\end{tabular}
principal L-function and $\varepsilon$-factor of the cuspidal automorphic representation of $\mathrm{GL}_{2}(\mathbb{A})$ appear. All the global normalizing factors in this case are given in Table 5. The analytic properties of the $\mathrm{L}$-functions involved are given in Remark 1.12 and show that the singular hyperplanes of the constant term of the Eisenstein series in (2.1) are as given in Figure 2.

TABLE 5. Case B normalizing factors of $A\left(\underline{s}, \pi^{\prime}, w\right)$ for $w \in W\left(M_{0}^{\prime}\right)$

While deforming the line of integration in (2.1) from $\underline{s}_{0}$ to the origin of $\mathfrak{a}_{M_{0}^{\prime}, \mathbb{C}}^{*}$ inside the positive Weyl chamber as in Figure 2, we cross the singular hyperplanes. Taking the coordinate systems on those hyperplanes gives the points where the possible poles of $(2.2)$ occur. In this case there is just one 


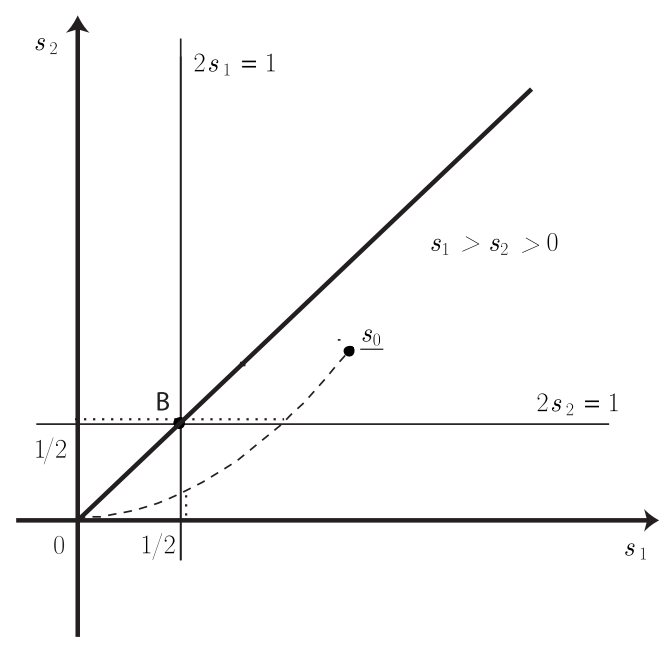

Figure 2. Case B singular hyperplanes

such point $B(1 / 2,1 / 2)$ in Figure 2 . It lies on the singular hyperplane $2 s_{2}=1$. For $2 s_{2}=1$ the coordinate system is given by $s_{1}=z$ and $s_{2}=1 / 2$, where $z$ is the new coordinate. Point $B$ corresponds to $z=1 / 2$. Then, the space $L_{B}^{2}$ is obtained as the iterated residue

$$
\operatorname{Res}_{z=1 / 2} \operatorname{Res}_{2 s_{2}=1} E\left(\underline{s}, g ; f_{\underline{s}}, \pi^{\prime}\right),
$$

of the Eisenstein series attached to the case B cuspidal automorphic representations $\pi^{\prime}$ of $M_{0}^{\prime}(\mathbb{A})$.

Before giving the decomposition of the case $\mathrm{B}$ contribution $L_{B}^{2}$ to the residual spectrum, we introduce some notation. Consider the local $\mathrm{GL}_{2}^{\prime}-$ intertwining operator $N\left(0,\left(\chi_{1, v} \circ \operatorname{det}_{v}^{\prime}\right) \otimes \pi_{2, v}^{\prime}, w_{1}\right)$ acting on the induced representation $\operatorname{Ind}_{\mathrm{GL}_{1}^{\prime}\left(k_{v}\right) \times \mathrm{GL}_{1}^{\prime}\left(k_{v}\right)}^{\mathrm{GL}_{2}^{\prime}\left(k_{v}\right)}\left(\left(\chi_{1, v} \circ \operatorname{det}_{v}^{\prime}\right) \otimes \pi_{2, v}^{\prime}\right)$. By Theorem 4.2 of [6] at split non-archimedean, Theorem B.2.d of [8] at non-split and results of [47] at archimedean places, the induced representation is irreducible. Hence, the normalized operator acts as $\pm I d_{v}$. Denote the sign by $\eta_{v}$. Abusing the notation the sign of $N\left(0, \pi_{1, v}^{\prime} \otimes\left(\chi_{2, v} \circ \operatorname{det}_{v}^{\prime}\right), w_{1}\right)$ will also be denoted by $\eta_{v}$.

THEOREM 2.25. The subspace $L_{B}^{2}$ of the residual spectrum of $G_{2}^{\prime}(\mathbb{A})$ decomposes into

$$
L_{B}^{2}=\left(\oplus_{\pi^{\prime}} \mathcal{B}^{(1)}\left(\pi^{\prime}\right)\right) \oplus\left(\oplus_{\pi^{\prime}} \mathcal{B}^{(2)}\left(\pi^{\prime}\right)\right) .
$$

The former sum is over all the cuspidal automorphic representations $\pi^{\prime} \cong$ $\left(\chi_{1} \circ \operatorname{det}^{\prime}\right) \otimes \pi_{2}^{\prime}$ of $M_{0}^{\prime}(\mathbb{A})$ such that $\chi_{1}$ is a quadratic character, $\pi_{2}^{\prime}$ is not onedimensional, the central character of $\pi_{2}^{\prime}$ is trivial, $L\left(1 / 2, \chi_{1} \pi_{2}\right)$ is nonzero and the parity condition $\prod_{v} \eta_{v}=1$ holds. The latter sum is over all the cuspidal 
automorphic representations $\pi^{\prime} \cong \pi_{1}^{\prime} \otimes\left(\chi_{2} \circ \operatorname{det}^{\prime}\right)$ of $M_{0}^{\prime}(\mathbb{A})$ such that $\chi_{2}$ is a quadratic character, $\pi_{1}^{\prime}$ is not one-dimensional, the central character of $\pi_{1}^{\prime}$ is trivial, $L\left(1 / 2, \chi_{2} \pi_{1}\right)$ is nonzero and the parity condition $\prod_{v} \eta_{v}=1$ holds.

Both $\mathcal{B}^{(1)}\left(\pi^{\prime}\right)$ and $\mathcal{B}^{(2)}\left(\pi^{\prime}\right)$ are the irreducible spaces of automorphic forms spanned by the iterated residues

$$
\operatorname{Res}_{z=1 / 2} \operatorname{Res}_{2 s_{2}=1} E\left(\underline{s}, g ; f_{\underline{s}}, \pi^{\prime}\right)
$$

at $\underline{s}=(1 / 2,1 / 2)$ of the Eisenstein series attached to $\pi^{\prime}$. For both spaces, by (2.8), the constant term map induces an isomorphism with the image of the normalized intertwining operator

$$
N\left((1 / 2,1 / 2), \pi^{\prime}, w_{2} w_{1} w_{2}\right) .
$$

Proof. In the proof we obtain the spaces of automorphic forms $\mathcal{B}^{(1)}\left(\pi^{\prime}\right)$. As mentioned at the beginning of this Subsection, spaces $\mathcal{B}^{(2)}\left(\pi^{\prime}\right)$ are obtained in completely the same way.

We must first look at the pole of the constant term of the Eisenstein series along $2 s_{2}=1$. From Table 5 , the pole along $2 s_{2}=1$ occurs if and only if $\omega_{\pi_{2}}$ is trivial. Then it is simple and occurs for the terms corresponding to $w_{2}$, $w_{1} w_{2}, w_{2} w_{1} w_{2}$ and $w_{1} w_{2} w_{1} w_{2}$. The residues of those terms along $2 s_{2}=1 \mathrm{in}$ the new variable $z$ are given in Table 6 up to the nonzero constant

$$
\operatorname{Res}_{s=1} L(s, \mathbf{1}) L(2, \mathbf{1})^{-1} \varepsilon(1, \mathbf{1})^{-1},
$$

where $\mathbf{1}$ is the trivial character of $\mathbb{A}^{\times} / k^{\times}$. Observe that $\pi_{2}$ is selfcontragredient since $\omega_{\pi_{2}}$ is trivial.

\begin{tabular}{|c|c|}
\hline$w$ & $\operatorname{Res}_{2 s_{2}=1} r\left(\underline{s}, \pi^{\prime}, w\right)$ \\
\hline \hline$w_{2}$ & 1 \\
\hline \hline$w_{1} w_{2}$ & $\frac{L\left(z, \chi_{1} \pi_{2}\right)}{L\left(z+2, \chi_{1} \pi_{2}\right) \varepsilon\left(z, \chi_{1} \pi_{2}\right) \varepsilon\left(z+1, \chi_{1} \pi_{2}\right)}$ \\
\hline \hline$w_{2} w_{1} w_{2}$ & $\frac{L\left(z, \chi_{1} \pi_{2}\right)}{L\left(z+2, \chi_{1} \pi_{2}\right) \varepsilon\left(z, \chi_{1} \pi_{2}\right) \varepsilon\left(z+1, \chi_{1} \pi_{2}\right)} \frac{L\left(1+2 z, \chi_{1}^{2}\right) \varepsilon\left(2 z, \chi_{1}^{2}\right)}{L\left(z, \chi_{1} \pi_{2}\right)}$ \\
\hline \hline$w_{1} w_{2} w_{1} w_{2}$ & $\frac{L\left(z+2, \chi_{1} \pi_{2}\right) \varepsilon\left(z, \chi_{1} \pi_{2}\right) \varepsilon\left(z+1, \chi_{1} \pi_{2}\right)}{L\left(2 z, \chi_{1}^{2}\right)}$ \\
& $\frac{L\left(z-1, \chi_{1} \pi_{2}\right)}{L\left(1+2 z, \chi_{1}^{2}\right) \varepsilon\left(2 z, \chi_{1}^{2}\right)} \frac{L\left(z+1, \chi_{1} \pi_{2}\right) \varepsilon\left(z-1, \chi_{1} \pi_{2}\right) \varepsilon\left(z, \chi_{1} \pi_{2}\right)}{L(2)}$ \\
\hline
\end{tabular}

TABLE 6. Residues along $2 s_{2}=1$ of case B normalizing factors

Now, we look at the possible pole at $z=1 / 2$ of the terms in Table 6. By the analytic properties of the $\mathrm{L}$-functions involved, only the terms corresponding to $w_{2} w_{1} w_{2}$ and $w_{1} w_{2} w_{1} w_{2}$ have the simple pole at $z=1 / 2$ if $\chi_{1}$ is a quadratic character. Up to the nonzero constant

$\operatorname{Res}_{s=1} L(s, \mathbf{1}) L(2, \mathbf{1})^{-1} \varepsilon(1, \mathbf{1})^{-1} L\left(5 / 2, \chi_{1} \pi_{2}\right)^{-1} \varepsilon\left(1 / 2, \chi_{1} \pi_{2}\right)^{-1} \varepsilon\left(3 / 2, \chi_{1} \pi_{2}\right)^{-1}$, 
the residues are equal to $L\left(1 / 2, \chi_{1} \pi_{2}\right) N\left((1 / 2,1 / 2), \pi^{\prime}, w_{2} w_{1} w_{2}\right)$ and

$$
\frac{L\left(1 / 2, \chi_{1} \pi_{2}\right) L\left(-1 / 2, \chi_{1} \pi_{2}\right)}{L\left(3 / 2, \chi_{1} \pi_{2}\right) \varepsilon\left(-1 / 2, \chi_{1} \pi_{2}\right) \varepsilon\left(1 / 2, \chi_{1} \pi_{2}\right)} N\left((1 / 2,1 / 2), \pi^{\prime}, w_{1} w_{2} w_{1} w_{2}\right) .
$$

Both residues are zero unless $L\left(1 / 2, \chi_{1} \pi_{2}\right) \neq 0$. Using the global functional equation

$$
L\left(-1 / 2, \chi_{1} \pi_{2}\right)=\varepsilon\left(-1 / 2, \chi_{1} \pi_{2}\right) L\left(3 / 2, \chi_{1} \pi_{2}\right)
$$

and $\varepsilon\left(1 / 2, \chi_{1} \pi_{2}\right)=1$ since $\chi_{1} \pi_{2}$ is selfcontragredient and $L\left(1 / 2, \chi_{1} \pi_{2}\right) \neq$ 0 . The latter residue simplifies to $L\left(1 / 2, \chi_{1} \pi_{2}\right) N\left((1 / 2,1 / 2), \pi^{\prime}, w_{1} w_{2} w_{1} w_{2}\right)$. Hence, decomposing the normalized intertwining operators as in Proposition 1.4 and using the discussion preceding the Theorem, the residue at $B$ equals

$$
L\left(1 / 2, \chi_{1} \pi_{2}\right)\left(1+\prod_{v} \eta_{v}\right) N\left((1 / 2,1 / 2), \pi^{\prime}, w_{2} w_{1} w_{2}\right) .
$$

Therefore, it is nonzero if $L\left(1 / 2, \chi_{1} \pi_{2}\right) \neq 0$ and the parity condition from the statement of the Theorem holds. Lemma 2.7 is satisfied and the residue equals the normalized intertwining operator $N\left((1 / 2,1 / 2), \pi^{\prime}, w_{2} w_{1} w_{2}\right)$.

It remains to prove the irreducibility of the image of that operator. Using Lemma 2.9 we prove that the image of the local intertwining operator $N\left((1 / 2,1 / 2), \pi_{v}^{\prime}, w_{2} w_{1} w_{2}\right)$ is irreducible at every place $v$. Since $N\left(w_{2} w_{1} w_{2}(1 / 2,1 / 2), w_{2} w_{1} w_{2}\left(\pi_{v}^{\prime}\right), w_{1}\right)=\eta_{v} I d_{v}$, that image is isomorphic to the image of $N\left((1 / 2,1 / 2), \pi_{v}^{\prime}, w_{1} w_{2} w_{1} w_{2}\right)$. Let $w=w_{1} w_{2} w_{1} w_{2}$ and $\underline{s}=(1 / 2,1 / 2)$. For a non-split place the image is irreducible since $\pi_{v}^{\prime}$ is a supercuspidal representation of $M_{0}^{\prime}\left(k_{v}\right)$ and $w$ is the longest element of the Weyl group.

At a split place $\chi_{1, v} \circ \operatorname{det}_{v}$ is the unique irreducible subrepresentation of

$$
\operatorname{Ind}_{\mathrm{GL}_{1}\left(k_{v}\right) \times \mathrm{GL}_{1}\left(k_{v}\right)}^{\mathrm{GL}_{2}\left(k_{v}\right)}\left(\chi_{1, v}|\cdot|^{-1 / 2} \otimes \chi_{1, v}|\cdot|^{1 / 2}\right)
$$

and $\pi_{2, v}$ is either tempered or a complementary series

$$
\pi_{2, v} \cong \operatorname{Ind}_{\mathrm{GL}_{1}\left(k_{v}\right) \times \mathrm{GL}_{1}\left(k_{v}\right)}^{\mathrm{GL}_{2}\left(k_{v}\right)}\left(\mu_{2, v}|\cdot|^{r} \otimes \mu_{2, v}|\cdot|^{-r}\right),
$$

where $0<r<1 / 2$ and $\mu_{2, v}$ is a unitary character of $k_{v}^{\times}$. Hence, $\pi_{v}$ is the unique irreducible subrepresentation of the induced representation from a tempered representation $\tau_{v}$ of a smaller parabolic subgroup $L\left(k_{v}\right)$. In the notation of Lemma 2.9, $L$ is one of the Levi factors $\mathrm{GL}_{1} \times \mathrm{GL}_{1} \times \mathrm{GL}_{2}$, $\mathrm{GL}_{1} \times \mathrm{GL}_{1} \times \mathrm{GL}_{1} \times \mathrm{GL}_{1}$ and

$$
\underline{s}+\underline{s}^{\prime}=\left\{\begin{array}{l}
(0,1,1 / 2), \\
(0,1,1 / 2+r, 1 / 2-r) .
\end{array}\right.
$$

In the notation of the proof of Proposition 1.12, let

$$
w^{\prime}=\left\{\begin{array}{l}
(1,2,3) \\
(1,2,3,4)
\end{array}\right.
$$


Then, $w^{-1}\left(\underline{s}+\underline{s}^{\prime}\right)$ is in the positive Weyl chamber and $N\left(w^{\prime-1}(\underline{s}+\right.$ $\left.\left.\underline{s}^{\prime}\right), w^{\prime-1}\left(\tau_{v}\right), w^{\prime}\right)$ is surjective onto $I\left(\underline{s}, \pi_{v}\right)$ because it can be decomposed into

$$
\begin{aligned}
\operatorname{Ind}\left(\chi_{1, v}|\cdot| \otimes \pi_{2, v} \nu^{1 / 2} \otimes \chi_{1, v}\right) & \rightarrow \operatorname{Ind}\left(\chi_{1, v}|\cdot| \otimes \chi_{1, v} \otimes \pi_{2, v} \nu^{1 / 2}\right) \\
& \rightarrow \operatorname{Ind}\left(\left(\chi_{1, v} \circ \operatorname{det}_{v}\right) \nu^{1 / 2} \otimes \pi_{2, v} \nu^{1 / 2}\right),
\end{aligned}
$$

where the first arrow is an isomorphism and the second one is surjective by the Langlands classification. Here Ind denotes the induction from the corresponding standard parabolic subgroup to $\mathrm{SO}_{8}\left(k_{v}\right)$. Let

$$
w^{\prime \prime}=\left\{\begin{array}{l}
(1)(2,3) \\
(1)(2,4)(3)
\end{array}\right.
$$

Then $w^{\prime \prime} w w^{\prime}$ is the longest element of the Weyl group of $\left(\mathrm{SO}_{8}, w^{-1}(L)\right)$ and $N\left(w\left(\underline{s}+\underline{s}^{\prime}\right), w\left(\tau_{v}\right), w^{\prime \prime}\right)$ is an isomorphism. Therefore, by Lemma 2.9 , the image of $N\left((1 / 2,1 / 2), \pi_{v}, w\right)$ is irreducible.

2.4. Case $C$. In this case both, $\pi_{1}$ and $\pi_{2}$, are residual automorphic representations of $\mathrm{GL}_{2}(\mathbb{A})$. This means that $\pi_{1}^{\prime}=\chi_{1} \circ \operatorname{det}^{\prime}$ and $\pi_{2}^{\prime}=\chi_{2} \circ \operatorname{det}^{\prime}$ are one-dimensional, and their global lifts are $\pi_{1}=\chi_{1} \circ \operatorname{det}$ and $\pi_{2}=\chi_{2} \circ$ det. Observe that by our definition the local lift of $\chi_{i, v} \circ \operatorname{det}_{v}^{\prime}$ at a non-split place $v$ is the Steinberg representation $S t_{\chi_{i, v}}$ of $\mathrm{GL}_{2}\left(k_{v}\right)$ which is not the local component at $v$ of the global lift.

The local normalizing factors, obtained in the previous Section, at the split places are given by (1.9) and in the maximal parabolic cases by (1.10) and (1.11). At the non-split places they are given using the local lifts by (1.16) and in the maximal parabolic cases by (1.18) and (1.17). As in case B, since the central characters of $\chi_{i, v} \circ \operatorname{det}_{v}$ and $S t_{\chi_{i, v}}$ are both equal to $\chi_{i, v}^{2}$, the local Hecke $\mathrm{L}$-functions and $\varepsilon$-factors appearing in the maximal parabolic case $\mathrm{GL}_{1}^{\prime} \subset G_{1}^{\prime}$ are at all places those of the central character $\chi_{i, v}^{2}$. Hence, in the global normalizing factor just the global Hecke L-functions and $\varepsilon$-factors of $\chi_{i}^{2}$ appear.

The local Rankin-Selberg L-function and $\varepsilon$-factor appearing in the local normalizing factor at a non-split place $v$ are $L\left(s, S t_{\chi_{1, v}} \times S t_{\chi_{2, v}^{-1}}\right)$ and $\varepsilon\left(s, S t_{\chi_{1, v}} \times S t_{\chi_{2, v}^{-1}}, \psi_{v}\right)$. By Theorem (3.1) and Sections 8 and 9 of [20], they are equal to

$$
\begin{aligned}
L\left(s, S t_{\chi_{1, v}} \times S t_{\chi_{2, v}^{-1}}\right)=L\left(s+1, \chi_{1, v} \chi_{2, v}^{-1}\right) L\left(s, \chi_{1, v} \chi_{2, v}^{-1}\right), \\
\varepsilon\left(s, S t_{\chi_{1, v}} \times S t_{\left.\chi_{2, v}^{-1}, \psi_{v}\right)}\right. \\
=\varepsilon\left(s+1, \chi_{1, v} \chi_{2, v}^{-1}, \psi_{v}\right) \varepsilon\left(s, \chi_{1, v} \chi_{2, v}^{-1}, \psi_{v}\right)^{2} \varepsilon\left(s-1, \chi_{1, v} \chi_{2, v}^{-1}, \psi_{v}\right) \\
\cdot \frac{L\left(1-s, \chi_{1, v}^{-1} \chi_{2, v}\right) L\left(-s, \chi_{1, v}^{-1} \chi_{2, v}\right)}{L\left(s-1, \chi_{1, v} \chi_{2, v}^{-1}\right) L\left(s, \chi_{1, v} \chi_{2, v}^{-1}\right)}
\end{aligned}
$$


Observe that the local L-functions appearing in the local normalizing factors at non-split places are not the same as in (1.10) at split places. Therefore, the global normalizing factor $r(s, \chi)$ for the maximal parabolic case $\mathrm{GL}_{1}^{\prime} \times \mathrm{GL}_{1}^{\prime} \subset$ $\mathrm{GL}_{2}^{\prime}$ is of the form

$$
\begin{aligned}
& \prod_{v \notin S_{D}} \frac{L\left(s, \chi_{v}\right) L\left(s-1, \chi_{v}\right)}{L\left(s+2, \chi_{v}\right) L\left(s+1, \chi_{v}\right) \varepsilon\left(s+1, \chi_{v}, \psi_{v}\right) \varepsilon\left(s, \chi_{v}, \psi_{v}\right)^{2} \varepsilon\left(s-1, \chi_{v}, \psi_{v}\right)} \\
& \cdot \prod_{v \in S_{D}}\left(\frac{L\left(s+1, \chi_{v}\right) L\left(s, \chi_{v}\right)}{L\left(s+2, \chi_{v}\right) L\left(s+1, \chi_{v}\right) \varepsilon\left(s+1, \chi_{v}, \psi_{v}\right) \varepsilon\left(s, \chi_{v}, \psi_{v}\right)^{2} \varepsilon\left(s-1, \chi_{v}, \psi_{v}\right)}\right. \\
& \left.\quad \cdot \frac{L\left(s, \chi_{v}\right) L\left(s-1, \chi_{v}\right)}{L\left(-s, \chi_{v}^{-1}\right) L\left(1-s, \chi_{v}^{-1}\right)}\right) \\
& =\frac{L(s, \chi) L(s-1, \chi)}{L(s+2, \chi) L(s+1, \chi) \varepsilon(s+1, \chi) \varepsilon(s, \chi)^{2} \varepsilon(s-1, \chi)} \\
& \quad \cdot \prod_{v \in S_{D}} \frac{L\left(s+1, \chi_{v}\right) L\left(s, \chi_{v}\right)}{L\left(1-s, \chi_{v}^{-1}\right) L\left(-s, \chi_{v}^{-1}\right)}
\end{aligned}
$$

where $s=s_{1} \pm s_{2}$ and $\chi=\chi_{1} \chi_{2}^{ \pm 1}$. As before, $S_{D}$ denotes the finite set of the non-archimedean places where $D$ is non-split.

\begin{tabular}{|c|c|}
\hline$w$ & $r\left(\underline{s}, \pi^{\prime}, w\right)$ \\
\hline \hline 1 & 1 \\
\hline \hline$w_{1}$ & $r\left(s_{1}-s_{2}, \chi_{1} \chi_{2}^{-1}\right)$ \\
\hline \hline$w_{2}$ & $\frac{L\left(2 s_{2}, \chi_{2}^{2}\right)}{L\left(1+2 s_{2}, \chi_{2}^{2}\right) \varepsilon\left(2 s_{2}, \chi_{2}^{2}\right)}$ \\
\hline \hline$w_{1} w_{2}$ & $\frac{L\left(2 s_{2}, \chi_{2}^{2}\right)}{L\left(1+2 s_{2}, \chi_{2}^{2}\right) \varepsilon\left(2 s_{2}, \chi_{2}^{2}\right)} r\left(s_{1}+s_{2}, \chi_{1} \chi_{2}\right)$ \\
\hline \hline$w_{2} w_{1}$ & $r\left(s_{1}-s_{2}, \chi_{1} \chi_{2}^{-1}\right) \frac{L\left(2 s_{1}, \chi_{1}^{2}\right)}{L\left(1+2 s_{1}, \chi_{1}^{2}\right) \varepsilon\left(2 s_{1}, \chi_{1}^{2}\right)}$ \\
\hline \hline$w_{1} w_{2} w_{1}$ & $r\left(s_{1}-s_{2}, \chi_{1} \chi_{2}^{-1}\right) \frac{L\left(2 s_{1}, \chi_{1}^{2}\right)}{L\left(1+2 s_{1}, \chi_{1}^{2}\right) \varepsilon\left(2 s_{1}, \chi_{1}^{2}\right)} r\left(s_{1}+s_{2}, \chi_{1} \chi_{2}\right)$ \\
\hline \hline$w_{2} w_{1} w_{2}$ & $\frac{L\left(2 s_{2}, \chi_{2}^{2}\right)}{L\left(1+2 s_{2}, \chi_{2}^{2}\right) \varepsilon\left(2 s_{2}, \chi_{2}^{2}\right)} r\left(s_{1}+s_{2}, \chi_{1} \chi_{2}\right) \frac{L\left(2 s_{1}, \chi_{1}^{2}\right)}{L\left(1+2 s_{1}, \chi_{1}^{2}\right) \varepsilon\left(2 s_{1}, \chi_{1}^{2}\right)}$ \\
\hline \hline$w_{1} w_{2} w_{1} w_{2}$ & $\frac{L\left(2 s_{2}, \chi_{2}^{2}\right)}{L\left(1+2 s_{2}, \chi_{2}^{2}\right) \varepsilon\left(2 s_{2}, \chi_{2}^{2}\right)} r\left(s_{1}+s_{2}, \chi_{1} \chi_{2}\right)$ \\
& $\frac{L\left(2 s_{1}, \chi_{1}^{2}\right)}{L\left(1+2 s_{1}, \chi_{1}^{2}\right) \varepsilon\left(2 s_{1}, \chi_{1}^{2}\right)} r\left(s_{1}-s_{2}, \chi_{1} \chi_{2}^{-1}\right)$ \\
\hline
\end{tabular}

TABle 7. Case C normalizing factors of $A\left(\underline{s}, \pi^{\prime}, w\right)$ for $w \in W\left(M_{0}^{\prime}\right)$

Therefore, the global normalizing factors in this case are given in Table 7. All the normalizing factors are expressed using the local and global Hecke L-functions. Their properties are given in Remark 2.11 and imply that the singular hyperplanes in this case are as shown in Figure 3.

While deforming the line of integration in (2.1) from $\underline{s}_{0}$ to the origin of $\mathfrak{a}_{M_{0}^{\prime}, \mathbb{C}}^{*}$ inside the positive Weyl chamber as in Figure 3 , we cross the singular 
hyperplanes. Taking the coordinate systems on those hyperplanes gives the points where the possible iterated poles of (2.2) occur. Also we consider the exceptional point $(3 / 2,-1 / 2)$ in Theorem 2.3 . There are five such points $C_{1}(5 / 2,1 / 2), C_{2}(2,0), C_{3}(3 / 2,-1 / 2), C_{4}(3 / 2,1 / 2)$ and $C_{5}(1 / 2,1 / 2)$ in Figure 3. Points $C_{1}, C_{2}$ and $C_{3}$ lie on the singular hyperplane $s_{1}-s_{2}=2$, while points $C_{4}$ and $C_{5}$ lie on $2 s_{2}=1$.

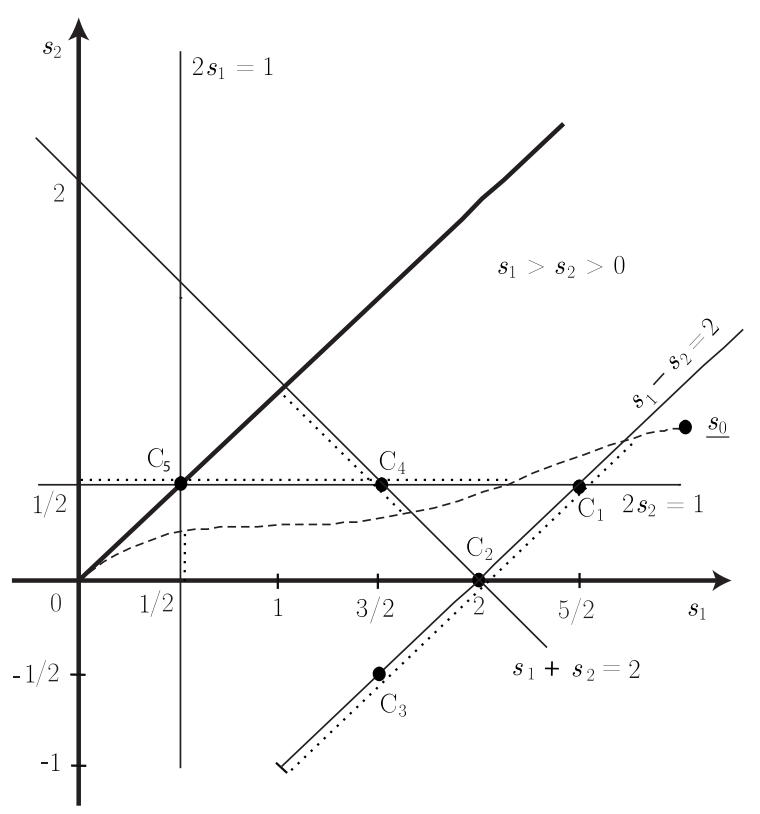

Figure 3. Case C singular hyperplanes

The coordinate system on $s_{1}-s_{2}=2$ is given by $s_{1}=z+1$ and $s_{2}=z-1$, where $z$ is the new variable. Points $C_{1}, C_{2}$ and $C_{3}$ have $z=3 / 2, z=1$ and $z=1 / 2$, respectively. For $2 s_{2}=1$ the coordinate system is given by $s_{1}=z$ and $s_{2}=1 / 2$, where $z$ is the new coordinate. Points $C_{4}$ and $C_{5}$ correspond to $z=3 / 2$ and $z=1 / 2$.

According to the four possible iterated poles, the case $\mathrm{C}$ contribution $L_{C}^{2}$ to the residual spectrum decomposes into

$$
L_{C}^{2} \cong L_{C_{1}}^{2} \oplus L_{C_{2}}^{2} \oplus L_{C_{3}}^{2} \oplus L_{C_{4}}^{2} \oplus L_{C_{5}}^{2},
$$

where, using the coordinate systems fixed above, $L_{C_{1}}^{2}, L_{C_{2}}^{2}, L_{C_{3}}^{2}, L_{C_{4}}^{2}$ and $L_{C_{5}}^{2}$ are obtained as the corresponding iterated residues of the Eisenstein series $E\left(\underline{s}, g ; f_{\underline{s}}, \pi^{\prime}\right)$ attached to the case $\mathrm{C}$ cuspidal automorphic representations $\pi^{\prime}$ of $M_{0}^{\prime}(\mathbb{A})$. In the following four Theorems those spaces are decomposed. 
THEOREM 2.26. The subspace $L_{C_{1}}^{2}$ of the residual spectrum of $G_{2}^{\prime}(\mathbb{A})$ decomposes into

$$
L_{C_{1}}^{2}=\oplus_{\pi^{\prime}} \mathcal{C}_{1}\left(\pi^{\prime}\right)
$$

where the sum is over all the one-dimensional cuspidal automorphic representations $\pi^{\prime} \cong\left(\chi \circ \operatorname{det}^{\prime}\right) \otimes\left(\chi \circ \operatorname{det}^{\prime}\right)$ of $M_{0}^{\prime}(\mathbb{A})$ such that $\chi^{2}$ is trivial.

$\mathcal{C}_{1}\left(\pi^{\prime}\right)$ is the irreducible space of automorphic forms spanned by the iterated residue

$$
\operatorname{Res}_{z=3 / 2} \operatorname{Res}_{s_{1}-s_{2}=2} E\left(\underline{s}, g ; f_{\underline{s}}, \pi^{\prime}\right)
$$

at $\underline{s}=(5 / 2,1 / 2)$ of the Eisenstein series attached to $\pi^{\prime}$. By (2.8), the constant term map induces an isomorphism of $\mathcal{C}_{1}\left(\pi^{\prime}\right)$ and the image of the normalized intertwining operator

$$
N\left((5 / 2,1 / 2), \pi^{\prime}, w_{1} w_{2} w_{1} w_{2}\right) .
$$

Proof. As in the previous cases, for the contribution at $C_{1}$ to the residual spectrum we first look at the pole along $s_{1}-s_{2}=2$. ¿From Table 7, the pole along the singular hyperplane $s_{1}-s_{2}=2$ occurs if and only if $\chi_{1}=\chi_{2}$, for the intertwining operators corresponding to the Weyl group elements $w_{1}$, $w_{2} w_{1}, w_{1} w_{2} w_{1}$ and $w_{1} w_{2} w_{1} w_{2}$ and it is simple. In the rest of the proof let $\chi=\chi_{1}=\chi_{2}$. Then Table 8 contains the residues along $s_{1}-s_{2}=2$, up to the nonzero constant

\begin{tabular}{|c|c|}
\hline$w$ & $\operatorname{Res}_{s_{1}-s_{2}=2} r\left(\underline{s}, \pi^{\prime}, w\right)$ \\
\hline$w_{1}$ & $\overline{1}$ \\
\hline$w_{2} w_{1}$ & $\frac{L\left(2 z+2, \chi^{2}\right)}{L\left(2 z+3, \chi^{2}\right) \varepsilon\left(2 z+2, \chi^{2}\right)}$ \\
\hline$w_{1} w_{2} w_{1}$ & $\begin{array}{c}\frac{L\left(2 z+2, \chi^{2}\right)}{L\left(2 z+3, \chi^{2}\right) \varepsilon\left(2 z+2, \chi^{2}\right)} \\
\frac{L\left(2 z-1, \chi^{2}\right) L\left(2 z, \chi^{2}\right)}{L\left(2 z+1, \chi^{2}\right) L\left(2 z+2, \chi^{2}\right) \varepsilon\left(2 z-1, \chi^{2}\right) \varepsilon\left(2 z, \chi^{2}\right)^{2} \varepsilon\left(2 z+1, \chi^{2}\right)} \\
\prod_{v \in S_{D}} \frac{L\left(2 z, \chi_{v}^{2}\right) L\left(2 z+1, \chi_{v}^{2}\right)}{L\left(-2 z, \chi_{v}^{-2}\right) L\left(1-2 z, \chi_{v}^{-2}\right)} \\
\end{array}$ \\
\hline$w_{1} w_{2} w_{1} w_{2}$ & $\begin{array}{c}L\left(2 z-2, \chi^{2}\right) \\
\frac{L\left(2 z+2, \chi^{2}\right)}{L\left(2 z-1, \chi^{2}\right) \varepsilon\left(2 z-2, \chi^{2}\right)} \frac{L\left(2 z+3, \chi^{2}\right) \varepsilon\left(2 z+2, \chi^{2}\right)}{L\left(2 z-1, \chi^{2}\right) L\left(2 z, \chi^{2}\right)} \\
\frac{L\left(2 z+1, \chi^{2}\right) L\left(2 z+2, \chi^{2}\right) \varepsilon\left(2 z-1, \chi^{2}\right) \varepsilon\left(2 z, \chi^{2}\right)^{2} \varepsilon\left(2 z+1, \chi^{2}\right)}{L\left(2 z, \chi_{v}^{2}\right) L\left(2 z+1, \chi_{v}^{2}\right)} \\
\prod_{v \in S_{D}} \frac{L\left(2 z, 2 z, \chi_{v}^{-2}\right) L\left(1-2 z, \chi_{v}^{-2}\right)}{L(-2)}\end{array}$ \\
\hline
\end{tabular}

$$
\frac{\operatorname{Res}_{s=1} L(s, \mathbf{1}) L(2, \mathbf{1})}{L(3, \mathbf{1}) L(4, \mathbf{1}) \varepsilon(1, \mathbf{1}) \varepsilon(2, \mathbf{1})^{2} \varepsilon(3, \mathbf{1})} \prod_{v \in S_{D}} \frac{L\left(2, \mathbf{1}_{v}\right) L\left(3, \mathbf{1}_{v}\right)}{L\left(-2, \mathbf{1}_{v}\right) L\left(-1, \mathbf{1}_{v}\right)},
$$

written in the new variable $z$.

TABLE 8. Residues along $s_{1}-s_{2}=2$ of case $\mathrm{C}$ normalizing factors

For the pole at $C_{1}$ we must look at the poles of the terms in Table 8 at $z=3 / 2$. The pole may occur only if $\chi$ is a quadratic character. Then, all the $\mathrm{L}$-functions in Table 8 are the Hecke $\mathrm{L}$-function of the trivial character and 
only the term corresponding to the Weyl group element $w_{1} w_{2} w_{1} w_{2}$ has the simple pole at $z=3 / 2$. Hence, the iterated residue at $C_{1}$, up to the nonzero constant, equals $N\left((5 / 2,1 / 2), \pi^{\prime}, w_{1} w_{2} w_{1} w_{2}\right)$. Since $w_{1} w_{2} w_{1} w_{2}(5 / 2,1 / 2)=$ $(-5 / 2,-1 / 2)$, Lemma 2.7 is satisfied.

It remains to prove the irreducibility of that image. Using Lemma 2.9 we prove the irreducibility of the image of $N\left((5 / 2,1 / 2), \pi_{v}^{\prime}, w_{1} w_{2} w_{1} w_{2}\right)$ at every place $v$. Let $w=w_{1} w_{2} w_{1} w_{2}$ and $\underline{s}=(5 / 2,1 / 2)$. At a non-split place the image is irreducible by the Langlands classification since $\pi_{v}^{\prime}$ is supercuspidal, $\underline{s}$ is in the positive Weyl chamber and $w$ is the longest element of the Weyl group.

For a split place, $\chi_{i, v} \circ \operatorname{det}_{v}$ is the unique irreducible subrepresentation of the induced representation

$$
\operatorname{Ind}_{\mathrm{GL}_{1}\left(k_{v}\right) \times \mathrm{GL}_{1}\left(k_{v}\right)}^{\mathrm{GL}_{2}\left(k_{v}\right)}\left(\mu_{i, v}|\cdot|^{-1 / 2} \otimes \mu_{i, v}|\cdot|^{1 / 2}\right) .
$$

Hence, $\pi_{v}$ is the unique irreducible subrepresentation induced from a tempered representation of $\mathrm{GL}_{1}\left(k_{v}\right) \times \mathrm{GL}_{1}\left(k_{v}\right) \times \mathrm{GL}_{1}\left(k_{v}\right) \times \mathrm{GL}_{1}\left(k_{v}\right)$ and, in the notation of Lemma $2.9, \underline{s}+\underline{s}^{\prime}=(2,3,0,1)$. Let $w^{\prime}$ of Lemma 2.9 be the Weyl group element corresponding, in the notation of the proof of Proposition 1.12, to $w^{\prime}=(1,2)(3,4)$. Then $w^{\prime-1}\left(\underline{s}+\underline{s}^{\prime}\right)$ is in the positive Weyl chamber and $N\left(w^{\prime-1}\left(\underline{s}+\underline{s}^{\prime}\right), w^{\prime-1}\left(\tau_{v}\right), w^{\prime}\right)$ is surjective onto $I\left(\underline{s}, \pi_{v}\right)$. Since $w w^{\prime}$ is the longest Weyl group element, let $w^{\prime \prime}=1$. Then, by Lemma 2.9, the image of $N\left((5 / 2,1 / 2), \pi_{v}, w\right)$ is irreducible.

Before decomposing $L_{C_{2}}^{2}$ we consider the induced representation

$$
\operatorname{Ind}_{\mathrm{GL}_{1}^{\prime}\left(k_{v}\right)}^{G_{1}^{\prime}\left(k_{v}\right)}\left(\chi_{v} \circ \operatorname{det}_{v}^{\prime}\right)
$$

where $\chi_{v}$ is a quadratic character. It is irreducible by Lemma 2.15 since the central character $\chi_{v}^{2}$ is trivial. Therefore, the normalized intertwining operator $N\left(0, \chi_{v} \circ \operatorname{det}_{v}^{\prime}, w_{2}\right)$ acts as $\pm I d_{v}$. Denote the sign by $\eta_{v}$.

THEOREM 2.27. The subspace $L_{C_{2}}^{2}$ of the residual spectrum of $G_{2}^{\prime}(\mathbb{A})$ decomposes into

$$
L_{C_{2}}^{2}=\oplus_{\pi^{\prime}} \mathcal{C}_{2}\left(\pi^{\prime}\right)
$$

where the sum is over all the one-dimensional cuspidal automorphic representations $\pi^{\prime} \cong\left(\chi \circ \operatorname{det}^{\prime}\right) \otimes\left(\chi \circ \operatorname{det}^{\prime}\right)$ of $M_{0}^{\prime}(\mathbb{A})$ such that $\chi$ is a quadratic character and the parity condition $\prod_{v} \eta_{v}=-1$ holds.

$\mathcal{C}_{2}\left(\pi^{\prime}\right)$ is the irreducible space of automorphic forms spanned by the iterated residue

$$
\operatorname{Res}_{z=1} \operatorname{Res}_{s_{1}-s_{2}=2} E\left(\underline{s}, g ; f_{\underline{s}}, \pi^{\prime}\right)
$$

at $\underline{s}=(2,0)$ of the Eisenstein series attached to $\pi^{\prime}$. By (2.8), the constant term map induces an isomorphism of $\mathcal{C}_{2}\left(\pi^{\prime}\right)$ and the image of the normalized intertwining operator

$$
N\left((2,0), \pi^{\prime}, w_{1} w_{2} w_{1}\right) .
$$


Proof. For the contribution at $C_{2}$ to the residual spectrum the pole along $s_{1}-s_{2}=2$ was already considered in the proof of the previous Theorem. It is simple, occurs only if $\chi=\chi_{1}=\chi_{2}$ and the residues are given in Table 8 .

Now, we must look at the pole at $z=1$ of the terms in the Table 8 . The pole may occur only if $\chi$ is a quadratic character. Then, the terms corresponding to $w_{1} w_{2} w_{1}$ and $w_{1} w_{2} w_{1} w_{2}$ have the simple pole and the residues, up to the nonzero constant

$$
\frac{L(4, \mathbf{1})}{L(5, \mathbf{1}) \varepsilon(4, \mathbf{1})} \frac{\operatorname{Res}_{s=1} L(s, \mathbf{1}) L(2, \mathbf{1})}{L(3, \mathbf{1}) L(4, \mathbf{1}) \varepsilon(1, \mathbf{1}) \varepsilon(2, \mathbf{1})^{2} \varepsilon(3, \mathbf{1})} \prod_{v \in S_{D}} \frac{L\left(2, \mathbf{1}_{v}\right) L\left(3, \mathbf{1}_{v}\right)}{L\left(-2, \mathbf{1}_{v}\right) L\left(-1, \mathbf{1}_{v}\right)},
$$

are equal to

$$
N\left((2,0),\left(\chi \circ \operatorname{det}^{\prime}\right) \otimes\left(\chi \circ \operatorname{det}^{\prime}\right), w_{1} w_{2} w_{1}\right)
$$

and

$$
-N\left((2,0),\left(\chi \circ \operatorname{det}^{\prime}\right) \otimes\left(\chi \circ \operatorname{det}^{\prime}\right), w_{1} w_{2} w_{1} w_{2}\right) .
$$

The sign in the latter comes from the fraction $L(2 z-2, \mathbf{1}) L(2 z-1, \mathbf{1})^{-1} \varepsilon(2 z-$ $2, \mathbf{1})^{-1}$ which is equal to -1 at $z=1$ by Lemma 2.8. According to Proposition 1.4, up to a nonzero constant, the residue at $C_{2}$ can be written acting on $\otimes_{v} f_{v}$ as

$$
\otimes_{v} N\left((2,0), \pi_{v}^{\prime}, w_{1} w_{2} w_{1}\right)\left[\otimes_{v} f_{v}-\otimes_{v} N\left((2,0), \pi_{v}^{\prime}, w_{2}\right) f_{v}\right] .
$$

But the normalized intertwining operator $N\left((2,0), \pi_{v}^{\prime}, w_{2}\right)$ is the $G_{1}^{\prime}\left(k_{v}\right)$ intertwining operator $N\left(0, \chi_{v} \circ \operatorname{det}_{v}^{\prime}, w_{2}\right)$. By the discussion preceding the statement of the Theorem it acts as $\eta_{v} I d_{v}$. Therefore the expression in the brackets is non-vanishing if $\prod_{v} \eta_{v}=-1$ and the residue is the normalized intertwining operator $N\left((2,0), \pi^{\prime}, w_{1} w_{2} w_{1}\right)$. It is square-integrable by Lemma 2.7 since $w_{1} w_{2} w_{1} w_{2}(2,0)=w_{1} w_{2} w_{1}(2,0)=(-2,0)$.

It remains to prove that the image of $N\left((2,0), \pi^{\prime}, w_{1} w_{2} w_{1}\right)$ is irreducible. Using Lemma 2.9 we prove that the image of the local normalized intertwining operators $N\left((2,0), \pi_{v}^{\prime}, w_{1} w_{2} w_{1}\right)$ is irreducible at every place $v$. At a non-split place $\chi_{v} \circ \operatorname{det}_{v}^{\prime}$ is supercuspidal and by the discussion preceding the statement of the Theorem the $G_{1}^{\prime}\left(k_{v}\right)$ induced representation $\operatorname{Ind}_{\mathrm{GL}_{1}^{\prime}\left(k_{v}\right)}^{G^{\prime}\left(k_{v}\right)}\left(\chi_{v} \circ \operatorname{det}_{v}^{\prime}\right)$ is irreducible and tempered. The Weyl group element $w_{1} w_{2} w_{1}$ is actually the longest Weyl group element for the $\mathrm{GL}_{1}^{\prime} \times G_{1}^{\prime} \subset G_{2}^{\prime}$ case and 2 is in the positive Weyl chamber. Hence, by the Langlands classification the image of $N\left((2,0), \pi_{v}^{\prime}, w_{1} w_{2} w_{1}\right)$ is irreducible.

At a split place $\pi_{v}$ is the unique irreducible subrepresentation of the induced representation

$$
\operatorname{Ind}_{T\left(k_{v}\right)}^{\mathrm{GL}_{2}\left(k_{v}\right) \times \mathrm{GL}_{2}\left(k_{v}\right)}\left(\left.\chi_{v}|\cdot|^{-1 / 2} \otimes \chi_{v}|\cdot|^{1 / 2} \otimes \chi_{v}|\cdot|\right|^{-1 / 2} \otimes \chi_{v}|\cdot|^{1 / 2}\right),
$$

where $T=\mathrm{GL}_{1} \times \mathrm{GL}_{1} \times \mathrm{GL}_{1} \times \mathrm{GL}_{1}$. Since $N\left((2,0), \pi_{v}, w_{2}\right)$ acts as $\eta_{v} I d_{v}$, it is an isomorphism and hence the image of $N\left((2,0), \pi_{v}^{\prime}, w_{1} w_{2} w_{1}\right)$ is the same as the image of $N\left((2,0), \pi_{v}^{\prime}, w_{1} w_{2} w_{1} w_{2}\right)$. Let $w=w_{1} w_{2} w_{1} w_{2}$ and $\underline{s}=$ $(2,0)$. In the notation of Lemma 2.9 , the Levi factor $L=T$, representation 
$\tau_{v}=\chi_{v} \otimes \chi_{v} \otimes \chi_{v} \otimes \chi_{v}$ is supercuspidal and $\underline{s}+\underline{s}^{\prime}=(3 / 2,5 / 2,-1 / 2,1 / 2)$. Let $w^{\prime}$ be the Weyl group element corresponding to the permutation $w^{\prime}=$ $(1,2)(3,4)$ and $w^{\prime \prime}=1$. Then $w^{\prime-1}\left(\underline{s}+\underline{s}^{\prime}\right)$ satisfies inequality (2.10) of Lemma $2.9, w^{\prime \prime} w w^{\prime}$ is the longest Weyl group element for $\left(\mathrm{SO}_{8}, T\right)$ and the image of $N\left(w^{\prime-1}\left(\underline{s}+\underline{s}^{\prime}\right), \tau_{v}, w^{\prime}\right)$ is precisely $I\left((2,0), \pi_{v}\right)$. Therefore, by Lemma 2.9 , the image of $N\left((2,0), \pi_{v}, w\right)$ is irreducible.

Before describing $L_{C_{3}}^{2}$ we need the composition series of the induced representation

$$
I=\operatorname{Ind}_{\mathrm{GL}_{1}^{\prime}(\mathbb{A})}^{G_{1}^{\prime}(\mathbb{A})}\left(\left(\chi \circ \operatorname{det}^{\prime}\right) \nu^{-1 / 2}\right) .
$$

At a split place let $T\left(k_{v}\right)$ be the maximal split torus of $\mathrm{SO}_{4}\left(k_{v}\right), P_{1}\left(k_{v}\right)$ and $P_{2}\left(k_{v}\right)$ the two maximal parabolic subgroups with Levi factors $L_{1}\left(k_{v}\right)$ and $L_{2}\left(k_{v}\right)$ isomorphic to $\mathrm{GL}_{2}\left(k_{v}\right)$, where $P_{1}\left(k_{v}\right)$ is the one with the property that the minimal parabolic subgroup defined over $k$ of $G_{1}^{\prime}\left(k_{v}\right)$ is an inner form of $P_{1}\left(k_{v}\right)$. Then, the local component at a split place $v$ of the induced representation above is the induced representation

$$
I_{v}=\operatorname{Ind}_{L_{1}\left(k_{v}\right)}^{\mathrm{SO}_{4}\left(k_{v}\right)}\left(\left(\chi_{v} \circ \operatorname{det}_{v}\right) \nu^{-1 / 2}\right) .
$$

The composition series for the local induced representations are considered in the following Lemma.

LEMMA 2.28. The local induced representation

$$
I_{v}=\operatorname{Ind}_{\mathrm{GL}_{1}^{\prime}\left(k_{v}\right)}^{G^{\prime}\left(k_{v}\right)}\left(\left(\chi_{v} \circ \operatorname{det}_{v}^{\prime}\right) \nu^{-1 / 2}\right)
$$

is at most of length two. It is irreducible if and only if $\chi_{v}^{2}$ is nontrivial.

At a split place $v$, if $\chi_{v}^{2}$ is trivial, then the subrepresentation is isomorphic to the Langlands quotient, denoted $X_{v}$, of the standard module

$$
\operatorname{Ind}_{T\left(k_{v}\right)}^{\mathrm{SO}_{4}\left(k_{v}\right)}\left(\chi_{v}|\cdot| \otimes \chi_{v}\right)
$$

while the quotient is isomorphic to the Langlands quotient, denoted $Y_{v}$, of the standard module

$$
\operatorname{Ind}_{L_{2}\left(k_{v}\right)}^{\mathrm{SO}_{4}\left(k_{v}\right)}\left(S t_{\chi_{v}} \nu^{1 / 2}\right)
$$

At a non-split place $v$, if $\chi_{v}^{2}$ is trivial, the subrepresentation is isomorphic to the Langlands quotient, denoted $X_{v}$, of

$$
\operatorname{Ind}_{\mathrm{GL}_{1}^{\prime}\left(k_{v}\right)}^{G_{1}^{\prime}\left(k_{v}\right)}\left(\left(\chi_{v} \circ \operatorname{det}_{v}^{\prime}\right) \nu^{1 / 2}\right)
$$

while the quotient, denoted $Y_{v}$, is square-integrable.

Proof. The first part of the Lemma follows from Lemma 2.15. For $v$ non-split, the composition series is obvious because $\chi_{v} \circ \operatorname{det}_{v}^{\prime}$ is supercuspidal.

If $v$ is split we look at the composition series of the induced representation

$$
V_{v}=\operatorname{Ind}_{T\left(k_{v}\right)}^{\mathrm{SO}_{4}\left(k_{v}\right)}\left(\chi_{v}|\cdot|^{-1} \otimes \chi_{v}\right) .
$$


By induction in stages, inducing to $L_{1}\left(k_{v}\right) \cong \mathrm{GL}_{2}\left(k_{v}\right)$ shows that both

$$
I_{v}=\operatorname{Ind}_{L_{1}\left(k_{v}\right)}^{\mathrm{SO}_{4}\left(k_{v}\right)}\left(\left(\chi_{v} \circ \operatorname{det}_{v}\right) \nu^{-1 / 2}\right)
$$

and

$$
J_{v}=\operatorname{Ind}_{L_{1}\left(k_{v}\right)}^{\mathrm{SO}_{4}\left(k_{v}\right)}\left(S t_{\chi_{v}} \nu^{-1 / 2}\right)
$$

are subquotients of $V_{v}$. Both representations $I_{v}$ and $J_{v}$ are of length two by Lemma 2.15. Thus $V_{v}$ is of length four. On the other hand, first inducing to $L_{2}\left(k_{v}\right) \cong \mathrm{GL}_{2}\left(k_{v}\right)$ shows that

$$
I_{v}^{\prime}=\operatorname{Ind}_{L_{2}\left(k_{v}\right)}^{\mathrm{SO}_{4}\left(k_{v}\right)}\left(\left(\chi_{v} \circ \operatorname{det}_{v}\right) \nu^{-1 / 2}\right)
$$

and

$$
J_{v}^{\prime}=\operatorname{Ind}_{L_{2}\left(k_{v}\right)}^{\mathrm{SO}_{4}\left(k_{v}\right)}\left(S t_{\chi_{v}} \nu^{-1 / 2}\right)
$$

are also subquotients of $V_{v}$, both of length two.

Observe that the exponents of $V_{v}$ satisfy the conditions for the open negative Weyl chamber. Thus, $V_{v}$ contains the unique irreducible subrepresentation which is the Langlands quotient $X_{v}$ of the first standard module in the Lemma. Since $I_{v}$ and $I_{v}^{\prime}$ are subrepresentations of $V_{v}$, they both contain $X_{v}$ as a subrepresentation.

It remains to determine the quotient of $I_{v}$. Observe that the exponents of $J_{v}$ and $J_{v}^{\prime}$ are in the negative open Weyl chamber. Hence, $J_{v}$ and $J_{v}^{\prime}$ have the corresponding Langlands quotients as subrepresentations. Then, the quotients of $J_{v}$ and $J_{v}^{\prime}$ are isomorphic since the length of $V_{v}$ is four. This also shows that the composition series of $V_{v}$ is multiplicity free. Now, the quotient of $I_{v}$ is precisely the irreducible subquotient of $V_{v}$ which does not appear as a subquotient of $J_{v}$ and that is the Langlands quotient $Y_{v}$ of $J_{v}^{\prime}$ as claimed.

Next, we study the poles at $s=-1 / 2$ of the local normalized intertwining operators $N\left(s, \chi \circ \operatorname{det}_{v}^{\prime}, w_{2}\right)$ acting on the induced representation $I_{v}$ decomposed in the previous Lemma. This is precisely the point excluded in Theorem 2.3 .

LEMMA 2.29. If $\chi_{v}^{2}$ is nontrivial, then the local normalized intertwining operator

$$
N\left(s, \chi_{v} \circ \operatorname{det}_{v}^{\prime}, w_{2}\right)
$$

is holomorphic and non-vanishing at $s=-1 / 2$. Moreover, it is an isomorphism.

If $\chi_{v}^{2}$ is trivial, then the local normalized intertwining operator

$$
N\left(s, \chi_{v} \circ \operatorname{det}_{v}^{\prime}, w_{2}\right)
$$

has a pole at $s=-1 / 2$. Moreover, the operator

$$
\tilde{N}\left(-1 / 2, \chi_{v} \circ \operatorname{det}_{v}^{\prime}, w_{2}\right)=\lim _{s \rightarrow-1 / 2}(s+1 / 2) N\left(s, \chi_{v} \circ \operatorname{det}_{v}^{\prime}, w_{2}\right)
$$


is holomorphic and non-vanishing. In the notation of the previous Lemma 2.28, its kernel is $X_{v}$ and its image is isomorphic to $Y_{v}$. Thus, $N\left(s, \chi_{v} \circ\right.$ $\left.\operatorname{det}_{v}^{\prime}, w_{2}\right)$ at $s=-1 / 2$ restricted to the subrepresentation $X_{v}$ of $I_{v}$ does not have a pole.

Proof. If $\chi_{v}^{2}$ is nontrivial, the claim on the holomorphy and nonvanishing is already proved in Lemma 1.12 for a split place and Lemma 1.22 for a non-split place. Then, the operator is an isomorphism since it acts on an irreducible representation.

First, let $v$ be a non-split place and $\chi_{v}^{2}$ trivial. The normalized intertwining operator $N\left(1 / 2, \chi_{v} \circ \operatorname{det}_{v}^{\prime}, w_{2}\right)$ is the operator of the Langlands classification. Hence, it is holomorphic, its kernel is isomorphic to $Y_{v}$ and its image is $X_{v}$. Since

$$
N\left(s, \chi_{v} \circ \operatorname{det}_{v}^{\prime}, w_{2}\right) N\left(-s, \chi_{v} \circ \operatorname{det}_{v}^{\prime}, w_{2}\right)=I d,
$$

the normalized operator $N\left(s, \chi_{v} \circ \operatorname{det}_{v}^{\prime}, w_{2}\right)$ has a pole at $s=-1 / 2$ because otherwise the composition would have nontrivial kernel. The pole is simple by Lemma 2.15 since the corresponding pole for $\mathrm{SL}_{2}\left(k_{v}\right)$ is simple. Furthermore, after cancelling the pole we have

$$
\widetilde{N}\left(-1 / 2, \chi_{v} \circ \operatorname{det}_{v}^{\prime}, w_{2}\right) N\left(1 / 2, \chi_{v} \circ \operatorname{det}_{v}^{\prime}, w_{2}\right)=0,
$$

and the holomorphic and non-vanishing operator $\tilde{N}\left(-1 / 2, \chi_{v} \circ \operatorname{det}_{v}^{\prime}, w_{2}\right)$ acts on length two representation. Thus, its kernel is the image of $N\left(1 / 2, \chi_{v} \circ\right.$ $\left.\operatorname{det}_{v}^{\prime}, w_{2}\right)$ which is $X_{v}$, while its image is the kernel of $N\left(1 / 2, \chi_{v} \circ \operatorname{det}_{v}^{\prime}, w_{2}\right)$ which is isomorphic to $Y_{v}$.

Finally, let $v$ be a split place and $\chi_{v}^{2}$ trivial. We use the notation of the proof of the previous Lemma 2.28. Consider the operator

$$
N\left((-1,0), \chi_{v} \otimes \chi_{v}, w_{2}\right),
$$

acting on the induced representation $V_{v}$. It is just the $L_{2}\left(k_{v}\right) \cong \mathrm{GL}_{2}\left(k_{v}\right)$ normalized operator. Hence, it has a simple pole at $(-1,0)$. After cancelling the pole, the kernel is $I_{v}^{\prime}$ and the image is isomorphic to $J_{v}^{\prime}$. Now $N\left(-1 / 2, \chi_{v} \circ\right.$ $\left.\operatorname{det}_{v}, w_{2}\right)$ is its restriction to $I_{v}$ and thus it has at most simple pole. After cancelling the pole, the operator $\widetilde{N}\left(-1 / 2, \chi_{v} \circ \operatorname{det}_{v}^{\prime}, w_{2}\right)$ has kernel isomorphic to the intersection of $I_{v}^{\prime}$ and $I_{v}$ which is $X_{v}$ and the image is isomorphic to the mutual subquotient of $J_{v}^{\prime}$ and $I_{v}$ which is $Y_{v}$.

The last claim follows directly from the fact that $X_{v}$ is the kernel of $\tilde{N}\left(-1 / 2, \chi \circ \operatorname{det}_{v}^{\prime}, w_{2}\right)$.

COROLlary 2.30. Let the notation be as above. If $\chi_{v}^{2}$ is nontrivial, then the image, which we denote $W_{v}^{\prime \prime}$, of the normalized intertwining operator

$$
N\left((3 / 2,-1 / 2),\left(\chi_{v} \circ \operatorname{det}_{v}^{\prime}\right) \otimes\left(\chi_{v} \circ \operatorname{det}_{v}^{\prime}\right), w_{1} w_{2} w_{1} w_{2}\right)
$$

is isomorphic to the Langlands quotient of the standard module

$$
\operatorname{Ind}_{T\left(k_{v}\right)}^{\mathrm{SO}_{8}\left(k_{v}\right)}\left(\chi_{v}|\cdot|^{2} \otimes \chi_{v}|\cdot| \otimes \chi_{v}|\cdot| \otimes \chi_{v}\right)
$$


at split places, and the Langlands quotient of the standard module

$$
\operatorname{Ind}_{\mathrm{GL}_{1}^{\prime}\left(k_{v}\right) \times \mathrm{GL}_{1}^{\prime}\left(k_{v}\right)}^{G_{2}^{\prime}\left(k_{v}\right)}\left(\left(\chi_{v} \circ \operatorname{det}_{v}^{\prime}\right) \nu^{3 / 2} \otimes\left(\chi_{v} \circ \operatorname{det}_{v}^{\prime}\right) \nu^{1 / 2}\right)
$$

at non-split places.

If $\chi_{v}^{2}$ is trivial, then the image of

$$
N\left((3 / 2,1 / 2),\left(\chi_{v} \circ \operatorname{det}_{v}^{\prime}\right) \otimes\left(\chi_{v} \circ \operatorname{det}_{v}^{\prime}\right), w_{1} w_{2} w_{1}\right) \tilde{N}\left(-1 / 2, \chi_{v} \circ \operatorname{det}_{v}^{\prime}, w_{2}\right),
$$

which we denote $W_{v}^{\prime}$ is isomorphic to the Langlands quotient of the standard module

$$
\operatorname{Ind}_{\mathrm{GL}_{1}\left(k_{v}\right) \times \mathrm{GL}_{1}\left(k_{v}\right) \times L_{2}\left(k_{v}\right)}^{\mathrm{SO}_{8}\left(k_{v}\right)}\left(\chi_{v}|\cdot|^{2} \otimes \chi_{v}|\cdot| \otimes S t_{\chi_{v}}\right)
$$

at split places, and the Langlands quotient of the standard module

$$
\operatorname{Ind}_{\mathrm{GL}_{1}^{\prime}\left(k_{v}\right) \times G_{1}^{\prime}\left(k_{v}\right)}^{G^{\prime}\left(k_{v}\right)}\left(\left(\chi_{v} \circ \operatorname{det}_{v}^{\prime}\right) \nu^{3 / 2} \otimes Y_{v}\right)
$$

at non-split places.

Furthermore, if $\chi_{v}^{2}$ is trivial, then the image of

$$
N\left((3 / 2,1 / 2),\left(\chi_{v} \circ \operatorname{det}_{v}^{\prime}\right) \otimes\left(\chi_{v} \circ \operatorname{det}_{v}^{\prime}\right), w_{1} w_{2} w_{1}\right),
$$

which we denote $W_{v}$, is non-trivial and contains $W_{v}^{\prime}$ as a subrepresentation.

Proof. The proof applies the idea of Lemma 2.9 with $w^{\prime \prime}=1$ and $w^{\prime}$ chosen appropriately. The choice of $w^{\prime}$ is based on Lemma 2.29.

Let $S$ be the finite set of places containing the set of archimedean places $S_{\infty}$, the set of non-split places $S_{D}$, and the set of all non-archimedean split places where $\chi_{v}$ is ramified. In other words, places $v \notin S$ are precisely nonarchimedean split unramified places. For $\chi^{2}$ nontrivial let $S_{1}$ be the (possibly empty) set of places where $\chi_{v}^{2}$ is trivial.

THEOREM 2.31. The subspace $L_{C_{3}}^{2}$ of the residual spectrum of $G_{2}^{\prime}(\mathbb{A})$ decomposes into

$$
L_{C_{3}}^{2}=\left(\oplus_{\pi^{\prime}} \mathcal{C}_{3}^{(1)}\left(\pi^{\prime}\right)\right) \oplus\left(\oplus_{\pi^{\prime}} \mathcal{C}_{3}^{(2)}\left(\pi^{\prime}\right)\right)
$$

The former sum is over all one-dimensional cuspidal automorphic representations $\pi^{\prime} \cong\left(\chi \circ \operatorname{det}^{\prime}\right) \otimes\left(\chi \circ \operatorname{det}^{\prime}\right)$ of $M_{0}^{\prime}(\mathbb{A})$ such that $\chi^{2}$ is trivial. The latter sum is over all one-dimensional cuspidal automorphic representations $\pi^{\prime} \cong\left(\chi \circ \operatorname{det}^{\prime}\right) \otimes\left(\chi \circ \operatorname{det}^{\prime}\right)$ of $M_{0}^{\prime}(\mathbb{A})$ such that $\chi^{2}$ is nontrivial, but there is at least one split place $v \notin S_{D}$ such that $\chi_{v}^{2}$ is trivial.

The spaces $\mathcal{C}_{3}^{(1)}\left(\pi^{\prime}\right)$ and $\mathcal{C}_{3}^{(2)}\left(\pi^{\prime}\right)$ are the spaces of automorphic forms generated by the residues

$$
\lim _{z \rightarrow 1 / 2}(z-1 / 2)^{n} \operatorname{Res}_{s_{1}-s_{2}=2} E\left(\underline{s}, g ; f_{\underline{s}}, \pi^{\prime}\right)
$$

at $\underline{s}=(3 / 2,-1 / 2)$ of the Eisenstein series attached to $\pi^{\prime}$, where $n$ is the order of the pole at $z=1 / 2$. 
If $\chi^{2}$ is trivial, then by (2.8) the constant term map induces an isomorphism of $\mathcal{C}_{3}^{(1)}\left(\pi^{\prime}\right)$ and

$$
\oplus_{V}\left[\left(\otimes_{v \in V} W_{v}^{\prime}\right) \otimes\left(\otimes_{v \notin V} W_{v}\right)\right],
$$

where the sum is over all finite sets of places $V$ such that $|V|=\left|S_{D}\right|$, and $W_{v}^{\prime}$, $W_{v}$ are the images of the intertwining operators defined in Corollary 2.30.

If $\chi^{2}$ is nontrivial, then by (2.8) the constant term map induces an isomorphism of $\mathcal{C}_{3}^{(2)}\left(\pi^{\prime}\right)$ and

$$
\oplus_{V}\left[\left(\otimes_{v \in V} W_{v}^{\prime}\right) \otimes\left(\otimes_{v \in S_{1} \backslash V} W_{v}\right) \otimes\left(\otimes_{v \notin S_{1}} W_{v}^{\prime \prime}\right)\right],
$$

where the sum is over all finite sets of places $V$ contained in the set $S_{1}$ of places where $\chi_{v}^{2}$ is trivial and such that $|V|=\left|S_{D} \cap S_{1}\right|+1$, and $W_{v}^{\prime}, W_{v}, W_{v}^{\prime \prime}$ are the images of the intertwining operators defined in Corollary 2.30.

Proof. The standard intertwining operators having nontrivial residues along $s_{1}-s_{2}=2$ correspond to the Weyl group elements $w_{1}, w_{2} w_{1}, w_{1} w_{2} w_{1}$ and $w_{1} w_{2} w_{1} w_{2}$. The first three normalized intertwining operators are holomorphic and non-vanishing at $C_{3}$ by Theorem 2.3. The residues along $s_{1}-s_{2}=2$ of their normalizing factors, given in Table 8 , are holomorphic at $C_{3}$ as well, i.e. at $z=1 / 2$ in the new variable $z$ on $s_{1}-s_{2}=2$ defined at the beginning of this Section 2.4. Recall that $z$ is given by $s_{1}=z+1$ and $s_{2}=z-1$. Hence, the possible pole at $z=1 / 2$ of the Eisenstein series coincides with the pole of the residue along $s_{1}-s_{2}=2$ of the standard intertwining operator

$$
\operatorname{Res}_{s_{1}-s_{2}=2} A\left(\left(s_{1}, s_{2}\right), \pi^{\prime}, w\right),
$$

where $w=w_{1} w_{2} w_{1} w_{2}, \pi^{\prime} \cong \chi \circ \operatorname{det}^{\prime} \otimes \chi \circ \operatorname{det}^{\prime}$ and $\chi$ is a unitary character of $\mathbb{A}^{\times} / k^{\times}$.

The difficulty in studying the analytic properties of that operator is due to the maximal parabolic case intertwining operator $A\left(z-1, \chi \circ \operatorname{det}^{\prime}, w_{2}\right)$ as we will see in a moment. Let us decompose

$$
\begin{aligned}
A\left(\left(s_{1}, s_{2}\right), \pi^{\prime}, w\right)= & A\left(\left(s_{1},-s_{2}\right), w_{2}\left(\pi^{\prime}\right), w_{1} w_{2} w_{1}\right) A\left(s_{2}, \chi \circ \operatorname{det}^{\prime}, w_{2}\right) \\
= & r\left(\left(s_{1},-s_{2}\right), w_{2}\left(\pi^{\prime}\right), w_{1} w_{2} w_{1}\right) \\
& N\left(\left(s_{1},-s_{2}\right), w_{2}\left(\pi^{\prime}\right), w_{1} w_{2} w_{1}\right) A\left(s_{2}, \chi \circ \operatorname{det}^{\prime}, w_{2}\right),
\end{aligned}
$$

according to the reduced decomposition of the Weyl group element. Along the singular hyperplane $s_{1}-s_{2}=2$ the intertwining operator corresponding to the Weyl group element $w_{1} w_{2} w_{1}$ has a simple pole coming from the normalizing factor. Therefore, up to the same non-zero constant as in the proof of Theorem 2.26, the residue $\operatorname{Res}_{s_{1}-s_{2}=2} A\left(\left(s_{1}, s_{2}\right), \pi^{\prime}, w\right)$ written in the new variable $z$ equals

$$
\frac{L\left(2 z-1, \chi^{2}\right) L\left(2 z, \chi^{2}\right)}{L\left(2 z+1, \chi^{2}\right) L\left(2 z+2, \chi^{2}\right) \varepsilon\left(2 z-1, \chi^{2}\right) \varepsilon\left(2 z, \chi^{2}\right)^{2} \varepsilon\left(2 z+1, \chi^{2}\right)}
$$




$$
\begin{gathered}
\left(\prod_{v \in S_{D}} \frac{L\left(2 z, \chi_{v}^{2}\right) L\left(2 z+1, \chi_{v}^{2}\right)}{L\left(-2 z, \chi_{v}^{-2}\right) L\left(1-2 z, \chi_{v}^{-2}\right)}\right) \frac{L\left(2 z+2, \chi^{2}\right)}{L\left(2 z+3, \chi^{2}\right) \varepsilon\left(2 z+2, \chi^{2}\right)} \\
\cdot N\left((z+1,1-z), w_{2}\left(\pi^{\prime}\right), w_{1} w_{2} w_{1}\right) A\left(z-1, \chi \circ \operatorname{det}^{\prime}, w_{2}\right) .
\end{gathered}
$$

At $z=1 / 2$ the normalized intertwining operator in (2.32) is holomorphic and non-vanishing. The scalar factor does not have a pole at $z=1 / 2$. Indeed, if $\chi^{2}$ is trivial, the simple poles of the two L-functions $L(2 z-1, \mathbf{1})$ and $L(2 z, \mathbf{1})$ are cancelled with the poles of the local L-functions $L\left(1-2 z, \mathbf{1}_{v}\right)$ for $v \in S_{D}$ since $\left|S_{D}\right| \geqslant 2$. Hence, if $\chi^{2}$ is trivial the scalar factor in (2.32) has a zero of order $\left|S_{D}\right|-2 \geqslant 0$ at $z=1 / 2$. If $\chi^{2}$ is nontrivial, then only the local L-functions $L\left(1-2 z, \chi_{v}^{2}\right)$ for $v \in S_{D}$ may have a pole at $z=1 / 2$. Indeed they have a simple pole if $\chi_{v}^{2}$ is trivial, i.e. $v \in S_{1}$. Hence, the order of a zero at $z=1 / 2$ of the scalar factor in (2.32) equals $\left|S_{1} \cap S_{D}\right| \geqslant 0$.

It remains to study the poles at $z=1 / 2$ of $A\left(z-1, \chi \circ \operatorname{det}^{\prime}, w_{2}\right)$. The order of the pole depends on the decomposable section $f_{z}=\left.f_{\underline{s}}\right|_{s_{1}-s_{2}=2}$ used to form the Eisenstein series. Let

$$
f_{z}=\left(\otimes_{v \in T} f_{z, v}\right) \otimes\left(\otimes_{v \notin T} f_{z, v}^{\circ}\right) \in I\left((z+1, z-1), \pi^{\prime}\right),
$$

where $T$ is a finite set of places containing $S$, and $f_{z, v}^{\circ}$ is the normalized unramified vector in $I\left((z+1, z-1), \pi^{\prime}\right)$ for $v \notin T$. Normalizing the standard intertwining operator acting on $f_{z}$ gives

$$
\begin{aligned}
A\left(z-1, \chi \circ \operatorname{det}^{\prime}, w_{2}\right) f_{z}= & \frac{L\left(2 z, \chi^{2}\right)}{L\left(1+2 z, \chi^{2}\right) \varepsilon\left(2 z, \chi^{2}\right)} \\
& {\left[\left(\otimes_{v \in T} N\left(z-1, \chi_{v} \circ \operatorname{det}_{v}^{\prime}, w_{2}\right) f_{z, v}\right) \otimes\left(\otimes_{v \notin T} \tilde{f}_{z, v}^{\circ}\right)\right], }
\end{aligned}
$$

where $\widetilde{f}_{z, v}^{\circ}$ is again normalized unramified vector in the appropriate induced representation. For $v \notin T$ this is justified by the fact that the unramified vector $f_{1 / 2, v}^{\circ}$ is in the subrepresentation $X_{v}$, and by Lemma 2.29 the normalized intertwining operator at $z=1 / 2$ does not have a pole there.

Consider first the case $\chi^{2}$ is trivial. Then, the global normalizing factor in (2.33) has a simple zero at $z=1 / 2$. The order of the pole at $z=1 / 2$ of (2.33) depends on the order of the pole of the local normalized intertwining operators

$$
N\left(z-1, \chi_{v} \circ \operatorname{det}_{v}^{\prime}, w_{2}\right) f_{z, v}
$$

for the places $v \in T$. Since $\chi_{v}^{2}$ is trivial for all $v$, Lemma 2.29 shows that the pole occurs if and only if $f_{z, v}$ is not in

$$
\operatorname{Ind}_{\mathrm{GL}_{1}^{\prime}\left(k_{v}\right) \times G_{1}^{\prime}\left(k_{v}\right)}^{G_{2}^{\prime}\left(k_{v}\right)}\left(\left(\chi_{v} \circ \operatorname{det}_{v}^{\prime}\right) \nu^{3 / 2} \otimes X_{v}\right) .
$$

Let $T_{0}\left(f_{z}\right)$ be the set of such places. Then, the order of the pole at $z=1 / 2$ of (2.33) equals $\left|T_{0}\left(f_{z}\right)\right|-1$. Looking back at the initial residue (2.32) acting on $f_{z}$, the order of the pole at $z=1 / 2$ equals $\left|T_{0}\left(f_{z}\right)\right|-\left|S_{D}\right|+1$. Observe 
that $T$ always contains $S_{D}$ and $S_{\infty}$ and hence $|T| \geqslant\left|S_{D}\right|+1$. Therefore, for any $T$ we can vary the local components $f_{z, v}$ at $v \in T$ in such a way that the number of places in $T_{0}\left(f_{z}\right)$ remains always greater than $\left|S_{D}\right|$ which assures that the Eisenstein series has a pole at $z=1 / 2$. In other words, for any finite set of places $V$ in $T$ such that $|V|=\left|S_{D}\right|$, we can choose any $f_{z, v}$ at $v \in T \backslash V$, while at every $v \in V$ we can choose only those $f_{z, v}$ which give a pole of the local intertwining operator, i.e. $f_{z, v}$ is not in the above induced representation involving $X_{v}$.

Since the Langlands square-integrability criterion of Lemma 2.7 is satisfied, cancelling the pole of the Eisenstein series, for a fixed $V \subset T$ such that $|V|=\left|S_{D}\right|$ we obtain the space of square-integrable automorphic forms which is isomorphic to

$$
\left[\left(\otimes_{v \in V} W_{v}^{\prime}\right) \otimes\left(\otimes_{v \in T \backslash V} W_{v}\right) \otimes\left(\otimes_{v \notin T} \widetilde{\widetilde{f}}_{1 / 2, v}^{0}\right)\right],
$$

where $W_{v}^{\prime}$ and $W_{v}$ are the images, described in Corollary 2.30, of the local intertwining operators after cancelling the pole where required, and $\widetilde{\widetilde{f}}_{1 / 2, v}$ is the normalized unramified vector of the appropriate induced representation. Recall that for $v \in T \backslash V$ we are allowed to vary $f_{z, v}$ without restriction giving $W_{v}$ as the image at those places.

Since the calculation holds for any finite set $T$ of places containing $S$ and all $V \subset T$ such that $|V|=\left|S_{D}\right|$ the space of automorphic forms obtained as residues in this case is precisely as claimed in the Theorem.

Next, we consider the case when $\chi^{2}$ is nontrivial. Then, the global normalizing factor in (2.33) is a non-zero constant at $z=1 / 2$. By Lemma 2.29, the local normalized intertwining operators in (2.33) do not have a pole at $z=1 / 2$ if $\chi_{v}^{2}$ is nontrivial, i.e. $v \notin S_{1}$. If $\chi_{v}^{2}$ is trivial, i.e. $v \in S_{1}$, the pole depends on the sections $f_{z, v}$. Namely, the simple pole occurs at $z=1 / 2$ if and only if $f_{1 / 2, v}$ is not in the subrepresentation

$$
\operatorname{Ind}_{\mathrm{GL}_{1}^{\prime}\left(k_{v}\right) \times G_{1}^{\prime}\left(k_{v}\right)}^{G_{2}^{\prime}\left(k_{v}\right)}\left(\left(\chi_{v} \circ \operatorname{det}_{v}^{\prime}\right) \nu^{3 / 2} \otimes X_{v}\right) .
$$

Let $T_{0}\left(f_{z}\right) \subset T \cap S_{1}$ be the set of such places. Now, looking back at (2.32), the order of the pole of the Eisenstein series attached to $f_{z}$ equals $\left|T_{0}\left(f_{z}\right)\right|-$ $\left|S_{1} \cap S_{D}\right|$. Thus if the set of places $T$ contains at least one place $v \in S_{1} \backslash S_{D}$, we can vary the local components in such a way that the Eisenstein series has a pole.

Proceeding as in the previous case, for any $V \subset T \cap S_{1}$ such that $|V|=$ $\left|S_{D} \cap S_{1}\right|+1$, cancelling the pole of the Eisenstein series we obtain the space of square-integrable automorphic forms isomorphic to

$$
\left[\left(\otimes_{v \in V} W_{v}^{\prime}\right) \otimes\left(\otimes_{v \in\left(T \cap S_{1}\right) \backslash V} W_{v}\right) \otimes\left(\otimes_{v \in T \backslash S_{1}} W_{v}^{\prime \prime}\right) \otimes\left(\otimes_{v \notin T} \widetilde{\widetilde{f}}_{1 / 2, v}^{\circ}\right)\right],
$$


where $W_{v}^{\prime}, W_{v}$ and $W_{v}^{\prime \prime}$ are the images, described in Corollary 2.30, of the local intertwining operators after cancelling the pole where required, and $\widetilde{\widetilde{f}}_{1 / 2, v}^{0}$ is the normalized unramified vector of the appropriate induced representation.

This holds for any finite set of places $T$ containing $S$ and at least one place of $S_{1} \backslash S_{D}$, and any $V \subset S_{1} \cap T$ such that $|V|=\left|S_{D} \cap S_{1}\right|+1$. Observe that the space is nontrivial if and only if such $T$ exists, i.e. $S_{1} \backslash S_{D}$ is nonempty. Varying $T$ shows that the obtained space of automorphic forms is as stated in the Theorem.

Corollary 2.34. The image $W_{v}$ (defined in Corollary 2.29) of the normalized intertwining operator

$$
N\left((3 / 2,1 / 2),\left(\chi_{v} \circ \operatorname{det}_{v}^{\prime}\right) \otimes\left(\chi_{v} \circ \operatorname{det}_{v}^{\prime}\right), w_{1} w_{2} w_{1}\right)
$$

where $\chi_{v}^{2}$ is trivial, is semisimple.

Proof. This is a consequence of the fact that $W_{v}$ is the local component at $v$ of an automorphic representation belonging to the residual spectrum.

THEOREM 2.35. The subspace $L_{C_{4}}^{2}$ of the residual spectrum of $G_{2}^{\prime}(\mathbb{A})$ is trivial, i.e. there is no contribution to the residual spectrum at point $C_{4}$.

Proof. For the contribution at $C_{4}$, first we look at the pole along the singular hyperplane $2 s_{2}=1$. From Table 7 , the pole along $2 s_{2}=1$ occurs if and only if $\chi_{2}^{2}$ is trivial for the intertwining operators corresponding to the Weyl group elements $w_{2}, w_{1} w_{2}, w_{2} w_{1} w_{2}$ and $w_{1} w_{2} w_{1} w_{2}$ and it is simple. The residues along $2 s_{2}=1$, up to the nonzero constant

$$
\operatorname{Res}_{s=1} L(s, \mathbf{1}) L(2, \mathbf{1})^{-1} \varepsilon(1, \mathbf{1})^{-1},
$$

written in the new variable $z$ are given in Table 9 .

The terms in Table 9 may have the simple pole at $z=3 / 2$ only if $\chi_{1} \chi_{2}$ is trivial i.e. $\chi=\chi_{1}=\chi_{2}$ is a quadratic character. But then, the terms corresponding to $w_{1} w_{2}$ and $w_{2} w_{1} w_{2}$ have the simple pole at $z=3 / 2$ and the term corresponding to $w_{1} w_{2} w_{1} w_{2}$ has the simple pole at $z=3 / 2$ only if $\left|S_{D}\right|=$ 2. Since $w_{1} w_{2}(3 / 2,1 / 2)=(-1 / 2,3 / 2)$ does not satisfy the Langlands square integrability criterion of Lemma 2.7 , the contribution from the corresponding term should be zero. In other words, if $f=\otimes_{v} f_{v}$ is an automorphic form in

$$
\operatorname{Ind}_{G L_{1}^{\prime}(\mathbb{A}) \times G L_{1}^{\prime}(\mathbb{A})}^{G_{2}^{\prime}(\mathbb{A})}\left(\left(\chi \circ \operatorname{det}^{\prime}\right) \nu^{3 / 2} \otimes\left(\chi \circ \operatorname{det}^{\prime}\right) \nu^{1 / 2}\right)
$$

then, in order to get a square-integrable residue, it should satisfy

$$
N\left((3 / 2,1 / 2), \pi^{\prime}, w_{1} w_{2}\right) f=0 .
$$




\begin{tabular}{|c|c|}
\hline$w$ & $\operatorname{Res}_{2 s_{2}=1} r\left(\underline{s}, \pi^{\prime}, w\right)$ \\
\hline$w_{2}$ & 1 \\
\hline$w_{1} w_{2}$ & $\begin{array}{c}L\left(z-1 / 2, \chi_{1} \chi_{2}\right) L\left(z+1 / 2, \chi_{1} \chi_{2}\right) \\
L\left(z+3 / 2, \chi_{1} \chi_{2}\right) L\left(z+5 / 2, \chi_{1} \chi_{2}\right) \varepsilon\left(z-1 / 2, \chi_{1} \chi_{2}\right) \varepsilon\left(z+1 / 2, \chi_{1} \chi_{2}\right)^{2} \varepsilon\left(z+3 / 2, \chi_{1} \chi_{2}\right) \\
\prod_{v \in S_{D} \frac{L\left(z+1 / 2, \chi_{1, v} \chi_{2, v}\right) L\left(z+3 / 2, \chi_{1, v} \chi_{2, v}\right)}{L\left(-z-1 / 2, \chi_{1, v}^{-1} \chi_{2, v}^{-1}\right) L\left(1 / 2-z, \chi_{1, v}^{-1} \chi_{2, v}^{-1}\right)}}^{L}\end{array}$ \\
\hline$w_{2} w_{1} w_{2}$ & $\begin{array}{c}L\left(z-1 / 2, \chi_{1} \chi_{2}\right) L\left(z+1 / 2, \chi_{1} \chi_{2}\right) \\
L\left(z+3 / 2, \chi_{1} \chi_{2}\right) L\left(z+5 / 2, \chi_{1} \chi_{2}\right) \varepsilon\left(z-1 / 2, \chi_{1} \chi_{2}\right) \varepsilon\left(z+1 / 2, \chi_{1} \chi_{2}\right)^{2} \varepsilon\left(z+3 / 2, \chi_{1} \chi_{2}\right) \\
\prod_{v \in S_{D}} \frac{L\left(z+1 / 2, \chi_{1, v} \chi_{2, v}\right) L\left(z+3 / 2, \chi_{1, v} \chi_{2, v}\right)}{L\left(-z-1 / 2, \chi_{1, v}^{-1} \chi_{2, v}^{-1}\right) L\left(1 / 2-z, \chi_{1, v}^{-1} \chi_{2, v}^{-1}\right)} \cdot \frac{L\left(2 z, \chi_{1}^{2}\right)}{L\left(2 z+1, \chi_{1}^{2}\right) \varepsilon\left(2 z, \chi_{1}^{2}\right)}\end{array}$ \\
\hline$w_{1} w_{2} w_{1} w_{2}$ & $\begin{array}{c}L\left(z+3 / 2, \chi_{1} \chi_{2}\right) L\left(z+5 / 2, \chi_{1} \chi_{2}\right) \varepsilon\left(z-1 / 2, \chi_{1} \chi_{2}\right) L\left(z+1 / 2, \chi_{1} \chi_{2}\right) \varepsilon\left(z+1 / 2, \chi_{1} \chi_{2}\right)^{2} \varepsilon\left(z+3 / 2, \chi_{1} \chi_{2}\right) \\
\prod_{v \in S_{D}} \frac{L\left(z+1 / 2, \chi_{1, v} \chi_{2, v}\right) L\left(z+3 / 2, \chi_{1, v} \chi_{2, v}\right)}{L\left(-z-1 / 2, \chi_{1, v}^{-1} \chi_{2, v}^{-1}\right) L\left(1 / 2-z, \chi_{1, v}^{-1} \chi_{2, v}^{-1}\right)} \cdot \frac{L\left(2 z, \chi_{1}^{2}\right)}{L\left(2 z+1, \chi_{1}^{2}\right) \varepsilon\left(2 z, \chi_{1}^{2}\right)} \\
\frac{L\left(z-3 / 2, \chi_{1} \chi_{2}\right) L\left(z-1 / 2, \chi_{1} \chi_{2}\right)}{L\left(z+1 / 2, \chi_{1} \chi_{2}\right) L\left(z+3 / 2, \chi_{1} \chi_{2}\right) \varepsilon\left(z-3 / 2, \chi_{1} \chi_{2}\right) \varepsilon\left(z-1 / 2, \chi_{1} \chi_{2}\right)^{2} \varepsilon\left(z+1 / 2, \chi_{1} \chi_{2}\right)} \\
\prod_{v \in S_{D} \frac{L\left(z-1 / 2, \chi_{1, v} \chi_{2, v}\right) L\left(z+1 / 2, \chi_{1, v} \chi_{2, v}\right)}{L\left(-z+1 / 2, \chi_{1, v}^{-1} \chi_{2, v}^{-1}\right) L\left(3 / 2-z, \chi_{1, v}^{-1} \chi_{2, v}^{-1}\right)}}\end{array}$ \\
\hline
\end{tabular}

TABLE 9. Residues along $2 s_{2}=1$ of case $\mathrm{C}$ normalizing factors

But then $L_{C_{4}}^{2}$ is trivial because the residues of the other two terms in Table 9 that may have the simple poles are up to a constants equal to

$$
\begin{aligned}
& N\left((3 / 2,1 / 2), \pi^{\prime}, w_{2} w_{1} w_{2}\right) f \\
& \quad=N\left((-1 / 2,3 / 2), \pi^{\prime}, w_{2}\right) N\left((3 / 2,1 / 2), \pi^{\prime}, w_{1} w_{2}\right) f=0, \\
& N\left((3 / 2,1 / 2), \pi^{\prime}, w_{1} w_{2} w_{1} w_{2}\right) f \\
& \quad=N\left((-1 / 2,3 / 2), \pi^{\prime}, w_{1} w_{2}\right) N\left((3 / 2,1 / 2), \pi^{\prime}, w_{1} w_{2}\right) f=0 .
\end{aligned}
$$

Before giving the decomposition of $L_{C_{5}}^{2}$ we introduce some notation. Consider the local normalized $\mathrm{GL}_{2}^{\prime}\left(k_{v}\right)$-intertwining operator $N\left(0,\left(\chi_{1, v} \circ \operatorname{det}_{v}^{\prime}\right) \otimes\right.$ $\left.\left(\chi_{2, v} \circ \operatorname{det}_{v}^{\prime}\right), w_{1}\right)$ acting on the induced representation

$$
\operatorname{Ind}_{\mathrm{GL}_{1}^{\prime}\left(k_{v}\right) \times \mathrm{GL}_{1}^{\prime}\left(k_{v}\right)}^{\mathrm{GL}^{\prime}\left(k_{v}\right)}\left(\left(\chi_{1, v} \circ \operatorname{det}_{v}^{\prime}\right) \otimes\left(\chi_{2, v} \circ \operatorname{det}_{v}^{\prime}\right)\right) .
$$

By Theorem 4.2 of [6] at split non-archimedean, Theorem B.2.d of [8] at nonsplit and results of [47] at archimedean places, this induced representation is irreducible. Hence, the normalized operator acts as $\pm I d_{v}$. Denote the sign by $\eta_{v}$. The following Lemma describes the image of the normalized intertwining operator $N\left((1 / 2,1 / 2),\left(\chi_{1, v} \circ \operatorname{det}_{v}^{\prime}\right) \otimes\left(\chi_{2, v} \circ \operatorname{det}_{v}^{\prime}\right), w_{2} w_{1} w_{2}\right)$, where $\chi_{1, v}$ and $\chi_{2, v}$ are quadratic characters of $k_{v}^{\times}$.

LEMMA 2.36. Let $\pi_{v}^{\prime} \cong\left(\chi_{1, v} \circ \operatorname{det}_{v}^{\prime}\right) \otimes\left(\chi_{2, v} \circ \operatorname{det}_{v}^{\prime}\right)$ be a representation of $M_{0}^{\prime}\left(k_{v}\right)$, where $\chi_{1, v}$ and $\chi_{2, v}$ are quadratic characters of $k_{v}^{\times}$. The image of the normalized intertwining operator $N\left((1 / 2,1 / 2), \pi_{v}^{\prime}, w_{2} w_{1} w_{2}\right)$ is irreducible at 
all non-split places and the split places where $\chi_{1, v}=\chi_{2, v}$, while it is the direct sum of two irreducible representations at the split places where $\chi_{1, v} \neq \chi_{2, v}$.

Proof. In the proof we apply Lemma 2.9 and use its notation. Since the normalized intertwining operator $N\left(w_{2} w_{1} w_{2}(1 / 2,1 / 2), w_{2} w_{1} w_{2}\left(\pi_{v}^{\prime}\right), w_{1}\right)=$ $\eta_{v} I d_{v}$, the image of $N\left((1 / 2,1 / 2), \pi_{v}^{\prime}, w_{2} w_{1} w_{2}\right)$ is isomorphic to the image of $N\left((1 / 2,1 / 2), \pi_{v}^{\prime}, w_{1} w_{2} w_{1} w_{2}\right)$. Let $w=w_{1} w_{2} w_{1} w_{2}$ and $\underline{s}=(1 / 2,1 / 2)$. For a non-split place the image is irreducible by the Langlands classification since $\pi_{v}^{\prime}$ is supercuspidal and $w$ is the longest Weyl group element.

Let $v$ be a split place. As in the proof of Theorem 2.26, $\pi_{v}$ is the unique irreducible subrepresentation of the induced representation from the torus $T \cong \mathrm{GL}_{1} \times \mathrm{GL}_{1} \times \mathrm{GL}_{1} \times \mathrm{GL}_{1}$ and, in the notation of Lemma 2.9, $L=T$ and

$$
\begin{gathered}
\underline{s}+\underline{s}^{\prime}=(0,1,0,1), \\
\tau_{v}=\chi_{1, v} \otimes \chi_{1, v} \otimes \chi_{2, v} \otimes \chi_{2, v} .
\end{gathered}
$$

For $w^{\prime}$ of Lemma 2.9 we take the Weyl group element corresponding, in the notation as in the proof of Proposition 1.12, to the permutation

$$
w^{\prime}=(1,4,3)(2) \text {. }
$$

Then

$$
\begin{gathered}
w^{\prime-1}\left(\underline{s}+\underline{s}^{\prime}\right)=(1,1,0,0), \\
w^{\prime-1}\left(\tau_{v}\right)=\chi_{2, v} \otimes \chi_{1, v} \otimes \chi_{1, v} \otimes \chi_{2, v} .
\end{gathered}
$$

Writing $w^{\prime}=(1)(2)(3,4) \circ(1,3,2)(4) \circ(1)(2,3)(4)$ shows that the normalized intertwining operator $N\left(w^{\prime-1}\left(\underline{s}+\underline{s}^{\prime}\right), w^{\prime-1}\left(\tau_{v}\right), w^{\prime}\right)$ is surjective onto $\operatorname{Ind}_{\mathrm{GL}_{2}\left(k_{v}\right) \times \mathrm{GL}_{2}\left(k_{v}\right)}^{\mathrm{SO}_{8}\left(k_{v}\right)}\left(\pi_{v}^{\prime}\right)$ since it decomposes into

$$
\begin{gathered}
\operatorname{Ind}_{T\left(k_{v}\right)}^{\mathrm{SO}_{8}\left(k_{v}\right)}\left(\chi_{2, v}|\cdot| \otimes \chi_{1, v}|\cdot| \otimes \chi_{1, v} \otimes \chi_{2, v}\right) \rightarrow \\
\operatorname{Ind}_{\mathrm{GL}_{1}\left(k_{v}\right) \times \mathrm{GL}_{2}\left(k_{v}\right) \times \mathrm{GL}_{1}\left(k_{v}\right)}^{\mathrm{SO}_{8}\left(k_{v}\right)}\left(\chi_{2, v}|\cdot| \otimes\left(\chi_{1, v} \circ \operatorname{det}_{v}\right) \nu^{1 / 2} \otimes \chi_{2, v}\right) \rightarrow \\
\operatorname{Ind}_{\mathrm{GL}_{2}\left(k_{v}\right) \times \mathrm{GL}_{1}\left(k_{v}\right) \times \mathrm{GL}_{1}\left(k_{v}\right)}^{\mathrm{SO}_{8}\left(k_{v}\right)}\left(\left(\chi_{1, v} \circ \operatorname{det}_{v}\right) \nu^{1 / 2} \otimes \chi_{2, v}|\cdot| \otimes \chi_{2, v}\right) \rightarrow \\
\operatorname{Ind}_{\mathrm{GL}_{2}\left(k_{v}\right) \times \mathrm{GL}_{2}\left(k_{v}\right)}^{\mathrm{SO}_{8}\left(k_{v}\right)}\left(\left(\chi_{1, v} \circ \operatorname{det}_{v}\right) \nu^{1 / 2} \otimes\left(\chi_{2, v} \circ \operatorname{det}_{v}\right) \nu^{1 / 2}\right),
\end{gathered}
$$

where the first and the third arrows are surjective and the second is an isomorphism.

Now, for $w^{\prime \prime}$ of Lemma 2.9 we take the Weyl group element such that $w^{\prime \prime} w w^{\prime}$ is the longest Weyl group element. Thus, $w^{\prime \prime}=(1,2,3)(4)$. We claim that the restriction of the normalized intertwining operator

$$
N\left(w\left(\underline{s}+\underline{s}^{\prime}\right), w\left(\tau_{v}\right), w^{\prime \prime}\right)
$$

acting on the induced representation

$$
\operatorname{Ind}_{T\left(k_{v}\right)}^{\mathrm{SO}_{8}\left(k_{v}\right)}\left(\chi_{1, v}^{-1}|\cdot| \otimes \chi_{1, v} \otimes \chi_{2, v}|\cdot|^{-1} \otimes \chi_{2, v}\right)
$$


to the subrepresentation

$$
\operatorname{Ind}_{\mathrm{GL}_{2}\left(k_{v}\right) \times \mathrm{GL}_{2}\left(k_{v}\right)}^{\mathrm{SO}_{8}\left(k_{v}\right)}\left(\left(\chi_{1, v} \circ \operatorname{det}_{v}\right) \nu^{-1 / 2} \otimes\left(\chi_{2, v} \circ \operatorname{det}_{v}\right) \nu^{-1 / 2}\right)
$$

is injective. In other words, its kernel does not intersect that subrepresentation. If $\chi_{1, v} \neq \chi_{2, v}$ it is an isomorphism since it is the product of two $\mathrm{GL}_{2}\left(k_{v}\right)$ normalized intertwining operators both acting on an irreducible induced representation. Otherwise, if $\chi_{1, v}=\chi_{2, v}=\chi_{v}$, then writing $w^{\prime \prime}=(1,2)(3)(4) \circ(1)(2,3)(4)$, it decomposes into

$$
\begin{gathered}
\operatorname{Ind}_{T\left(k_{v}\right)}^{\mathrm{SO}_{8}\left(k_{v}\right)}\left(\chi_{v}|\cdot|^{-1} \otimes \chi_{v} \otimes \chi_{v}|\cdot|^{-1} \otimes \chi_{v}\right) \rightarrow \\
\operatorname{Ind}_{T\left(k_{v}\right)}^{\mathrm{SO}_{8}\left(k_{v}\right)}\left(\chi_{v}|\cdot|^{-1} \otimes \chi_{v}|\cdot|^{-1} \otimes \chi_{v} \otimes \chi_{v}\right) \rightarrow \\
\operatorname{Ind}_{T\left(k_{v}\right)}^{\mathrm{SO}_{8}\left(k_{v}\right)}\left(\chi_{v}|\cdot|^{-1} \otimes \chi_{v}|\cdot|^{-1} \otimes \chi_{v} \otimes \chi_{v}\right),
\end{gathered}
$$

where the second arrow is just the isomorphism interchanging the first two characters. Hence, the kernel of $N\left(w\left(\underline{s}+\underline{s}^{\prime}\right), w\left(\tau_{v}\right), w^{\prime \prime}\right)$ is isomorphic to

$$
\operatorname{Ind}_{\mathrm{GL}_{1}\left(k_{v}\right) \times \mathrm{GL}_{2}\left(k_{v}\right) \times \mathrm{GL}_{1}\left(k_{v}\right)}^{\mathrm{SO}_{8}\left(k_{v}\right)}\left(\chi_{v}|\cdot|^{-1} \otimes S t_{\chi_{v}} \nu^{-1 / 2} \otimes \chi_{v}\right),
$$

where, at archimedean places, abusing the non-archimedean notation, we denote by $S t_{\chi_{v}}$ the unique irreducible subrepresentation of

$$
\operatorname{Ind}_{\mathrm{GL}_{1}\left(k_{v}\right) \times \mathrm{GL}_{1}\left(k_{v}\right)}^{\mathrm{GL}_{2}\left(k_{v}\right)}\left(\chi_{v}|\cdot|^{1 / 2} \otimes \chi_{v}|\cdot|^{-1 / 2}\right) .
$$

By the Langlands classification, the kernel contains the unique irreducible subrepresentation, namely the Langlands quotient. If the kernel intersected

$$
\operatorname{Ind}_{\mathrm{GL}_{2}\left(k_{v}\right) \times \mathrm{GL}_{2}\left(k_{v}\right)}^{\mathrm{SO}_{8}\left(k_{v}\right)}\left(\left(\chi_{1, v} \circ \operatorname{det}_{v}\right) \nu^{-1 / 2} \otimes\left(\chi_{2, v} \circ \operatorname{det}_{v}\right) \nu^{-1 / 2}\right),
$$

then the intersection would contain that Langlands quotient as a subrepresentation. However, such subrepresentation is isomorphic to a quotient of

$$
\operatorname{Ind}_{\mathrm{GL}_{2}\left(k_{v}\right) \times \mathrm{GL}_{2}\left(k_{v}\right)}^{\mathrm{SO}_{2}\left(k_{v}\right)}\left(\left(\chi_{1, v} \circ \operatorname{det}_{v}\right) \nu^{1 / 2} \otimes\left(\chi_{2, v} \circ \operatorname{det}_{v}\right) \nu^{1 / 2}\right)
$$

and, by the surjectivity of the normalized intertwining operator corresponding to $w^{\prime}$, it is a quotient of the induced representation

$$
\operatorname{Ind}_{T\left(k_{v}\right)}^{\mathrm{SO}_{8}\left(k_{v}\right)}\left(\chi_{v}|\cdot| \otimes \chi_{v}|\cdot| \otimes \chi_{v} \otimes \chi_{v}\right) .
$$

By the induction in stages, we first induce the last two characters to $\mathrm{SO}_{4}\left(k_{v}\right)$. This induced representation is irreducible, by Lemma 2.15 since $\chi_{v}$ is a quadratic character, and it is tempered. Denote it by $\sigma_{v}$. Then,

$$
\begin{aligned}
& \operatorname{Ind}_{T\left(k_{v}\right)}^{\mathrm{SO}_{8}\left(k_{v}\right)}\left(\chi_{v}|\cdot| \otimes \chi_{v}|\cdot| \otimes \chi_{v} \otimes \chi_{v}\right) \\
& \quad \cong \operatorname{Ind}_{\mathrm{GL}_{1}\left(k_{v}\right) \times \mathrm{GL}_{1}\left(k_{v}\right) \times \mathrm{SO}_{4}\left(k_{v}\right)}^{\mathrm{SO}_{8}\left(k_{v}\right)}\left(\chi_{v}|\cdot| \otimes \chi_{v}|\cdot| \otimes \sigma_{v}\right),
\end{aligned}
$$


and by the Langlands classification, this induced representation has the unique irreducible Langlands quotient which is not isomorphic to the Langlands quotient in the kernel. This shows our claim and thus injectivity for $\chi_{1, v}=\chi_{2, v}$.

Therefore, as in the proof of Lemma 2.9, by the surjectivity and injectivity proved above, the image of $N\left(w^{\prime-1}\left(\underline{s}+\underline{s}^{\prime}\right), w^{\prime-1}\left(\tau_{v}\right), w^{\prime \prime} w w^{\prime}\right)$ is isomorphic to the image of $N\left(\underline{s}, \pi_{v}^{\prime}, w\right)$. Recall that here $\underline{s}=(1 / 2,1 / 2)$. However, although $w^{\prime \prime} w w^{\prime}$ is the longest Weyl group element, $w^{\prime-1}\left(\underline{s}+\underline{s}^{\prime}\right)$ does not satisfy inequalities (2.10) of Lemma 2.9 and we can not apply the Langlands classification directly. Nevertheless, we can describe the image. By the induction in stages, as in the proof of the injectivity above,

$$
\begin{gathered}
\operatorname{Ind}_{T\left(k_{v}\right)}^{\mathrm{SO}_{8}\left(k_{v}\right)}\left(\chi_{2, v}|\cdot| \otimes \chi_{1, v}|\cdot| \otimes \chi_{1, v} \otimes \chi_{2, v}\right) \cong \\
\operatorname{Ind}_{\mathrm{GL}_{1}\left(k_{v}\right) \times \mathrm{GL}_{1}\left(k_{v}\right) \times \mathrm{SO}_{4}\left(k_{v}\right)}^{\mathrm{SO}_{8}\left(k_{v}\right)}\left(\chi_{2, v}|\cdot| \otimes \chi_{1, v}|\cdot| \otimes \operatorname{Ind}_{\mathrm{GL}_{1}\left(k_{v}\right) \times \mathrm{GL}_{1}\left(k_{v}\right)}^{\mathrm{SO}_{4}\left(k_{v}\right)}\left(\chi_{1, v} \otimes \chi_{2, v}\right)\right) .
\end{gathered}
$$

By Lemma 2.15, the $\mathrm{SO}_{4}\left(k_{v}\right)$ induced representation is irreducible and tempered if $\chi_{1, v}=\chi_{2, v}$ and it is the direct sum of two irreducible tempered representations if $\chi_{1, v} \neq \chi_{2, v}$. In any case, writing the longest Weyl group element as the product of the longest element for $\mathrm{GL}_{1} \times \mathrm{GL}_{1} \times \mathrm{SO}_{4} \subset \mathrm{SO}_{8}$ and the longest one for $\mathrm{GL}_{1} \times \mathrm{GL}_{1} \subset \mathrm{SO}_{4}$ allows the application of the Langlands classification for each tempered component of the $\mathrm{SO}_{4}\left(k_{v}\right)$ induced representation which proves the Lemma.

TheOREM 2.37. The subspace $L_{C_{5}}^{2}$ of the residual spectrum of $G_{2}^{\prime}(\mathbb{A})$ decomposes into

$$
L_{C_{5}}^{2}=\left(\oplus_{\pi^{\prime}} \mathcal{C}_{5}^{(1)}\left(\pi^{\prime}\right)\right) \oplus\left(\oplus_{\pi^{\prime}} \mathcal{C}_{5}^{(2)}\left(\pi^{\prime}\right)\right) .
$$

The former sum is over all the one-dimensional cuspidal automorphic representations $\pi^{\prime} \cong\left(\chi_{1} \circ \operatorname{det}^{\prime}\right) \otimes\left(\chi_{2} \circ \operatorname{det}^{\prime}\right)$ of $M_{0}^{\prime}(\mathbb{A})$ such that $\chi_{1} \neq \chi_{2}$ are both quadratic characters, $\chi_{1, v} \neq \chi_{2, v}$ for all $v \in S_{D}$ and the parity condition $\prod_{v} \eta_{v}=1$ holds. The latter sum is over all the one-dimensional cuspidal automorphic representations $\pi^{\prime} \cong\left(\chi_{1} \circ \operatorname{det}^{\prime}\right) \otimes\left(\chi_{2} \circ \operatorname{det}^{\prime}\right)$ of $M_{0}^{\prime}(\mathbb{A})$ such that $\chi_{1}=\chi_{2}$ is a quadratic character, the cardinality $\left|S_{D}\right|=2$ and the parity condition $\prod_{v} \eta_{v}=-1$ holds.

Both $\mathcal{C}_{5}^{(1)}\left(\pi^{\prime}\right)$ and $\mathcal{C}_{5}^{(2)}\left(\pi^{\prime}\right)$ are the spaces of automorphic forms spanned by the iterated residues

$$
\operatorname{Res}_{z=1 / 2} \operatorname{Res}_{2 s_{2}=1} E\left(\underline{s}, g ; f_{\underline{s}}, \pi^{\prime}\right)
$$

at $\underline{s}=(1 / 2,1 / 2)$ of the Eisenstein series attached to $\pi^{\prime}$. The space $\mathcal{C}_{5}^{(2)}\left(\pi^{\prime}\right)$ is irreducible and, by (2.8), the constant term map induces an isomorphism with the image of the normalized intertwining operator

$$
N\left((1 / 2,1 / 2), \pi_{v}^{\prime}, w_{2} w_{1} w_{2}\right) .
$$

By (2.8), the constant term map induces an isomorphism of $\mathcal{C}_{5}^{(1)}\left(\pi^{\prime}\right)$ and the sum of the irreducible spaces of the form $\otimes_{v} \Pi_{v}^{\prime}$, where $\Pi_{v}^{\prime}$ is one of at 
most two components of the image of the normalized intertwining operator $N\left((1 / 2,1 / 2), \pi_{v}^{\prime}, w_{2} w_{1} w_{2}\right)$ and it is the unramified one at almost all places.

Proof. For the contribution to the residual spectrum at $C_{5}$ we look first at the pole along the singular hyperplane $2 s_{2}=1$. It was already considered in the proof of the previous Theorem. The simple pole along $2 s_{2}=1$ occurs only if $\chi_{2}$ is a quadratic character. The residues written in the new variable $z$ are given in Table 9 . We must look at their poles at $z=1 / 2$.

First assume that $\chi_{1} \neq \chi_{2}$, i.e. $\chi_{1} \chi_{2}$ is nontrivial. Only the terms corresponding to $w_{2} w_{1} w_{2}$ and $w_{1} w_{2} w_{1} w_{2}$ may have the simple poles at $z=$ $1 / 2$ if $\chi_{1}$ is a quadratic character. The residues are, up to the nonzero constant

$\frac{\operatorname{Res}_{s=1} L(s, \mathbf{1})}{L(2, \mathbf{1}) \varepsilon(1, \mathbf{1})} \frac{L\left(0, \chi_{1} \chi_{2}\right) L\left(1, \chi_{1} \chi_{2}\right)}{L\left(2, \chi_{1} \chi_{2}\right) L\left(3, \chi_{1} \chi_{2}\right) \varepsilon\left(0, \chi_{1} \chi_{2}\right) \varepsilon\left(1, \chi_{1} \chi_{2}\right)^{2} \varepsilon\left(2, \chi_{1} \chi_{2}\right)}$.

$$
\cdot \prod_{v \in S_{D}} \frac{L\left(1, \chi_{1, v} \chi_{2, v}\right) L\left(2, \chi_{1, v} \chi_{2, v}\right)}{L\left(-1, \chi_{1, v} \chi_{2, v}\right)}
$$

equal to

$$
\prod_{v \in S_{D}} L\left(0, \chi_{1, v} \chi_{2, v}\right)^{-1} N\left((1 / 2,1 / 2), \pi^{\prime}, w_{2} w_{1} w_{2}\right)
$$

and

$$
\begin{gathered}
\frac{L\left(-1, \chi_{1} \chi_{2}\right) L\left(0, \chi_{1} \chi_{2}\right)}{L\left(1, \chi_{1} \chi_{2}\right) L\left(2, \chi_{1} \chi_{2}\right) \varepsilon\left(-1, \chi_{1} \chi_{2}\right) \varepsilon\left(0, \chi_{1} \chi_{2}\right)^{2} \varepsilon\left(1, \chi_{1} \chi_{2}\right)} . \\
\cdot \prod_{v \in S_{D}} L\left(0, \chi_{1, v} \chi_{2, v}\right)^{-1} N\left((1 / 2,1 / 2), \pi^{\prime}, w_{1} w_{2} w_{1} w_{2}\right) .
\end{gathered}
$$

By the global functional equation

$$
\begin{gathered}
L\left(-1, \chi_{1} \chi_{2}\right)=\varepsilon\left(-1, \chi_{1} \chi_{2}\right) L\left(2, \chi_{1} \chi_{2}\right), \\
L\left(0, \chi_{1} \chi_{2}\right)=\varepsilon\left(0, \chi_{1} \chi_{2}\right) L\left(1, \chi_{1} \chi_{2}\right), \\
\varepsilon\left(0, \chi_{1} \chi_{2}\right) \varepsilon\left(1, \chi_{1} \chi_{2}\right)=1,
\end{gathered}
$$

and hence the latter residue equals

$$
\prod_{v \in S_{D}} L\left(0, \chi_{1, v} \chi_{2, v}\right)^{-1} N\left((1 / 2,1 / 2), \pi^{\prime}, w_{1} w_{2} w_{1} w_{2}\right)
$$

Using the decomposition of the intertwining operators of Proposition 1.4, the residue at $C_{5}$ applied to $f=\otimes_{v} f_{v}$ can be written as

$$
\begin{aligned}
& \prod_{v \in S_{D}} L\left(0, \chi_{1, v} \chi_{2, v}\right)^{-1} \\
& \cdot \otimes_{v} N\left((1 / 2,1 / 2), \pi_{v}^{\prime}, w_{2} w_{1} w_{2}\right)\left(\otimes_{v} f_{v}+\otimes_{v} N\left((1 / 2,1 / 2), \pi_{v}^{\prime}, w_{1}\right) f_{v}\right) .
\end{aligned}
$$


The normalized intertwining operator $N\left((1 / 2,1 / 2), \pi_{v}^{\prime}, w_{1}\right)$ is actually the local intertwining operator considered before the statement of the Theorem. Hence, it acts as $\eta_{v} I d_{v}$ and the residue at $C_{5}$ equals

$$
\prod_{v \in S_{D}} L\left(0, \chi_{1, v} \chi_{2, v}\right)^{-1}\left(1+\prod_{v} \eta_{v}\right) N\left((1 / 2,1 / 2), \pi^{\prime}, w_{2} w_{1} w_{2}\right) .
$$

It is non-vanishing if and only if $\chi_{1, v} \neq \chi_{2, v}$ for all $v \in S_{D}$ and $\prod_{v} \eta_{v}=1$. Then, it equals the normalized operator $N\left((1 / 2,1 / 2), \pi^{\prime}, w_{2} w_{1} w_{2}\right)$. Since $w_{1} w_{2} w_{1} w_{2}(1 / 2,1 / 2)=w_{2} w_{1} w_{2}(1 / 2,1 / 2)=(-1 / 2,-1 / 2)$, Lemma 2.7 is satisfied.

Assume now that $\chi_{1}=\chi_{2}$, i.e. $\chi_{1} \chi_{2}$ is trivial. Then $\chi_{1}$ is a quadratic character. Consider the terms in Table 9. The term corresponding to $w_{1} w_{2}$ has the zero of order $\left|S_{D}\right|-2$ at $z=1 / 2$. Since $\left|S_{D}\right| \geqslant 2$ it does not have the pole at $z=1 / 2$. The terms corresponding to $w_{2} w_{1} w_{2}$ and $w_{1} w_{2} w_{1} w_{2}$ have the zero or pole of order $\left|S_{D}\right|-3$ at $z=1 / 2$. If $\left|S_{D}\right| \geqslant 4$ these two terms do not have the pole at $z=1 / 2$ and there is no contribution to the residual spectrum. Hence, let $\left|S_{D}\right|=2$. Then terms corresponding to $w_{2} w_{1} w_{2}$ and $w_{1} w_{2} w_{1} w_{2}$ have the simple pole at $z=1 / 2$. The residues are, up to the nonzero constant

$$
\begin{aligned}
& \frac{\operatorname{Res}_{s=1} L(s, \mathbf{1})}{L(2, \mathbf{1}) \varepsilon(1, \mathbf{1})} \frac{\operatorname{Res}_{s=0} L(s, \mathbf{1}) \operatorname{Res}_{s=1} L(s, \mathbf{1})}{L(2, \mathbf{1}) L(3, \mathbf{1}) \varepsilon(0, \mathbf{1}) \varepsilon(1, \mathbf{1})^{2} \varepsilon(2, \mathbf{1})} \\
& \cdot \prod_{v \in S_{D}} \frac{L\left(1, \mathbf{1}_{v}\right) L\left(2, \mathbf{1}_{v}\right)}{L\left(-1, \mathbf{1}_{v}\right) \operatorname{Res}_{s=0} L\left(s, \mathbf{1}_{v}\right)}
\end{aligned}
$$

equal to $N\left((1 / 2,1 / 2), \pi^{\prime}, w_{2} w_{1} w_{2}\right)$ and

$$
\begin{aligned}
& \left.\frac{L(-1, \mathbf{1})}{L(2, \mathbf{1}) \varepsilon(-1, \mathbf{1}) \varepsilon(0, \mathbf{1}) \varepsilon(1, \mathbf{1})} \cdot \frac{L(s, \mathbf{1})}{L(s+1, \mathbf{1}) \varepsilon(s, \mathbf{1})}\right|_{s=0} \\
& \left.\cdot \prod_{v \in S_{D}} \frac{L\left(s, \mathbf{1}_{v}\right)}{L\left(-s, \mathbf{1}_{v}\right)}\right|_{s=0} N\left((1 / 2,1 / 2), \pi^{\prime}, w_{1} w_{2} w_{1} w_{2}\right) .
\end{aligned}
$$

By the global functional equation we have $L(-1, \mathbf{1})=\varepsilon(-1, \mathbf{1}) L(2, \mathbf{1})$ and $\varepsilon(0, \mathbf{1}) \varepsilon(1, \mathbf{1})=1$ and Lemma 2.8

$$
\left.L(s, \mathbf{1}) L(s+1, \mathbf{1})^{-1} \varepsilon(s, \mathbf{1})^{-1}\right|_{s=0}=-1
$$

and

$$
\left.\prod_{v \in S_{D}} L\left(s, \mathbf{1}_{v}\right) L\left(-s, \mathbf{1}_{v}\right)^{-1}\right|_{s=0}=(-1)^{\left|S_{D}\right|}=1
$$

Hence, the latter residue is equal to $-N\left((1 / 2,1 / 2), \pi^{\prime}, w_{1} w_{2} w_{1} w_{2}\right)$. As in the previous case, decomposing the intertwining operators and the discussion 
preceding the statement of the Theorem give for the residue

$$
\left(1-\prod_{v} \eta_{v}\right) N\left((1 / 2,1 / 2), \pi^{\prime}, w_{2} w_{1} w_{2}\right)
$$

Therefore, the residue is nonzero if $\prod_{v} \eta_{v}=-1$ as claimed. Lemma 2.7 is satisfied as above and the residue equals the normalized intertwining operator $N\left((1 / 2,1 / 2), \pi^{\prime}, w_{2} w_{1} w_{2}\right)$.

In both cases, the image of the normalized intertwining operator

$$
N\left((1 / 2,1 / 2), \pi^{\prime}, w_{2} w_{1} w_{2}\right)
$$

is described locally at every place in Lemma 2.36, thus finishing the proof.

\section{REFERENCES}

[1] J. Arthur, Intertwining Operators and Residues I. Weighted Characters, J. Funct. Anal. 84 (1989), 19-84.

[2] J. Arthur, Unipotent automorphic representations: conjectures, in: Orbites unipotentes et représentations II, Astérisque 171-172 (1989), 13-71.

[3] J. Arthur, Unipotent automorphic representations: global motivation, in: L. Clozel, J.S. Milne, eds., Automorphic Forms, Shimura Varieties, and $L$-functions I., Academic Press, 1990, 1-75.

[4] J. Arthur, An Introduction to the trace formula, in: Harmonic analysis, the trace formula and Shimura varieties, 2005, 1-263.

[5] J. Arthur and L. Clozel, Simple algebras, base change, and the advanced theory of the trace formula, Princeton Univiversity Press, Princeton, 1989.

[6] I. N. Bernstein and A. V. Zelevinsky, Induced representations of reductive $\mathfrak{p}$-adic Groups I, Ann. Sci. École Norm. Sup. (4) 10 (1977), 441-472.

[7] W. Casselman and F. Shahidi, On irreducibility of standard modules for generic representations, Ann. Sci. École Norm. Sup. (4) 31 (1998), 561-589.

[8] P. Deligne, D. Kazhdan and M. F. Vignéras, Représentations des algèbres centrales simples p-adiques, Représentations des Groupes Réductifs sur un Corps Local, Herman, Paris, 1984, 33-117.

[9] S. Gelbart and H. Jacquet, A relation between automorphic representations of $G L(2)$ and $G L(3)$, Ann. Sci. Éc. Norm. Sup. (4) 11 (1978), 471-542.

[10] S. Gelbart and H. Jacquet, Forms of GL(2) from the analytic point of view, in: Automorphic forms, representations and $L$-functions Proc. Sympos. Pure Math. 33, part 1, 1979, 213-251.

[11] N. Grbac, Correspondence between the residual spectra of rank two split classical groups and their inner forms, in: Functional analysis IX (Dubrovnik, 2005), 44-57, Various Publ. Ser. 48, Univ. Aarhus, Aarhus, 2007.

[12] N. Grbac, On a relation between residual spectra of split classical groups and their inner forms, Canad. J. Math., to appear.

[13] N. Grbac, The residual spectrum of an inner form of $\mathrm{Sp}_{8}$ supported in the minimal parabolic subgroup, Trans. Amer. Math. Soc., to appear.

[14] N. Grbac, The residual spectrum of $\mathrm{GL}_{n}$ over a division algebra, an appendix to: A. I. Badulescu, Global Jacquet-Langlands correspondence, multiplicity one and classification of automorphic representations, Invent. Math., 172 (2008), 383-438. 
[15] N. Grbac, Arthur parameters for the residual spectrum of certain low rank Hermitian quaternionic classical groups, in preparation.

[16] M. Hanzer, $R$ groups for quaternionic Hermitian groups, Glas. Mat. Ser. III 39(59) (2004), 31-48.

[17] M. Hanzer, The unitary dual of the Hermitian quaternionic group of split rank 2, Pacific J. Math 226 (2006), 353-388.

[18] H. Jacquet, Principal L-functions of the linear group, Proc. Sympos. Pure Math. 33, part 2, 1979, 63-86.

[19] H. Jacquet, On the residual spectrum of $G L(N)$, Lie Group Representations II, Lecture Notes in Math. 1041, Springer, Berlin, 1984, 185-208.

[20] H. Jacquet, I. I. Piatetskii-Shapiro and J. A. Shalika, Rankin-Selberg convolutions, Amer. J. Math. 105 (1983), 367-464.

[21] C. D. Keys and F. Shahidi, Artin L-functions and normalization of intertwining operators, Ann. Sci. École Norm. Sup. (4) 21 (1988), 67-89.

[22] H. H. Kim, The residual spectrum of $\mathrm{Sp}_{4}$, Compositio Math. 99 (1995), 129-151.

[23] H. H. Kim, The residual spectrum of $G_{2}$, Canad. J. Math. 48 (1996), 1245-1272.

[24] H. H. Kim, Langlands-Shahidi method and poles of automorphic L-functions: application to exterior square L-functions, Canad. J. Math. 51 (1999), 835-849.

[25] H. H. Kim, Langlands-Shahidi method and poles of automorphic L-functions II, Israel J. Math. 117 (2000), 261-284.

[26] H. H. Kim, Residual spectrum of odd orthogonal groups, Internat. Math. Res. Notices 17 (2001), 873-906.

[27] T. Kon-No, The residual spectrum of $U(2,2)$, Trans. Amer. Math. Soc. 350 (1998), 1285-1358.

[28] B. Kostant, On Whittaker vectors and representation theory, Invent. Math. 48 (1978), 101-184.

[29] J.-P. Labesse, R. P. Langlands, L-indistinguishability for $S L(2)$, Canad. J. Math. 31 (1979), 726-785.

[30] R. P. Langlands, On the functional equations satisfied by Eisenstein series, Lecture Notes in Math. 544, Springer-Verlag, 1976.

[31] R. P. Langlands, On the notion of an automorphic representation, A supplement to the paper: A. Borel and H. Jacquet, Automorphic forms and automorphic representations, Proc. Sympos. Pure Math. 33, part 1 (1979), 203-207.

[32] E. Lapid, G. Muić and M. Tadić, On the generic unitary dual of quasisplit classical groups, Int. Math. Res. Not. 26 (2004), 1335-1354.

[33] C. Møglin, Orbites unipotentes et spectre discret non ramifie, Compositio Math. 77 (1991), 1-54.

[34] C. Møglin, Représentations unipotentes et formes automorphes de carré intégrable, Forum Math. 6 (1994), 651-744.

[35] C. Møglin, Conjectures sur le spectre residuel, J. Math. Soc. Japan 53 (2001), 395427.

[36] C. Mœglin, Formes automorphes de carré intégrable non cuspidales, preprint.

[37] C. Møglin and J.-L. Waldspurger, Le spectre résiduel de $G L(n)$, Ann. Sci. École Norm. Sup. (4) 22 (1989), 605-674.

[38] C. Møglin and J.-L. Waldspurger, Spectral decomposition and Eisenstein series: Une paraphrase de l'Ecriture, Cambridge Tracts in Math. 113, Cambridge University Press, Cambridge, 1995.

[39] G. Muić, Some results on square integrable representations; irreducibility of standard representations, Internat. Math. Res. Notices 14 (1998), 705-726.

[40] G. Muić, A proof of Casselman-Shahidi's conjecture for quasi-split classical groups, Canad. Math. Bull. 44 (2001), 298-312. 
[41] G. Muić, On certain classes of unitary representations for split classical groups, Canad. J. Math. 59 (2007), 148-185.

[42] G. Muić and G. Savin, Complementary series for Hermitian quaternionic groups, Canad. Math. Bull. 43 (2000), 90-99.

[43] F. Shahidi, On certain L-functions, Amer. J. Math. 103 (1981), 297-355.

[44] F. Shahidi, Local coefficients as Artin factors for real groups, Duke Math. J. 52 (1985), 973-1007.

[45] F. Shahidi, A proof of Langlands' conjecture on Plancherel measures; complementary series of $\mathfrak{p}$-adic groups, Ann. of Math. 132 (1990), 273-330.

[46] F. Shahidi, Twisted endoscopy and reducibility of induced representations for p-adic groups, Duke Math. J. 66 (1992), 1-41.

[47] B. Speh, The unitary dual of $G l(3, \mathbb{R})$ and $G l(4, \mathbb{R})$, Math. Ann. 258 (1981/1982), $113-133$.

[48] M. Tadić, Induced representations of $G L(n, A)$ for $p$-adic division algebras $A, \quad J$. Reine Angew. Math. 405 (1990), 48-77.

[49] M. Tadić, Notes on representations of non-archimedean $S L(n)$, Pacific J. Math. 152 (1992), 375-396.

[50] D. Vogan, Gelfand-Kirillov dimension for Harish-Chandra modules, Invent. Math. 48 (1978), 75-98.

[51] N. R. Wallach, Real reductive groups I, Pure and Applied Math. 132, Academic Press, Inc., Boston, 1988

[52] G. Warner, Harmonic analysis on semi-simple Lie groups I, Die Grundlehren der mathematischen Wissenschaften 188, Springer-Verlag, New York-Heidelberg, 1972.

[53] A. V. Zelevinsky, Induced representations of reductive $\mathfrak{p}$-adic groups II. On irreducible representations of $G L(n)$, Ann. Sci. École Norm. Sup. (4) 13 (1980), 165-210.

[54] Y. Zhang, The holomorphy and nonvanishing of normalized local intertwining operators, Pacific J. Math. 180 (1997), 385-398.

[55] S. Žampera, The residual spectrum of the group of type $G_{2}$, J. Math. Pures Appl. 76 (1997), 805-835.

\section{N. Grbac}

Department of Mathematics

University of Rijeka

Omladinska 14

51000 Rijeka

Croatia

E-mail: neven.grbac@math.uniri.hr

Received: 27.4.2008.

Revised: 17.5.2008. 